ROBERTO DE OLIVEIRA POSSIDENTE

\title{
CANCELAMENTO DE ECO ACÚSTICO NÃO-LINEAR COM O USO DE UM MODELO FÍSICO DO ALTO-FALANTE
}

Dissertação apresentada à Escola Politécnica da Universidade de São Paulo para obtenção do Título de Mestre em Ciências. 


\section{ROBERTO DE OLIVEIRA POSSIDENTE}

\section{CANCELAMENTO DE ECO ACÜSTICO NÃO-LINEAR COM O USO DE UM MODELO FÍSICO DO ALTO-FALANTE}

Dissertação apresentada à Escola Politécnica da Universidade de São Paulo para obtenção do Título de Mestre em Ciências.

Área de Concentração:

Engenharia Elétrica - Sistemas Eletrônicos

Orientador:

Prof. Dr. Phillip Mark Seymour Burt 
Autorizo a reprodução e divulgação total ou parcial deste trabalho, por qualquer meio convencional ou eletrônico, para fins de estudo e pesquisa, desde que citada a fonte.

Este exemplar foi revisado e corrigido em relação à versão original, sob responsabilidade única do autor e com a anuência de seu orientador.

São Paulo, de de

Assinatura do autor:

Assinatura do orientador:

\section{Catalogação-na-publicação}

Possidente, Roberto de Oliveira

Cancelamento de eco acústico não-linear com o uso de um modelo físico do alto-falante. / R. O. Possidente -- versão corr. -- São Paulo, 2019. $170 \mathrm{p}$.

Dissertação (Mestrado) - Escola Politécnica da Universidade de São Paulo. Departamento de Engenharia de Telecomunicações e Controle.

1.Processamento digital de sinais 2.Filtros elétricos adaptativos 3.Sistemas não-lineares I.Universidade de São Paulo. Escola Politécnica. Departamento de Engenharia de Telecomunicações e Controle II.t. 
Dedico este trabalho à minha família. Sem vocês nada disso teria sentido! 


\section{AGRADECIMENTOS}

Desejo expressar os meus sinceros agradecimentos:

Ao meu orientador, Prof. Dr. Phillip M. S. Burt, pela orientação, apoio e paciência ao longo deste trabalho.

À minha mãe, Marlene, pelos ensinamentos passados por toda minha vida, além de ter sido exemplo de perseverança e força de vontade durante essa longa jornada.

Em especial, à minha esposa, Tatiane, pelo amor, apoio e compreensão!

Aos meus filhos, Matheus e Isabela, por me darem motivação e comprovação de que tudo isso vale a pena! 


\section{RESUMO}

Em sistemas de comunicação viva-voz, o eco acústico origina-se da reprodução do sinal de fala que, posteriormente, sofre modificação e atraso devido à reflexão nas superfícies do ambiente e, finalmente, é captado e retorna ao seu emissor causando perturbação na conversação.

Os dispositivos empregados no cancelamento de eco acústico (AEC, do inglês Acoustic Echo Cancellation) são essenciais na garantia da qualidade da comunicação em sistemas viva-voz, tendo em vista a característica de depreciação da clareza na conversa introduzida pela presença do eco.

No presente trabalho propõe-se um sistema de cancelamento de eco acústico pré-filtrado (PAEC, do inglês Pre-Filtered AEC), que emprega um bloco de préprocessamento não-linear (NLP, do inglês Nonlinear Preprocessing) em cascata com um filtro adaptativo linear, definindo uma configuração de AEC com préfiltragem não-linear (NLPAEC, do inglês Non-linearly pre-Filtered AEC). Inicialmente, o bloco de NLP foi constituído de um modelo de alto-falante não-linear e com memória (NLML) baseado em um filtro de Volterra convencional com núcleos calculados a partir de um modelo físico que se mostrou preciso no modelamento de um alto-falante real. Assim, o sistema proposto NLPAEC(NLML) dispensaria o uso de um filtro adaptativo não-linear, com importante redução de complexidade.

Realizou-se um estudo do desempenho de algoritmos adaptativos lineares considerando condições de linearidade e não-linearidade, com o intuito de comprovar o ganho do sistema proposto em relação ao cancelamento linear convencional, assim como a superioridade do modelo não-linear adotado em relação ao modelo polinomial adotado na literatura.

O desempenho dos algoritmos foi analisado segundo os indicadores mais utilizados na literatura para a avaliação da performance de canceladores de eco, tais como: tempo de convergência, que fornece a informação de quão rápida é a adaptação do algoritmo; e redução do nível de eco acústico (ERLE).

Além do sistema proposto já descrito, foram considerados para fins de comparação o uso de AEC linear convencional com quatro tipos de alto-falantes: AEC(IL), com um alto-falante ideal; AEC(NLIL) com um modelo não-linear polinomial sem memória, largamente empregado na literatura para verificar o efeito da não-linearidade do alto-falante na adaptação de algoritmos de AEC; AEC(NLML), com o modelo não-linear do alto-falante mencionado acima e, finalmente, $\mathrm{AEC}(\mathrm{LL})$, com o alto-falante linear dado pelo núcleo de primeira ordem do mesmo modelo.

Também para fins de comparação, foi considerado o uso de AEC com préfiltragem linear com o modelo não-linear do alto-falante, LPAEC(NLML). 
Com a realização de simulações, pode-se observar um ganho considerável de ERLE alcançado pelo sistema proposto (NLPAEC) no caso do modelo de altofalante NLML. Portanto, a necessidade do uso do sistema proposto em casos de altas não-linearidades ficou evidenciada nessa primeira parte do presente trabalho.

Ainda que o projeto do bloco não-linear no sistema proposto pressuponha, idealmente, o conhecimento das características do alto-falante, a questão da escolha da implementação exata deste bloco permanecia em aberto, pois existe um compromisso importante entre complexidade computacional e desempenho do sistema, que precisava ser investigado neste contexto particular. A obtenção da resposta à essa questão foi o objetivo da segunda parte do presente trabalho.

A opção de implementação do bloco não-linear que resultaria na maior precisão na aproximação do alto-falante seria a utilização de recursos de integração numérica de equações diferenciais, mas a carga computacional desta implementação seria proibitiva para um sistema de cancelamento de eco prático. Assim, esse método de implementação do modelo não-linear foi empregado especificamente para a implementação do modelo físico do alto-falante a fim de se obter um cenário de simulação mais realista.

Adicionalmente ao filtro de Volterra Convencional empregado inicialmente no sistema proposto na primeira parte deste trabalho, outras possibilidades de implementação são estruturas alternativas relacionadas com o filtro de Volterra, tais como os modelos de Volterra Esparso Interpolado e PARAFAC-Volterra, além de discretizações pelo método de Euler das equações de espaço de estados do modelo físico real e de um sistema bilinear descrito em espaço de estados.

Com o objetivo de analisar o efeito do processo de adaptação no comportamento do sistema proposto foram realizadas simulações na condição de ausência de ruído, caso no qual o desempenho do cancelador é resultado apenas da ação do filtro adaptativo. Os resultados dessas simulações revelaram o fato de que o filtro adaptativo, mesmo que puramente linear, compensa pequenas imperfeições do modelo do alto-falante não-linear, tendo um papel fundamental no desempenho alcançado pelo cancelador de eco acústico não-linear proposto.

Baseando-se nas análises das simulações do sistema de cancelamento de eco acústico não-linear, que se utiliza dessas estruturas não-lineares alternativas como bloco de pré-processamneto não-linear, determinou-se que o modelo PARAFACVolterra de $2^{\mathrm{a}}$ ordem é a melhor opção para implementação do bloco NLP, pois apresenta melhor compromisso entre desempenho do sistema proposto e carga computacional.

Além disso, destaca-se o fato de que o filtro adaptativo, mesmo que puramente linear, compensa pequenas imperfeições do modelo do alto-falante não-linear, tendo um papel fundamental no desempenho alcançado pelo cancelador de eco acústico não-linear proposto. 


\section{ABSTRACT}

In hands-free communication systems, acoustic echo originates from the speech signal that subsequently undergoes modification and delay due to reflection on the surfaces of the environment, and finally is picked up and returned to its sender causing disturbance in the conversation.

Acoustic echo cancellers are essential in quality of communication in handsfree systems, due to the characteristic of depreciation of the clarity in the conversation introduced by the presence of the echo.

In the present work we propose a pre-filtered acoustic echo cancellation system (PAEC), which employs a cascade nonlinear preprocessor (NLP) block with a linear adaptive filter, defining an AEC configuration with non-linear pre-filtering (NLPAEC). Initially, the NLP block consisted of a non-linear loudspeaker model with memory (NLML) based on a conventional Volterra filter with kernels calculated from of a physical model that was used in the modeling of a real speaker. Thus, the proposed NLPAEC(NLML) system would dispense the use of a nonlinear adaptive filter, with important reduction of complexity.

We performed a study of the performance of linear adaptive algorithms considering linearity and non-linearity conditions, in order to prove the gain of the proposed system with respect to the conventional linear cancellation, as well as the superiority of the nonlinear model adopted in relation to the polynomial model adopted in the literature.

The performance of the algorithms was analyzed according to the most used indicators in the literature to evaluate the performance of echo cancellers, such as: convergence time, which provides information on how fast the algorithm is adapted; and reduction of the acoustic echo level (ERLE).

In addition to the proposed system already described, it was considered the use of conventional linear AEC with four types of loudspeakers: AEC(IL), with an ideal loudspeaker; AEC(NLIL) with a nonlinear model polynomial model without memory, widely used in the literature to verify the effect of the non-linearity of the loudspeaker in the adaptation of AEC algorithms; AEC(NLML), with the nonlinear loudspeaker model mentioned above and finally AEC(LL), with the linear loudspeaker given by the first-order kernel of the same model..

Also for purposes of comparison, it was considered the use of AEC with linear pre-filtering with the loudspeaker nonlinear model, LPAEC(NLML). With the realization of simulations, one can observe a considerable gain of ERLE reached by the proposed system (NLPAEC) in the case of the NLML loudspeaker model. Therefore, the need to use the proposed system in cases of high non-linearities was evidenced in this first part of the present work.

Although the design of the NLP block in the proposed system ideally pre- 
supposes the knowledge of the characteristics of the loudspeaker, the question of choosing the exact implementation of this block remained open, since there is an important compromise between computational complexity and performance of the system, which needed to be investigated in this particular context. Obtaining the answer to this question was the objective of the second part of the present work.

The option of NLP block implementation that would result in greatest precision in the loudspeaker model approximation would be the use of numerical integration features of differential equations, but the computational load of this implementation would be prohibitive for a practical echo cancellation system. Thus, this method of implementation of the nonlinear model was used specifically for the implementation of the physical model of the loudspeaker in order to obtain a more realistic simulation scenario.

In addition to the conventional Volterra filter already used in the system proposed in the first part of this work, other possibilities of implementation are alternative structures related to the Volterra filter, such as the Sparse-Interpolated Volterra Filter and PARAFAC-Volterra models, besides discretizations by the Euler method of the state-space equations of the real physical model and a bilinear system described in space of states.

In order to analyze the effect of the adaptation process on the behavior of the proposed system, simulations were performed in the no noise condition, in which case the performance of the canceller is only the result of the action of the adaptive filter. The results of these simulations have revealed the fact that the adaptive filter, even if purely linear, compensates the small imperfections of the loudspeaker nonlinear model, playing a key role in the performance achieved by the proposed non-linear acoustic echo canceller.

Based on the simulations of the non-linear acoustic echo cancellation system, using these alternative nonlinear structures as NLP block, it was determined that the 2nd order PARAFAC-Volterra model is the best option for implementation of the NLP block, since it presents the best compromise between performance of the proposed system and computational load.

In addition, the adaptive filter, even been purely linear, compensates small imperfections of the nonlinear loudspeaker model, playing a key role in the performance achieved by the proposed nonlinear acoustic echo canceller. 


\section{SUMÁRIO}

\section{Lista de Abreviaturas e Siglas}

1 Introdução 13

1.1 Aspectos Téoricos . . . . . . . . . . . . . . . 13

1.2 Objetivos . . . . . . . . . . . . . . . . . . 17

1.3 Organização . . . . . . . . . . . . . . . . . . . . . 20

2 Cancelamento de Eco 23

2.1 Eco Telefônico . . . . . . . . . . . . . . . . . . . . 24

2.2 Eco Acústico . . . . . . . . . . . . . . . . . . . . . . . . . 26

2.3 Cancelamento de Eco Acústico . . . . . . . . . . . . . . . . . . . 26

2.4 Filtragem Adaptativa . . . . . . . . . . . . . . . . . . . . . . 29

2.4.1 Filtro FIR Transversal . . . . . . . . . . . . . . . . . . . . 29

2.4 .2 Filtro de Wiener . . . . . . . . . . . . . 30

2.4.3 Filtro Adaptativo Transversal . . . . . . . . . . . . . 32

2.4.4 Algoritmos Adaptativos Lineares . . . . . . . . . . . . . 32

2.4.4.1 Least Mean Square - LMS . . . . . . . . . . . . 33

2.4.4.2 Normalized Least Mean Square - NLMS . . . . . 36

2.4.4.3 Affine Projection Algorithm - APA . . . . . . 39

2.4.4.4 Fast Block Least Mean Square - FBLMS . . . . . 41 
3.1 Modelo de Parâmetros Passivos para Alto-falantes Eletrodinâmicos 43

3.1.1 Alto-falantes Dinâmicos . . . . . . . . . . . . . . 44

3.1.2 Equações de espaço de estados . . . . . . . . . . . . . . . 44

3.1.3 Precisão do Modelo . . . . . . . . . . . . . . . . . . . 46

3.2 Modelo de Euler . . . . . . . . . . . . . . . . . . . . . . . . . . 48

3.2.1 Discretização pelo Método de Euler . . . . . . . . . . . 48

3.2.2 Carga Computacional . . . . . . . . . . . . . . . 49

3.3 Sistema Bilinear . . . . . . . . . . . . . . . . . . . . 51

3.3.1 Discretização do Sistema Bilinear . . . . . . . . . . . . 52

3.3.2 Carga Computacional . . . . . . . . . . . . . . 53

3.4 Filtro de Volterra Convencional . . . . . . . . . . . . . . . 54

3.4.1 Carga Computacional . . . . . . . . . . . . . 56

3.5 Filtro de Volterra Esparso Interpolado . . . . . . . . . . . . 58

3.5.1 Carga Computacional . . . . . . . . . . . . . . 62

3.6 Modelo PARAFAC-Volterra . . . . . . . . . . . . . . . . . . . 64

3.6.1 Carga Computacional . . . . . . . . . . . . . 66

4 Efeito de Não-Linearidades em Algoritmos de Cancelamento de $\begin{array}{ll}\text { Eco Acústico Linear } & 67\end{array}$

4.1 Condicionamento de Sinal e Velocidade de Convergência . . . . . 67

4.2 Indicadores de Desempenho . . . . . . . . . . . . . . . . . . 68 
4.2.1 Tempo de Convergência . . . . . . . . . . . . . . . 68

4.2 .2 Grau de Cancelamento . . . . . . . . . . . . . . . 68

4.3 Cancelador de Eco Acústico Linear . . . . . . . . . . . . . . . 69

4.4 AEC pré-filtrado - PAEC . . . . . . . . . . . . . . . 72

4.5 Análise da aplicação do modelo não-linear com memória . . . . 73

4.6 Simulação . . . . . . . . . . . . . . . . . . . . . 76

4.7 Resultados Obtidos . . . . . . . . . . . . . . . . . 80

4.8 Comparação de desempenho dos Algoritmos . . . . . . . . . . . . 87

4.9 Conclusões . . . . . . . . . . . . . . . . . . . . . . . . . . . . . . . . 88

$\begin{array}{llr}5 & \text { Sistema Proposto } & 90\end{array}$

5.1 Efeito da Adaptação . . . . . . . . . . . . . . . . . . . . . . . 91

5.2 Pré-Processamento Não-Linear . . . . . . . . . . . . . . . . . . . . . . 92

5.2 .1 Sistema Proposto Ideal . . . . . . . . . . . . . . . . . . 93

5.2.2 Resultados Obtidos . . . . . . . . . . . . . . . . . . 93

5.2.3 Comparação de desempenho dos Algoritmos . . . . . . . . 98

5.2.4 Conclusões . . . . . . . . . . . . . . . . . . . . . . . . . . 98

5.3 Compromisso entre Desempenho e Complexidade Computacional 99

5.3.1 Desempenho do Sistema Proposto . . . . . . . . . . . 100

5.3.2 Carga Computacional dos Modelos . . . . . . . . . . 125

6 Conclusões 
Apêndice A - Canceladores de Eco Acústico

Apêndice B - Algoritmo LMS

Apêndice C - Algoritmo NLMS

Apêndice D - Algoritmo FBLMS

Apêndice E - Algoritmo APA

Apêndice F - Modelo de Parâmetros Passivos

F.1 Modelo de Parâmetros Passivos . . . . . . . . . . . . . . . 152

F.2 Indutância Não-Linear . . . . . . . . . . . . . . . . . . . . . . 153

F.3 Transdutor de 2-Portas . . . . . . . . . . . . . . . . . . 154

F.4 Circuito Elétrico . . . . . . . . . . . . . . . . . 156

F.5 Equações de espaço de estados . . . . . . . . . . . . . . . . 158

Apêndice G - Algoritmo Bilinear Esparso 


\section{LISTA DE ILUSTRAÇÕES}

1 Diagrama do sistema de comunicação. . . . . . . . . . . . . . . . 28

2 Circuito Híbrido da RPTC. Extraído de [22] . . . . . . . . . . . . 30

3 Sistema de cancelamento de eco acústico com um filtro adaptativo. 32

4 Diagrama de um Sistema Adaptativo para identificação de sistemas. 34

$5 \quad$ Estrutura FIR Transversal. . . . . . . . . . . . . . . . . . . 35

6 Filtro Adaptativo Transversal. . . . . . . . . . . . . . . . . 38

$7 \quad$ Filtro adaptativo em Bloco. . . . . . . . . . . . . . . 46

8 Esquema seccional de um alto-falante eletrodinâmico. Adaptado de $[34] \ldots \ldots \ldots \ldots$

9 Valores numéricos dos parâmetros do modelo do alto-falante. Extraído de [19]. . . . . . . . . . . . . . . . . . . . . . . 51

10 Valores estimados para a aproximação de $L\left(x_{1}\right)$. Extraído de [18]. 52

11 Sinais de saída do alto-falante real e modelo passivo. Extraído de

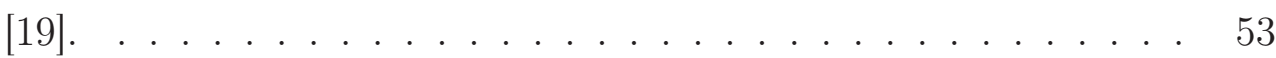

12 Esquema esparso com interpolação para aproximação de um filtro

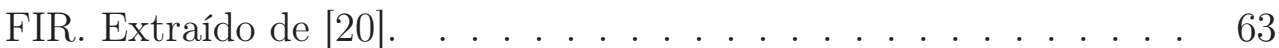

13 Diagrama em blocos do Filtro de Volterra Esparso Interpolado.

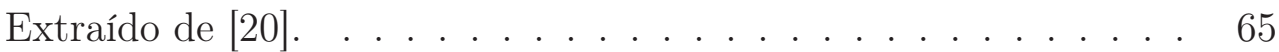

14 Modelo PARAFAC-Volterra. Extraído de [18]. . . . . . . . . . . . 70

15 LAEC com alto-falante ideal. . . . . . . . . . . . . . . . 75 
16 LAEC usando um modelo do alto-falante. . . . . . . . . . . 76

17 LPAEC(LL). . . . . . . . . . . . . . . . . . 77

$18 \quad$ LPAEC(NLML). . . . . . . . . . . . . . . . . . . . . 78

19 Índices de ajuste de ordem p de um Filtro de Volterra Convencional. Extraído de [18]. . . . . . . . . . . . . . . . . . . . . 80

20 Fluxograma genérico dos sistemas LAEC simulados. . . . . . . . . 83

21 ERLE do AEC(IL) e AEC(NLIL) . . . . . . . . . . . 86

22 ERLE do AEC(NLIL), $\beta=0 \ldots \ldots . \ldots . \ldots 87$

$23 E R L E$ do AEC(NLIL), $\alpha=0 \ldots \ldots \ldots 88$

$24 \quad E R L E$ do $\mathrm{AEC}(\mathrm{IL})$ e $\mathrm{AEC}(\mathrm{LL}) \ldots \ldots \ldots . \ldots . \ldots . . \ldots 89$

$25 E R L E$ do $\mathrm{AEC}(\mathrm{IL})$ e $\mathrm{AEC}(\mathrm{NLIL}) \ldots \ldots . . \ldots 90$

26 ERLE do AEC(LL) e AEC(NLML). . . . . . . . . . . . . . . 91

27 Cancelador de eco acústico com NLP. . . . . . . . . . . . . . . . . 95

28 NLPAEC(NLML). . . . . . . . . . . . . . . . . . . . . . . . . . . 998

29 ERLE do NLPAEC(NLML) e AEC(NLML). . . . . . . . . 100

30 ERLE do NLPAEC(NLML) e LPAEC(NLML) . . . . . . . . . . 101

31 ERLE do NLPAEC(NLML) e AEC(IL). . . . . . . . . . . . . 102

32 Cancelador de eco não-linear proposto - NLPAEC(NLML). . . . . 105

33 Setup 1: ERLE do NLPAEC(NLML) com diferentes blocos NLP Janela=1s. . . . . . . . . . . . . . . . . . . . 109

34 Setup 1: ERLE do NLPAEC(NLML) com diferentes blocos NLP Janela=0.1s. . . . . . . . . . . . . . . . . . . . . 110 
35 Setup 1: ERLE do NLPAEC(NLML) sem Ruído.

36 Setup 1: Precisão de diferentes blocos NLP ao representar o AltoFalante. . . . . . . . . . . . . . . . . 112

37 Setup 2: ERLE do NLPAEC(NLML) com diferentes blocos NLP Janela $=1 \mathrm{~s}$

38 Setup 2: ERLE do NLPAEC(NLML) com diferentes blocos NLP Janela=0.1s. . . . . . . . . . . . . . . . . . . . . 116

39 Setup 2: ERLE do NLPAEC(NLML) sem Ruído. . . . . . . . . . 117

40 Setup 2: Precisão de diferentes blocos NLP ao representar o AltoFalante.

41 Setup 3: ERLE do NLPAEC(NLML) com diferentes blocos NLP Janela $=1 \mathrm{~s}$.

42 Setup 3: ERLE do NLPAEC(NLML) com diferentes blocos NLP Janela $=0.1$ s. . . . . . . . . . . . . . . . . . . . . . . . . 121

43 Setup 3: ERLE do NLPAEC(NLML) sem Ruído. . . . . . . . . . 122

44 Setup 3: Precisão de diferentes blocos NLP ao representar o AltoFalante.

45 Setup 4: ERLE do NLPAEC(NLML) com diferentes blocos NLP Janela $=1 \mathrm{~s}$

46 Setup 4: ERLE do NLPAEC(NLML) com diferentes blocos NLP Janela=0.1s. . . . . . . . . . . . . . . . . . . . 126

47 Setup 4: ERLE do NLPAEC(NLML) sem Ruído. . . . . . . . . . 127

48 Setup 4: Precisão de diferentes blocos NLP ao representar o AltoFalante. 
49 AEC(IL) . . . . . . . . . . . . . . . . . . . . . . . 142

$50 \quad$ LPAEC(LL). . . . . . . . . . . . . . . . . . . . . . . . . . 142

$51 \quad$ AEC(NLIL) . . . . . . . . . . . . . . . . . . . 143

$52 \quad$ AEC(NLML) . . . . . . . . . . . . . . . . . . . . 143

$53 \quad$ LPAEC(NLML). . . . . . . . . . . . . . . . . . . . 143

$54 \quad$ NLPAEC(NLML). . . . . . . . . . . . . . . . . . . . . . 144

55 Circuito representando uma indutância não-linear controlada por corrente. Extraído de [19]. . . . . . . . . . . . . . . . . . . . . . . 159

56 Circuito referente a bobina em movimento no campo magnético. Extraído de [19]. . . . . . . . . . . . . . . . . . . . . . . . . 160

57 Modelo de circuito elétrico de um alto-falante eletrodinâmico. Ex-

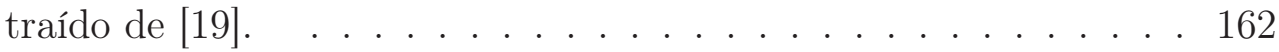




\section{LISTA DE TABELAS}

1 Etapas de implementação do algoritmo LMS [29].

2 Etapas de implementação do algoritmo NLMS [29].

3 Etapas de implementação do algoritmo APA [29].

4 Etapas de implementação do algoritmo FBLMS [29].

$5 \quad$ Número de operações do modelo de Euler.

6 Número de operações do Sistema Bilinear

7 Equações de Complexidade Estrutural e Carga Computacional. . .

8 Análise da carga computacional dos modelos. . . . . . . . . . . .

9 Carga computacional e valores de ERLE dos modelos. . . . . . . . 


\section{LISTA DE ABREVIATURAS E SIGLAS}

\begin{tabular}{|c|c|}
\hline $\mathrm{AEC}$ & Acoustic Echo Cancellation \\
\hline APA & Affine Projection Algorithm \\
\hline NAE & Normalized Approximation Error \\
\hline ERLE & Echo Return Loss Enhancement \\
\hline FBLMS & Fast Block Least Mean Square \\
\hline FIR & Finite Impulse Response \\
\hline IL & Ideal Loudspeaker \\
\hline LAEC & Linear Acoustic Echo Cancellation \\
\hline LL & Linear Loudspeaker \\
\hline LMS & Least Mean Square \\
\hline LPAEC & Linearly pre-Filtered AEC \\
\hline NLAEC & Nonlinear Acoustic Echo Cancellation \\
\hline NLIL & Nonlinear Instantaneous Loudspeaker \\
\hline NLML & Nonlinear and with Memory Loudspeaker \\
\hline NLMS & Normalized Least Mean Square \\
\hline NLP & Nonlinear Preprocessing \\
\hline NLPAEC & Non-linearly pre-Filtered AEC \\
\hline PAEC & Pre-Filtered AEC \\
\hline PARAFAC & Parallel Factor \\
\hline
\end{tabular}


PVM PARAFAC Volterra Model

PSTN Public Switched Telephone Network

SPI-VF Sparse Interpolated Volterra Filter

TDLMS Transform Domain Least Mean Square

VF Volterra Filter 


\section{INTRODUÇÃO}

O cancelamento de eco acústico é um aspecto importante do projeto de sistemas de comunicação, tais como: sistemas de telefonia fixa, sistemas viva-voz, sistemas móveis (sem fio) ou sistemas de teleconferência, pois o eco acústico pode degradar a qualidade do serviço nestes sistemas.

Sendo assim, na próxima seção são apresentados os aspectos teóricos básicos sobre o eco acústico, a contextualização do problema do cancelamento de eco acústico em sistemas de telecomunicação e uma análise dos estudos científicos atuais que abordam tal temática. Nas seções seguintes, os objetivos do presente trabalho são definidos e uma breve descrição de cada capítulo é apresentada a fim de revelar como o trabalho é organizado.

\section{$1.1 \quad$ Aspectos Téoricos}

O eco acústico ocorre quando o som emitido por uma fonte sonora é refletido e modificado (atenuado, distorcido, etc...) pelo meio em que se propaga, resultando após um determinado intervalo de tempo na recepção do som original adicionado a uma versão refletida e distorcida desse som, originando uma característica de repetição do som emitido. Os efeitos do eco acústico dependem do tempo de atraso entre a incidência e reflexão das ondas, a amplitude das ondas refletidas e o número de caminhos através dos quais as ondas são refletidas.

Em um equipamento de comunicação em modo viva-voz, o acoplamento acús- 
tico entre o alto-falante e o microfone em uma das pontas da conexão provoca um eco indesejado na outra ponta da conexão. O cancelamento adaptativo de tal eco é um problema tradicionalmente abordado em processamento digital de sinais e, geralmente, o conjunto formado pelo amplificador, alto-falante, ambiente reverberante e microfone é modelado por um sistema linear [1].

Limitações no desempenho dos canceladores de eco adaptativos (AEC, do inglês Acoustic Echo Cancellation) para terminais viva-voz são produzidas por ruído, efeitos de truncamento e precisão finita, truncamento da resposta acústica impulsiva, vibração do ambiente reverberante, não-linearidades do auto-falante, convergência, rastreamento, e double-talk [2].

Especialmente, a miniaturização dos transdutores introduz distorções nãolineares que afetam a performance dos canceladores de eco lineares (LAEC, do inglês Linear Acoustic Echo Cancellation) [3]. Assim, se torna interessante estudar sua robustez na presença de não-linearidades [4].

Mesmo sendo raro na literatura científica, alguns trabalhos foram realizados com o intuito de analisar os canceladores de eco lineares considerando as nãolinearidades provenientes do alto-falante, dentre os quais pode-se citar $[2,5,6]$.

Em [2] foi feito um resumo dos fatores limitantes dos canceladores de eco lineares em um sistema de comunicação viva-voz, além de ter sido apresentado um estudo sobre o efeito da não-linearidade do alto-falante e da vibração do ambiente reverberante na melhoria da perda de retorno de eco (ERLE, do inglês Echo Return Loss Enhancement) em regime permanente.

A atenuação do eco obtida pelos canceladores de eco que usam algoritmos adaptativos lineares na presença de distorções causadas pelo alto-falante foi discutida em [6]. Em particular, nesse trabalho compara-se os algoritmos NLMS, APA e QR-RLS considerando o caminho do eco como "quase" linear ou não- 
linear, e fazendo uso de um modelo polinomial sem memória para representar as distorções causadas pelo alto-falante.

A fim de analisar os canceladores de eco lineares na presença de nãolinearidades, [4] apresenta a avaliação de robustez de alguns algoritmos adaptativos lineares considerando o mesmo modelo não-linear do alto-falante usado em $[6,7]$.

O caminho do eco acústico consiste basicamente da cascata de um amplificador, alto-falante e microfone, além de conter o caminho de propagação acústica entre esses dois últimos componentes [8]. Em geral, dois tipos de distorções nãolineares são consideradas na literatura para fins de estudo de cancelamento de eco acústico não-linear: a não-linearidade que é proveniente do amplificador em situação de saturação [9] e a não-linearidade inerente ao alto-falante [3, 10].

Estruturas em cascata, filtros de potências e filtros de Volterra de $2^{\text {a }}$ ordem aplicados ao cancelamento de eco acústico adaptativo são discutidos em [8]. Em particular, esse estudo demonstra que a maioria das abordagens adotadas usam modelos simplificados do caminho de eco acústico, caracterizando tais esquemas como não realistas uma vez que não consideram cenários de altas não-linearidades do acoplamento alto-falante-microfone.

Uma comparação entre estruturas não-lineares em cascata e modelos de Volterra é realizada em [11]. Nesse artigo as características dos modelos de Hammerstein, Wiener e Volterra são discutidas e o número de paramêtros relacionado à implementação de cada estrutura é apresentado. A complexidade computacional associada ao modelo de Volterra é revelada pelos dados apresentados e, por isso, simulações de cancelamento de eco são realizadas somente com os modelos em cascata. Por fim, é constatado o melhor desempenho da estrutura não-linear de Hammerstein com base nos resultados dessas simulações. 
Estruturas de Wiener e Hammerstein são comparadas em maiores detalhes em [12], sendo que são utilizadas funções não-lineares sigmoid e spline parametrizadas, cujas formas podiam ser alteradas durante o processo de aprendizado. Novamente, o melhor desempenho do modelo de Hammerstein foi constatado.

Entretanto, é difícil garantir a convergência para a solução ideal ou mesmo assegurar comportamento de adaptação para estruturas adaptadas em cascata [8]. Contudo, caso as não-linearidades envolvidas sejam advindas de uma característica de saturação sem memória pode-se considerar o filtro de potências, o qual consiste de uma estrutura adaptável de potências em paralelo que modelam o caminho de eco não-linear $[9,5,13]$.

Filtros de Volterra podem ser usados para representar uma ampla variedade de sistemas não-lineares com memória [14], tornando-o útil no contexto de cancelamento de eco acústico em situações em que as não-linearidades consideradas são provenientes do alto-falante.

Os artigos $[3,15]$ usam um Filtro de Volterra de $2^{\mathrm{a}}$ ordem para modelar as não linearidades do alto-falante em dois esquemas distintos: o primeiro se utiliza de uma configuração de Hammerstein onde o Filtro de Volterra Adaptativo de $2^{\mathrm{a}}$ ordem antecede um filtro adaptativo linear; e o segundo modela o caminho de eco acústico integralmente através de um Filtro de Volterra de $2^{\mathrm{a}}$ ordem.

As não-linearidades do alto-falante são investigadas em [16] através da proposta de uma arquitetura não-linear baseada em funções adaptativas spline com custo computacional reduzido da ordem de $\mathrm{O}(\mathrm{N})$. Apesar do esquema proposto baseado em splines apresentar boa performance, os experimentos realizados utilizaram ruído gaussiano branco como entrada de excitação ao invés de sinais reais de fala tornando o resultado não realista.

Como discutido em [17] e, em maior detalhe em [18], uma deficiência recor- 
rente nas abordagens ao problema de cancelamento não-linear de eco acústico (NLAEC, do inglês Nonlinear Acoustic Echo Cancellation) existentes na literatura é a adoção sem justificativa satisfatória de modelos não-lineares particulares, de estruturas de filtragem decorrentes destes modelos e de parâmetros de tais estruturas. Como forma de sanar esta deficiência, os dois trabalhos mencionados propõem uma metodologia que faz uso de um modelo físico da não-linearidade em questão para calcular, de forma eficiente, seus núcleos de Volterra. A partir destes núcleos de referência, as diferentes alternativas de modelamento e de filtragem podem ser cotejadas com maior embasamento.

Em particular, em $[18,17]$ foi apresentado um estudo de caso utilizando um modelo físico não-linear que se mostrou preciso no modelamento de um altofalante real [19].

\subsection{Objetivos}

O cancelamento de eco acústico não-linear (NLAEC) será analisado através de um sistema proposto que emprega um bloco de pré-processamento não-linear (NLP) em cascata com um filtro adaptativo linear. Será realizado inicialmente um estudo do desempenho de algoritmos adaptativos lineares considerando condições de linearidade e não-linearidade, com o intuito de comprovar o ganho do sistema proposto em relação ao cancelamento linear convencional, assim como a superioridade do modelo não-linear adotado em relação ao modelo polinomial adotado na literatura.

Nessa primeira parte do presente trabalho, alguns canceladores de eco acústico lineares serão simulados considerando fatores relevantes para a caracterização dos mesmos, tais como: respostas impulsivas acústicas reais, caminho de eco linear e na presença de não-lineridade do alto-falante. A representação dessa 
não-linearidade se dará de forma distinta através de dois modelos: o primeiro será o modelo polinomial sem memória já utilizado por $[4,6,7]$; e o segundo será o modelo apresentado em $[17,18]$, que se utiliza de núcleos de Volterra calculados de forma eficiente e que representam um modelo físico preciso de um alto-falante real.

Os sistemas de cancelamento de eco acústico linear (LAEC) a serem simulados são descritos a seguir: O primeiro sistema considerará o caminho do eco acústico como sendo linear, representando dessa forma o cancelamento linear convencional. O segundo sistema usará o modelo não-linear polinomial sem memória com o objetivo de analisar o efeito da não-linearidade do alto-falante na adaptação do algoritmo. Finalmente, o terceiro sistema empregará o modelo de parâmetros passivos para alto-falantes dinâmicos proposto em [19] e que se mostrou preciso no modelamento de um modelo físico real. Esse modelo será implementado através do cálculo de suas equações de espaço de estado utilizando o método de resolução de equações diferenciais ordinárias realizada pela função ode15s do Matlab.

Adicionalmente, o cancelamento de eco acústico não-linear (NLAEC) será analisado através da simulação do sistema proposto, já mencionado anteriormente, que emprega um bloco de pré-processamento não-linear (NLP) em cascata com um filtro adaptativo linear. Essa configuração de cancelador de eco acústico prevê a representação do alto-falante através de um modelo não-linear que será implementado como bloco de NLP, o qual será constituído pelo modelo de parâmetros passivos do alto-falante implementado pela função $O D E 15 s$, ou seja, pelo próprio modelo do alto-falante. Essa configuração do sistema proposto permite a análise de desempenho no caso ideal, no qual o filtro adaptativo converge para a resposta impulsiva da sala.

Esses três canceladores de eco acústicos lineares e o sistema proposto, nãolinear, serão analisados através do uso de quatro algoritmos adaptativos lineares 
(LMS, NLMS, FBLMS e APA) e os desempenhos dos sistemas simulados serão verificados considerando os indicadores mais utilizados na literatura: ERLE e Tempo de Convergência. Sendo que essa análise será realizada com o intuito de comprovar o ganho do sistema proposto em relação ao cancelamento linear convencional, assim como a superioridade do modelo não-linear adotado em relação ao modelo polinomial adotado na literatura.

O projeto do bloco não-linear no sistema proposto pressupõe, idealmente, o conhecimento das características do alto-falante, porém a questão da escolha da implementação exata deste bloco permanece em aberto, pois existe um compromisso importante entre complexidade computacional e desempenho do sistema, que precisa ser investigado neste contexto particular.

Consequentemente, na segunda parte do presente trabalho, além do filtro de Volterra convencional já empregado inicialmente no sistema proposto, outras possibilidades de implementação do bloco NLP seriam alguma das estruturas alternativas relacionadas com o filtro de Volterra e as discretizações pelo método de Euler das equações de espaço de estados do modelo físico real [19] e de um sistema bilinear descrito em espaço de estados [17].

As estruturas não-lineares alternativas ao modelo de Volterra Convencional, e derivadas do mesmo, são os modelos de Volterra Esparso Interpolado e PARAFAC-Volterra. O primeiro consiste de uma simplificação do modelo de Volterra Convencional através da redução das suas extensões de memórias de acordo com um fator de interpolação, o qual a priori é utilizado para o descarte de alguns coeficientes dos núcleos do modelo, porém que é utilizado a posteriori para recuperação das amostras da saída do modelo relativas aos coeficientes desprezados [20]. O último é derivado da decomposição de fator paralelo (PARAFAC, do inglês Parallel Factor) dos núcleos de Volterra de ordem maior que 2, os quais são considerados como tensores simétricos [21]. Teoricamente, essas duas 
estruturas não-lineares resultam em uma redução de complexidade substancial com relação ao modelo de Volterra Convencional, por isso estão sendo cotejadas no presente trabalho.

Por fim, a análise do desempenho do sistema de cancelamento de eco acústico não-linear (NLAEC) e da carga computacional de cada uma das estruturas nãolineares alternativas que implementa o bloco NLP, servirão de base para o alcance do objetivo do presente trabalho, que é a determinação de qual dessas opções de implementação resultará em um melhor compromisso entre carga computacional e precisão na aproximação do alto-falante.

\subsection{Organização}

Esse capítulo descreve de uma forma geral o problema do cancelamento de eco acústico em sistemas de telecomunicações, expõe os objetivos e apresenta como é estruturado o presente trabalho.

O Capítulo 2 cita a origem do cancelamento de eco, explica os diferentes tipos de eco encontrados em sistemas de comunicação e apresenta os motivos que justificam a utilização de dispositivos canceladores de eco. Além disso, esse capítulo identifica quais são os principais desafios do cancelamento de eco acústico e aborda conceitos de filtragem adaptativa, mencionando a estrutura transversal do filtro de respota finita ao impulso (FIR, do inglês Finite Impulse Response), a solução ótima de Wiener, o filtro adaptativo e os algoritmos adaptativos lineares mais utilizados na literatura para implementação de canceladores de eco.

O Capítulo 3 apresenta o modelo de parâmetros passivos para alto-falantes eletrodinâmicos na forma de equações de espaço de estados, além de mencionar algumas opções de estruturas não-lineares que se aproximam desse modelo, a fim de representar o alto-falante atuando como bloco de pré-processamento não- 
linear em canceladores de eco acústico não-lineares. A complexidade estrutural de cada estrutura não-linear é analisada e a expressão para cálculo de sua carga computacional é apresentada.

O Capítulo 4 revela o comportamento dos canceladores de eco lineares na presença de não-linearidades através de simulações que utilizam dois modelos não-lineares do alto-falante. Essas simulações são implementadas por scripts do Matlab, os resultados obtidos são apresentados na forma de parâmetros usualmente empregados como indicadores de performance na análise dos dispositivos canceladores de eco acústico e, adicionalmente, o conceito de condicionamento de sinal é introduzido.

Por fim, expõe-se as considerações sobre a performance dos diferentes canceladores de eco lineares simulados e comentários sobre a comparação do desempenho dos algoritmos adaptativos empregados em tais sistemas.

O Capítulo 5 descreve o sistema cancelador de eco acústico proposto que consiste de uma estrutura em cascata que se utiliza de uma etapa de préprocessamento não-linear seguida por um filtro FIR adaptativo.

Inicialmente, o efeito do processo de adaptação é analisado e o sistema proposto é simulado em um cenário ideal, onde o bloco de pré-processamento nãolinear é o próprio modelo do alto-falante.

Em seguida, as estruturas não-lineares alternativas são consideradas como bloco NLP dando origem a diferentes configurações do sistema proposto e os diferentes setups e cenários considerados nas simulações são descritos anteriormente à apresentação dos resultados obtidos.

As análises de desempenho do cancelamento de eco acústico dos sistemas simulados e a carga computacional de cada estrutura não-linear utilizada são apresentados, sendo que essas informações servem de base para a determinação 
do compromisso entre desempenho e carga computacional de cada modelo nãolinear cotejado.

O Capítulo 6 trata do resultado das análises sobre o comportamento do sistema proposto empregando diferentes estruturas não-lineares. Em especial, a análise criteriosa do compromisso entre desempenho e carga computacional associado a cada estrutura não-linear serve de base para a decisão de qual estrutura não-linear apresenta melhor comprommisso e deve ser considerada como bloco NLP do sistema proposto. 


\section{CANCELAMENTO DE ECO}

O cancelamento de eco foi desenvolvido no início da década de 1960 pela AT\&T Bell Labs e mais tarde pela COMSAT TeleSystems [22]. Mais precisamente, a história do concelamento de eco se inicia em 10 de Julho de 1962 com o lançamento do primeiro satélite de telecomunicações pela empresa Bell Laboratories $[23]$.

Existem dois tipos de ecos em sistemas de telecomunicação: o eco telefônico e o eco acústico [22]. Um diagrama do sistema de comunicação utilizado durante uma chamada telefônica entre um telefone fixo e um celular é apresentado na Figura 1.

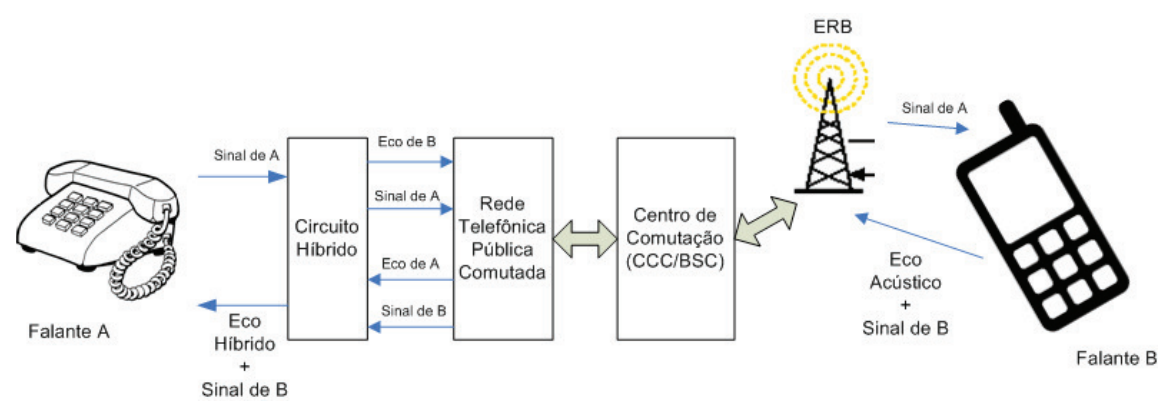

Figura 1: Diagrama do sistema de comunicação.

O eco telefônico resulta do descasamento de impedâncias devido à estrutura híbrida do sistema de telefonia, onde o sistema de 2 fios do assinante é conectado a um sistema de 4 fios da rede de telecomunicação. Em razão disso, esse tipo de eco também é chamado de eco híbrido ou de linha.

O eco acústico resulta de um caminho de retorno (feedback) estabelecido entre 
alto-falante e microfone em um telefone celular, sistema viva-voz ou sistema de teleconferência. O eco acústico pode ser refletido por diferentes superfícies e viaja através de diferentes caminhos.

Os ecos telefônico e acústico são indesejáveis e podem ser perturbadores em sistemas de comunicação. Assim, o uso de algum tipo de dispositivo que tenha por objetivo a eliminação de tais ecos durante a realização de uma chamada telefônica se faz necessário. Daí surge a aplicabilidade do dispositivo cancelador de eco, que basicamente é um filtro que modela adaptativamente a resposta ao impulso do caminho de eco, resultando na identificação do sistema real e gerando uma estimativa do sinal desejado que é empregada no cancelamento do eco.

\subsection{Eco Telefônico}

O eco telefônico é a principal fonte de eco gerado a partir da Rede Telefônica Pública Comutada (PSTN, do inglês Public Switched Telephone Network) [22]. Ecos de uma linha telefônica são devidos à reflexão dos sinais nos pontos de descasamento de impedâncias na conexão dos circuitos. Para estabelecer uma chamada de longa distância, em cada extremidade do sistema de comunicação, a linha de assinante de 2 fios deve ser ligada a uma linha de 4 fios, tal como ilustrado na Figura 2.

O dispositivo que conecta o circuito do assinante de 2 fios à linha de 4 fios é chamado de Híbrido, que é basicamente um circuito em ponte de três portas. Se a ponte híbrida for perfeitamente equilibrada, então não haveria nenhuma reflexão

ou eco. No entanto, cada circuito Híbrido serve um determinado número de linhas de assinantes. Nem todas as linhas dos assinantes apresentam as mesmas características de comprimento e de impedância e por isso não é possível alcançar o equilíbrio perfeito para todos os assinantes nos Híbridos. Quando a ponte não 


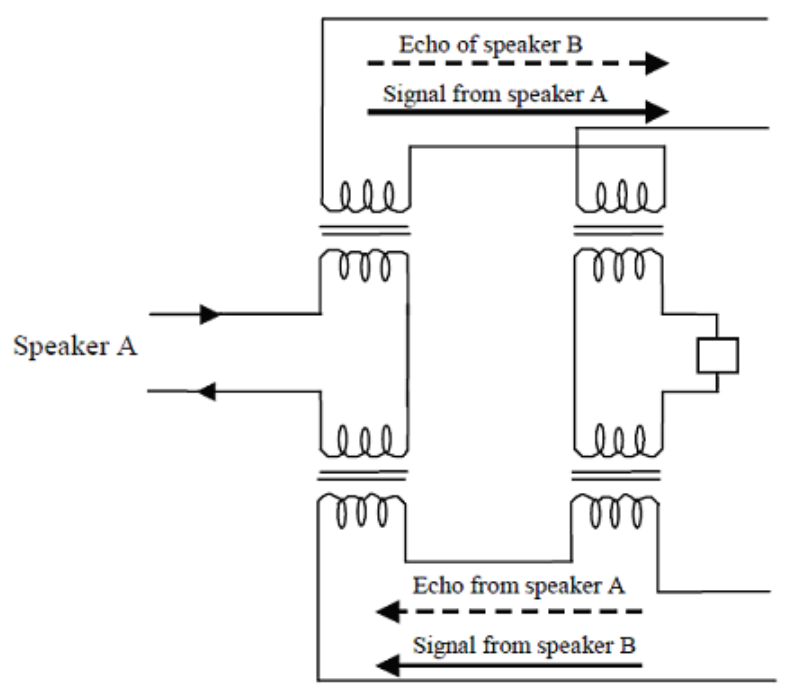

Figura 2: Circuito Híbrido da RPTC. Extraído de [22].

é perfeitamente equilibrada, uma parte da energia do sinal recebido pelas linhas de 4 fios é rebatida de volta sobre si mesma e produz um eco.

O eco é frequentemente medido em termos de perda de retorno de eco: quanto maior for a perda de retorno do eco menor será o eco. Os ecos das linhas telefônicas são indesejáveis, e tornam-se mais irritantes quando a amplitude do eco é relativamente alta e o atraso do eco é longo. Por exemplo, quando uma chamada à longa distância é feita através de um satélite, o atraso do eco de ida e volta pode ser tão longo quanto 600ms, e os ecos podem tornar-se perturbadores [24].

Em sistemas de telefonia móvel digital, os sinais de voz são processados nos dois pontos da rede: primeiro no codificador de voz do telefone celular os sinais de fala são digitalizados, comprimidos, codificados e modulados, e então, processados na interface de rádio frequência da rede. O atraso total introduzido pelas diferentes fases de processamento de sinal digital é de $80 \mathrm{~ms}$ até $100 \mathrm{~ms}$, o que resulta em um atraso total de ida e volta de 160 até $200 \mathrm{~ms}$ para qualquer eco. Um atraso dessa magnitude vai fazer qualquer eco considerado ser prejudicial para o processo de comunicação. Devido ao retardo de processamento inerente em sistemas de comunicações móveis digitais, é essencial e obrigatório empregar 
cancelamento de eco nas centrais de comutação de telefonia móvel [22].

\subsection{Eco Acústico}

O eco acústico se origina do acoplamento entre alto-falante e microfone do terminal telefônico, acoplamento esse que é tipicamente muito maior em um terminal operando no modo viva-voz. Devido à resposta do alto-falante não ser perfeitamente linear, o eco acústico tem uma parcela não-linear que pode ser relevante frente à parcela linear. Após ser emitido pelo alto-falante, o som é refletido nas superfícies do ambiente e a superposição destas diversas reflexões é captada pelo microfone. Esta resposta acústica do ambiente pode ser modelada como um sistema linear com uma determinada resposta ao impulso. Devido às contínuas mudanças no ambiente acústico (por exemplo, pessoas e/ou objetos em movimento), a resposta ao impulso é uma função do tempo e o sistema linear correspondente é variante no tempo [13].

Em comparação com o eco telefônico, o eco acústico tem uma potência maior e um caminho de eco mais longo, que também é variável no tempo. Portanto, a remoção de eco acústico é mais desafiadora do que a remoção de eco telefônico. $\mathrm{Na}$ verdade, o eco telefônico pode ser considerado como um caso especial de eco acústico onde o caminho de eco é curto e invariante no tempo.

\subsection{Cancelamento de Eco Acústico}

O cancelamento de eco acústico é geralmente realizado através de um filtro adaptativo que produz uma réplica do sinal de eco acústico. Essa réplica é então subtraída do sinal do microfone, antes da sua transmissão através da rede de comunicação, de modo que o eco seja removido. A representação de um cancelador de eco acústico usando um filtro adaptativo é mostrada na Figura 3. 


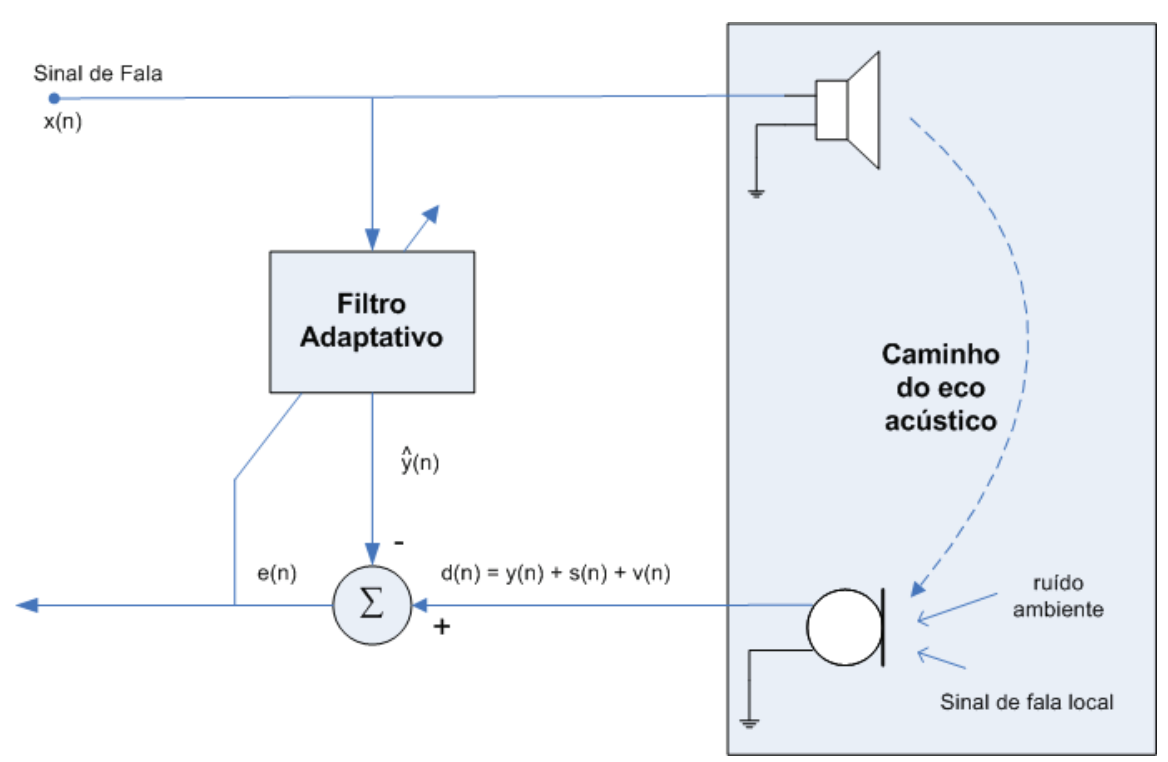

Figura 3: Sistema de cancelamento de eco acústico com um filtro adaptativo.

Cada dispositivo viva-voz em uma conexão telefônica precisa de seu próprio cancelador de modo a impedir que o eco acústico nele produzido seja transmitido para a outra extremidade. Portanto, quando os dispositivos de viva-voz são utilizados em ambas as extremidades da conexão, são necessários dois canceladores de eco acústico. Sem perda de generalidade, a Figura 3 mostra apenas um dispositivo AEC de uma das extremidades da conexão e os sinais mencionados no sistema são descritos como segue:

- $x(n)$ é o sinal produzido por um usuário na outra extremidade da conexão e recebido pelo sistema AEC.

- $d(n)$ é o sinal desejado do sistema AEC e consiste no seguinte: $-y(n)$ é o sinal do acoplamento alto-falante-microfone, o qual usualmente é chamado de eco acústico. $-s(n)$ é o sinal produzido pelo usuário nessa extremidade da conexão. $-v(n)$ é o sinal de ruído ambiente .

- $e(n)$ é o sinal de erro do sistema AEC. Esse sinal é transmitido para a outra extremidade da conexão e é composto pelo eco residual somado às 
componentes $\mathrm{s}(\mathrm{n})$ e $\mathrm{v}(\mathrm{n})$, as quais idealmente não deveriam ser modificadas pela ação do sistema AEC.

- $\hat{y}(n)$ é a estimativa do sinal de eco acústico.

Os principais desafios do cancelamento de eco acústico são os seguintes: o caminho de eco acústico pode ser muito longo, chegando a centenas de milisegundos para uma sala de escritório por exemplo; e a fala é um sinal altamente correlacionado e não-estacionário [25], dificultando a convergência do filtro adaptativo.

Para lidar com o primeiro desafio, um filtro adaptativo FIR com centenas ou milhares de coeficientes é utilizado para representar o caminho de eco. Isso resulta em um custo computacional elevado, mesmo para os algoritmos adaptativos mais simples como LMS e NLMS. Afim de reduzir essa complexidade computacional elevada algumas técnicas de processamento de sinais digitais são utilizadas, tais como: implementação de algoritmos em blocos e/ou no domínio da frequência [26].

Para lidar com o segundo desafio, são necessários filtros adaptativos com maior velocidade de convergência e/ou rastreamento. Dentre esses, o algoritmo de projeção afim (APA, do inglês Affine Projection Algorithm) pode ser usado, pois o mesmo possui taxa de convergência elevada quando excitado por sinais de fala [27].

Abordagens padrão de filtragem AEC linear obtiveram desempenho de cancelamento satisfatório ao considerar o caminho do eco puramente linear [1]. Entretando, se distorções não-lineares não desprezíveis são introduzidas no sinal $y(n)$, a efetividade dessas estruturas de filtro decai consideravelmente e então métodos de AEC não-linear são necessários [28]. 


\subsection{Filtragem Adaptativa}

O objetivo de um filtro adaptativo é minimizar uma função custo do erro e(n) entre sua saída e um sinal desejado. Para tanto, o erro é alimentado de volta para o filtro, cujos coeficientes são atualizados através de um algoritmo adaptativo.

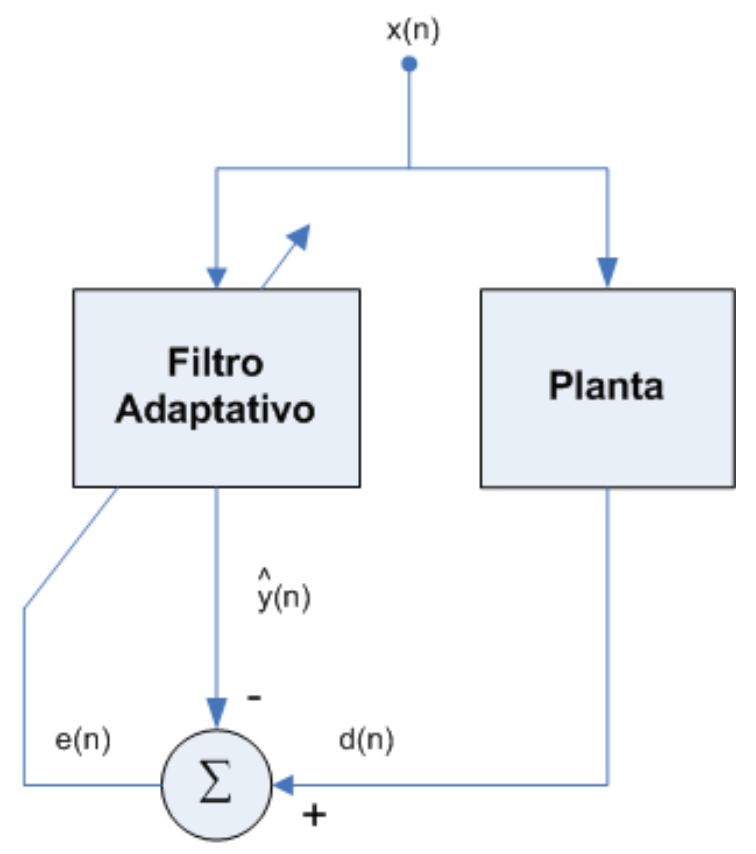

Figura 4: Diagrama de um Sistema Adaptativo para identificação de sistemas.

No caso do cancelamento de eco acústico através de um sistema representado pelo diagrama da Figura 4, no qual o sinal desejado é igual ao sinal de eco acústico, a saída ideal do filtro adaptativo seria igual ao valor do sinal de eco indesejado, fazendo com que o sinal de erro resultasse em zero. Nesta situação, o sinal de eco seria completamente cancelado e o usuário não ouviria a repetição do sinal sonoro emitido por ele mesmo.

\subsubsection{Filtro FIR Transversal}

Um filtro pode ser definido como uma implementação de software ou hardware que recebe um sinal de entrada e o processa a fim de extrair e evidenciar certos elementos desejados do sinal [29]. 
Dada uma sequência de entrada discreta de números reais $x(n)$, onde $n$ é um valor de índice inteiro, a sequência de saída $y(n)$ de um filtro FIR variante no tempo de ordem $N-1$ é definida pela equação (2.1):

$$
y(n)=\sum_{i=0}^{N-1} w_{i}(n) x(n-i)
$$

onde o valor do índice $n$ representa o instante de tempo discreto atual; $n-i$ representa o $i$-ésimo instante anterior, ou seja, atrasado de $i$ instantes; e $w_{i}(n)$ são os coeficientes que definem as características do filtro.

De fato, para implementar um filtro FIR a equação (2.1) deve ser convertida em blocos realizáveis, incluindo um meio para a obtenção de uma versão atrasada da sequência de entrada $\mathbf{x}(n)$, um meio para a multiplicação de valores de sinal de entrada pelos coeficientes do filtro, w, e um meio para adicionar os valores intermediários da sequência de saída $\mathbf{y}(n)$. A estrutura transversal apresentada na Figura 5 apresenta uma maneira direta de realização do filtro FIR, o qual estará completamente definido uma vez que os coeficientes $\mathbf{w}$ do filtro forem conhecidos [30] .

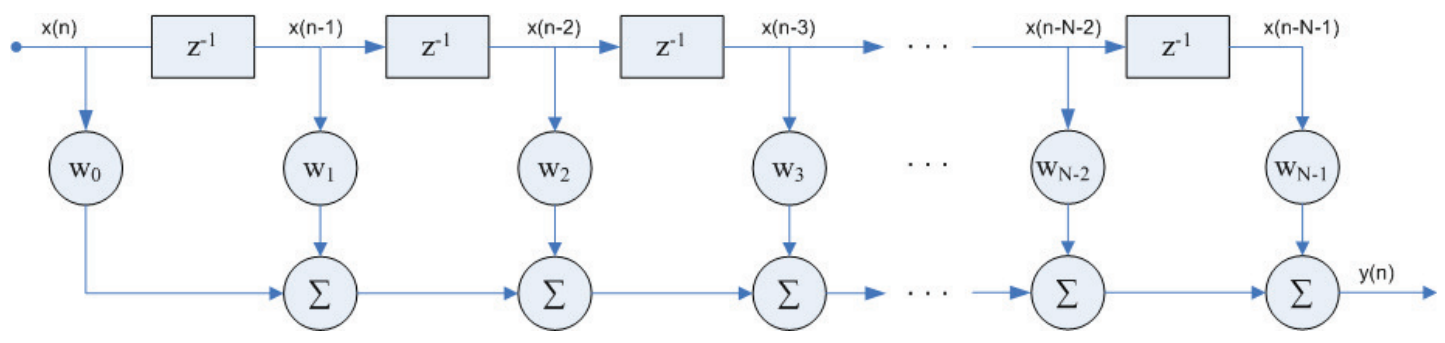

Figura 5: Estrutura FIR Transversal.

\subsubsection{Filtro de Wiener}

Filtros de Wiener FIR têm seus coeficientes calculados como segue. Inicialmente podemos reescrever em forma vetorial a saída de um filtro FIR de ordem 
$N-1$ como:

$$
y(n)=\sum_{i=0}^{N-1} w_{i}(n) x(n-i)=\mathbf{w}^{T}(n) \mathbf{x}(n)
$$

onde $\mathbf{x}(n)=[x(n) x(n-1) \ldots x(n-N+1)]^{T}$ e $\mathbf{w}(n)=\left[w_{0}(n) w_{1}(n) \ldots w_{N-1}(n)\right]^{T}$ são, respectivamente, vetores de entrada e dos parâmetros do filtro.

Com o sinal de erro

$$
e(n)=d(n)-y(n)=d(n)-\mathbf{w}^{T}(n) \mathbf{x}(n)
$$

e definindo a função custo como sendo o erro médio quadrático, obtém-se

$$
\begin{gathered}
\xi(n)=E\left[e^{2}(n)\right] \\
\xi(n)=E\left[d^{2}(n)-2 d(n) y(n)+y^{2}(n)\right] \\
\xi(n)=E\left[d^{2}(n)-2 d(n) \mathbf{w}^{T}(n) \mathbf{x}(n)+\mathbf{w}^{T}(n) \mathbf{x}(n) \mathbf{x}^{T}(n) \mathbf{w}(n)\right] \\
\xi(n)=E\left[d^{2}(n)\right]-2 E\left[d(n) \mathbf{w}^{T}(n) \mathbf{x}(n)\right]+E\left[\mathbf{w}^{T}(n) \mathbf{x}(n) \mathbf{x}^{T}(n) \mathbf{w}(n)\right]
\end{gathered}
$$

Considerando que os coeficientes do filtro de Wiener são constantes, a função custo é dada por:

$$
\begin{gathered}
\xi(n)=E\left[d^{2}(n)\right]-2 \mathbf{w}^{T} E[d(n) \mathbf{x}(n)]+\mathbf{w}^{T} E\left[\mathbf{x}(n) \mathbf{x}^{T}(n)\right] \mathbf{w} \\
\xi(n)=E\left[d^{2}(n)\right]-2 \mathbf{w}^{T} \mathbf{p}+\mathbf{w}^{T} \mathbf{R} \mathbf{w}
\end{gathered}
$$

onde $\mathbf{p}=E[d(n) \mathbf{x}(n)]$ é o vetor de correlação cruzada entre o sinal desejado e o sinal de entrada, e $\mathbf{R}=E\left[\mathbf{x}(n) \mathbf{x}^{T}(n)\right]$ é a matriz de auto-correlação do sinal de entrada.

A função custo acima é uma função quadrática $N$-dimensional e sua minimização leva a uma solução ótima única, obtendo um vetor dos coeficientes ótimos, $\mathbf{w}_{0}$.

O valor mínimo de $\xi(n)$ pode ser encontrado através do cálculo do seu vetor 
gradiente relacionado aos coeficientes do filtro:

$$
\frac{\partial \xi}{\partial \mathbf{w}}=\left[\frac{\partial \xi}{\partial w_{0}} \frac{\partial \xi}{\partial w_{1}} \ldots \frac{\partial \xi}{\partial w_{N}}\right]^{T}=-2 \mathbf{p}+2 \mathbf{R} \mathbf{w}
$$

Assumindo que $\mathbf{R}$ é uma matriz não singular e igualando-se a zero a equação anterior, obtêm-se a solução de Wiener ótima para os coeficientes do filtro, $\mathbf{w}_{0}$.

$$
\mathbf{w}_{0}=\mathbf{R}^{-1} \mathbf{p}
$$

\subsubsection{Filtro Adaptativo Transversal}

Um sistema de filtro adaptativo filtra uma sequência de dados de entrada, controlando seus parâmetros ajustáveis através de um processo adaptativo. A escolha da estrutura de filtragem é uma parte muito importante do sistema e a estrutura transversal é utilizada com sucesso em muitos sistemas práticos.

A realização de um filtro adaptativo transversal usando elementos computacionais básicos é mostrada na Figura 6, sendo que os taps são representados como blocos $z^{-1}$ significando um atraso de uma unidade do instante de tempo $i$. Observe que a sequência de entrada $\mathbf{x}(n)$ flui através da linha de atraso de forma contínua e uniforme produzindo um outro valor de saída $\mathbf{y}(n)$ a cada passo, indefinidamente. Este filtro pode ser transformado em um filtro adaptativo por meio da adição de um mecanismo de adaptação adequado que é capaz de adaptar os coeficientes $\mathbf{w}$ progressivamente a cada passo, baseado em alguma informação de dado de tempo real.

\subsubsection{Algoritmos Adaptativos Lineares}

O algoritmo LMS é o mais utilizado dentre os algoritmos de gradiente estocásticos, por isso a maioria dos canceladores de eco usam variantes do algoritmo LMS. 


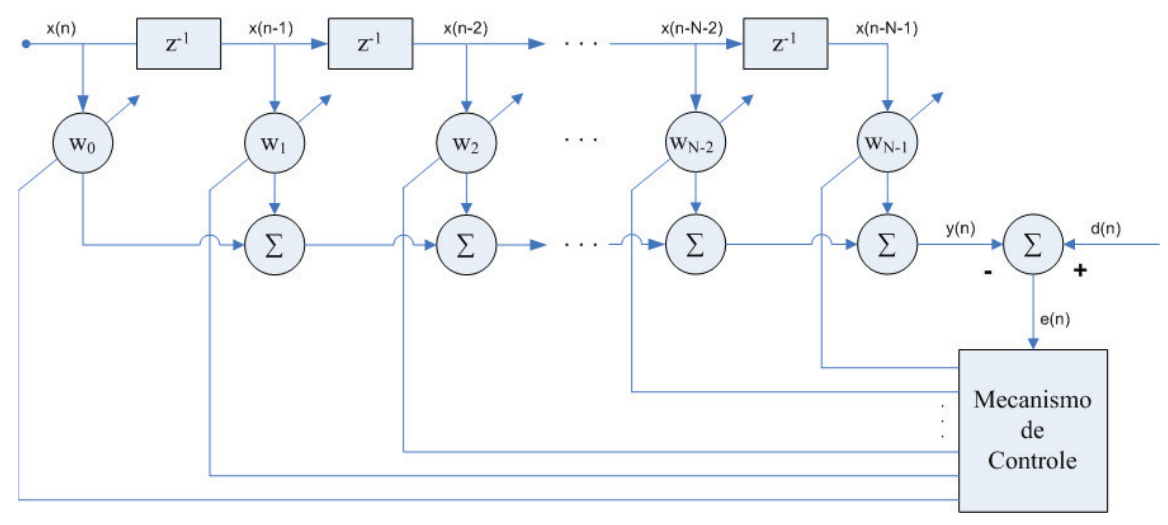

Figura 6: Filtro Adaptativo Transversal.

A vantagem do algoritmo LMS é sua facilidade de implementação devido à necessidade relativamente baixa de memória e poucos requisitos computacionais. A principal desvantagem do algoritmo LMS é o fato de que sua taxa de convergência é sensível ao espalhamento dos auto-valores do sinal de entrada.

Nessa subseção serão descritos os algoritmos adaptativos lineares utilizados no presente trabalho. Em seguida, o próximo capítulo é dedicado às estruturas não-lineares a serem utilizadas no sistema cancelador de eco acústico proposto no Capítulo 5.

\subsubsection{Least Mean Square - LMS}

O algoritmo LMS é baseado no gradiente do erro quadrático médio. A cada iteração, os coeficientes do filtro adaptativo são atualizados de acordo com a seguinte fórmula [29]:

$$
\mathbf{w}(n+1)=\mathbf{w}(n)+\mu e(n) \mathbf{x}(n)
$$

onde $\mathbf{x}(n)=[x(n) x(n-1) x(n-2) \ldots x(n-N+1)]^{T}$ é o vetor de amostras de entrada; e o vetor $\mathbf{w}(n)=\left[w_{0}(n) w_{1}(n) w_{2}(n) \ldots w_{N-1}(n)\right]^{T}$ reúne os coeficientes do filtro FIR adaptativo no tempo $n$.

O parâmetro $\mu$ é o passo de adaptação do algoritmo e deve ser uma constante 
positiva pequena em relação à potência do sinal de entrada. A seleção de um valor adequado para $\mu$ é determinante para o desempenho do algoritmo LMS: se o valor for pequeno demais o tempo que o filtro adaptativo leva para convergir para a solução ótima será muito longo; se $\mu$ é muito grande o filtro adaptativo torna-se instável e sua saída diverge.

A derivação do algoritmo LMS baseia-se na teoria da solução ótima de Wiener e também depende do algoritmo de gradiente descendente [31], que atualiza os coeficientes do filtro usando o vetor de coeficientes atual e o gradiente atual da função custo em relação ao vetor de coeficientes do filtro $\Delta_{w}(n)$, de acordo com

$$
\mathbf{w}(n+1)=\mathbf{w}(n)-\frac{1}{2} \mu \Delta_{w}(n) .
$$

Considerando que

$$
\Delta_{w}(n)=\frac{\partial \xi}{\partial \mathbf{w}}
$$

e substituindo (2.6) na equação (2.9), temos:

$$
\mathbf{w}(n+1)=\mathbf{w}(n)+\mu(\mathbf{p}(n)-\mathbf{R}(n) \mathbf{w}(n))
$$

A equação (2.11) usa o gradiente do erro médio quadrático, que também é desconhecido. A matriz de auto-correlação $\mathbf{R}$ e o vetor de correlação cruzada $\mathbf{p}$, são estimados da seguinte forma:

$$
\begin{aligned}
\hat{\mathbf{R}}(n) & =\mathbf{x}(n) \mathbf{x}^{T}(n) \\
\hat{\mathbf{p}}(n) & =d(n) \mathbf{x}(n)
\end{aligned}
$$

sendo que

$$
\begin{aligned}
& E[\hat{\mathbf{R}}]=\mathbf{R} \\
& E[\hat{\mathbf{p}}]=\mathbf{p}
\end{aligned}
$$


Resulta a seguinte estimativa instantânea do gradiente:

$$
\begin{aligned}
\hat{\Delta}_{w}(n) & \left.=-d(n) \mathbf{x}(n)+\mathbf{x}(n) \mathbf{x}^{T}(n)\right) \mathbf{w}(n) \\
& =\mathbf{x}(n)\left(-d(n)+\mathbf{x}^{T}(n) \mathbf{w}(n)\right)
\end{aligned}
$$

E, observando-se que,

$$
e(n)=d(n)-\mathbf{w}^{T}(n) \mathbf{x}(n)
$$

Essa estimativa se torna:

$$
\hat{\Delta}_{w}(n)=-e(n) \mathbf{x}(n)
$$

Resultando na equação de atualização dos coeficientes do filtro adaptativo:

$$
\mathbf{w}(n+1)=\mathbf{w}(n)+\mu e(n) \mathbf{x}(n)
$$

Essa equação recursiva resultante define o algoritmo LMS; e os passos para sua implementação podem ser evidenciados de forma sequencial na Tabela 1:

\begin{tabular}{|c||}
\hline Algoritmo LMS \\
Inicialização \\
$\mathbf{x}(0)=\mathbf{w}(0)=\left[\begin{array}{llll}0 & 0 & \ldots & 0\end{array}\right]^{T}$ \\
Faça para $\mathrm{n} \geqslant 0$ \\
$e(n)=d(n)-\mathbf{w}^{T}(n) \mathbf{x}(n)$ \\
$\mathbf{w}(n+1)=\mathbf{w}(n)+\mu e(n) \mathbf{x}(n)$
\end{tabular}

Tabela 1: Etapas de implementação do algoritmo LMS [29]. 


\subsubsection{Normalized Least Mean Square - NLMS}

Admitindo que o sinal de erro $e(n)$ tem uma dependência a grosso modo linear com o sinal de entrada $\mathrm{x}(\mathrm{n})$, na forma padrão do algoritmo LMS a correção $\mu \mathbf{x}(n) e(n)$ tem uma dependência a grosso modo quadrática com a entrada. Desta forma, ao escolher um valor adequado para o passo de adaptação a potência do sinal de entrada deve ser levada em conta. Para contornar este inconveniente, podemos usar o algoritmo NLMS, cujo fator de correção dos coeficientes $w(n)$ na iteração $n+1$ é normalizado com relação ao quadrado da norma Euclidiana do vetor $\mathbf{x}(n)$ na iteração $n$ [31].

A derivação do algoritmo NLMS pode ser realizada modificando-se o algoritmo LMS de uma forma natural fazendo uso de um passo de adaptação variável $\mu_{k}$ a fim de aumentar a velocidade de convergência. Assim, a equação de atualização do algoritmo LMS pode ser expressa como:

$$
\mathbf{w}(n+1)=\mathbf{w}(n)+\mu_{k} e(n) \mathbf{x}(n)
$$

onde $\mu_{k}$ é o passo de adaptação variável.

Retomando a expressão do erro médio quadrático apresentado na derivação da solução de Wiener ótima, o erro quadrático instantâneo é dado por:

$$
e^{2}(n)=d^{2}(n)+\mathbf{w}^{T}(n) \mathbf{x}(n) \mathbf{x}^{T}(n) \mathbf{w}(n)-2 d(n) \mathbf{w}^{T}(n) \mathbf{x}(n)
$$

Alterando o vetor dos coeficientes de tal forma que:

$$
\mathbf{w}^{\prime}(n)=\mathbf{w}(n)+\Delta \mathbf{w}^{\prime}(n)
$$

O erro quadrático torna-se:

$$
\begin{aligned}
e^{\prime 2}(n)= & e^{2}(n)+2 \Delta \mathbf{w}^{\prime T}(n) \mathbf{x}(n) \mathbf{x}^{T}(n) \mathbf{w}(n) \\
& +\Delta \mathbf{w}^{\prime T}(n) \mathbf{x}(n) \mathbf{x}^{T}(n) \Delta \mathbf{w}^{\prime}(n)-2 d(n) \Delta \mathbf{w}^{\prime T}(n) \mathbf{x}(n)
\end{aligned}
$$


E segue que:

$$
\begin{gathered}
\Delta e^{2}(n) \cong e^{\prime 2}(n)-e^{2}(n) \\
\Delta e^{2}(n)=2 \Delta \mathbf{w}^{\prime T}(n) \mathbf{x}(n) \mathbf{x}^{T}(n) \mathbf{w}(n) \\
+\Delta \mathbf{w}^{\prime T}(n) \mathbf{x}(n) \mathbf{x}^{T}(n) \Delta \mathbf{w}^{\prime}(n)-2 d(n) \Delta \mathbf{w}^{\prime T}(n) \mathbf{x}(n)
\end{gathered}
$$

Considerando:

$$
e(n)=d(n)-\mathbf{x}^{T}(n) \mathbf{w}(n)
$$

A expressão de $\Delta e^{2}(n)$ pode ser escrita da seguinte forma:

$$
\Delta e^{2}(n)=-2 \Delta \mathbf{w}^{\prime T}(n) \mathbf{x}(n) e(n)+\Delta \mathbf{w}^{\prime T}(n) \mathbf{x}(n) \mathbf{x}^{T}(n) \Delta \mathbf{w}^{\prime}(n)
$$

Como:

$$
\Delta \mathbf{w}^{\prime}(n)=\mu_{k} e(n) \mathbf{x}(n)
$$

Substituindo $\Delta \mathbf{w}^{\prime}(n)$ na expressão de $\Delta e^{2}(n)$, obtém-se:

$$
\Delta e^{2}(n)=-2 \mu_{k} e^{2}(n) \mathbf{x}^{T}(n) \mathbf{x}(n)+\mu_{k}^{2} e^{2}(n)\left[\mathbf{x}(n) \mathbf{x}^{T}(n)\right]^{2}
$$

Diferenciando o erro quadrático com relação à $\mu_{k}$ e igualando a zero:

$$
\frac{\partial \Delta e^{2}(n)}{\partial \mu_{k}}=0
$$

Obtemos,

$$
\mu_{k}=\frac{1}{\mathbf{x}^{T}(n) \mathbf{x}(n)}
$$

Substituindo o valor encontrado de $\mu_{k}$ na expressão de $\Delta e^{2}(n)$ obtém-se um valor negativo. Portanto, $\mu_{k}$ corresponde a um ponto mínimo de $\Delta e^{2}(n)$.

Assim sendo, a equação de atualização torna-se:

$$
\mathbf{w}(n+1)=\mathbf{w}(n)+\frac{\mathbf{x}(n)}{\mathbf{x}^{T}(n) \mathbf{x}(n)}
$$


Um passo de adaptação fixo $\mu_{f}$ é introduzido na equação de atualização para para suavizar a adaptação, já que a derivação da equação foi baseada em valores instantâneos dos erros quadráticos e não dos erros médios quadráticos. Um parâmetro $\gamma$ também é introduzido para prevenir um valor muito elevado do passo de atualização, quando $\mathbf{x}^{T}(n) \mathbf{x}(n)$ torna-se muito pequeno. Finalmente, chega-se a forma final da equação de atualização dos coeficientes do filtro adaptativo:

$$
\mathbf{w}(n+1)=\mathbf{w}(n)+\frac{\mu_{f}}{\gamma+\mathbf{x}^{T}(n) \mathbf{x}(n)} e(n) \mathbf{x}(n)
$$

Essa equação de atualização resultante define o algoritmo NLMS; e os passos para sua implementação podem ser evidenciados de forma sequencial na Tabela 2:

\begin{tabular}{c}
\hline Algoritmo NLMS \\
Inicialização \\
$\mathbf{x}(0)=\mathbf{w}(0)=\left[\begin{array}{llll}0 & 0 & \ldots & 0\end{array}\right]^{T}$ \\
$\gamma \ll \frac{N}{\sigma^{2}}$ \\
de modo que em média \\
$\gamma \ll E\left[\mathbf{x}^{T}(n) \mathbf{x}(n)\right]$ \\
Faça para $\mathbf{n} \geqslant 0$ \\
$e(n)=d(n)-\mathbf{x}^{T}(n) \mathbf{w}(n)$ \\
$\mathbf{w}(n+1)=\mathbf{w}(n)+\frac{\mu_{f}}{\gamma+\mathbf{x}^{T}(n) \mathbf{x}(n)} e(n) \mathbf{x}(n)$
\end{tabular}

Tabela 2: Etapas de implementação do algoritmo NLMS [29].

Importante destacar o fato de ser uma alternativa ao algoritmo clássico LMS, o algoritmo NLMS lida com uma função de custo do erro médio quadrático modificada, assim a solução ideal esperada pode diferir da solução de Wiener [32]. 


\subsubsection{Affine Projection Algorithm - APA}

O algoritmo APA é uma generalização do algoritmo NLMS. Neste último caso, cada atualização do vector de coeficientes do filtro pode ser visto como uma projeção afim unidimensional. O Algoritmo APA generaliza este conceito, permitindo que as projeções possam ser feitas em múltiplas dimensões. A medida que a dimensão da projeção aumenta, o mesmo acontece com a velocidade de convergência do vetor de coeficientes.

O algoritmo APA é apresentado na Tabela, com todas as etapas para sua implementação listadas sequencialmente:

\begin{tabular}{|c||}
\hline Algoritmo APA \\
Inicialização \\
$\mathbf{x}(0)=\mathbf{w}(0)=\left[\begin{array}{llll}0 & 0 & \ldots & 0\end{array}\right]^{T}$ \\
$\gamma \ll 1$ \\
$0 \leqslant \mu \leqslant 2$ \\
Faça para $\mathrm{n} \geqslant 0$ \\
$\mathbf{e}_{a p}(n)=\mathbf{d}_{a p}(n)-\mathbf{X}_{a p}^{T}(n) \mathbf{w}(n)$ \\
$\mathbf{w}(n+1)=\mathbf{w}(n)+\mu \mathbf{X}_{a p}(n)\left(\gamma \mathbf{I}+\mathbf{X}_{a p}^{T}(n) \mathbf{X}_{a p}(n)\right)^{-1} \mathbf{e}_{a p}(n)$ \\
\end{tabular}

Tabela 3: Etapas de implementação do algoritmo APA [29]. 
A matriz do sinal de excitação $\mathbf{X}_{a p}(n)$ e o vetor do sinal desejado $\mathbf{d}_{a p}(n)$ são definidos como:

$$
\begin{aligned}
\mathbf{X}_{a p}(n) & =\left[\begin{array}{llll}
\mathbf{x}(n) & \mathbf{x}(n-1) & \ldots & \mathbf{x}(n-p+1)
\end{array}\right]^{T} \\
\mathbf{d}_{a p}(n) & =\left[\begin{array}{llll}
d(n) & d(n-1) & \ldots & d(n-p+1)
\end{array}\right]^{T}
\end{aligned}
$$

Uma vez que $\mathbf{X}_{a p}(n) \mathbf{X}_{a p}^{T}(n)$ pode ter valores perto de zero (criando problemas ao calcular sua inversa), um pequeno parâmetro $\gamma$ e uma pequena matriz diagonal $\mathbf{I}$ são adicionados à $\mathbf{X}_{a p}(n) \mathbf{X}_{a p}^{T}(n)$ antes da inversão. $\gamma$ é uma pequena constante positiva denominada parâmetro de regularização para a matriz de autocorrelação do sinal de excitação $\mathbf{X}_{a p}(n) \mathbf{X}_{a p}^{T}(n)$. O parâmetro $\gamma$ deve ser tão pequeno quanto possível, enquanto ainda permitindo que a inversa seja bem-condicionada.

A constante $\mu$ define o tamanho do passo e é, às vezes, chamado de fator de relaxamento do algoritmo APA. O parâmetro $p$, chamado de ordem da projeção, define a dimensão dos vetores $\mathbf{d}_{a p}, \mathbf{e}_{a p}$ e da matriz $\mathbf{X}_{a p}$. Se $p$ é definido como 1 , então o algoritmo APA se reduz ao algoritmo NLMS.

Há pouca diferença entre o comportamento de convergência dos algoritmos NLMS e APA utilizando excitação de ruído branco. No entanto, com sinais de excitação coloridos, o algoritmo APA pode ter uma taxa de convergência muito mais rápida do que a taxa de convergência do algoritmo NLMS [1], especialmente quando a ordem de projeção $p$ é aumentada. Logo, o algoritmo APA é muito atraente para cancelamento de eco acústico [21], já que possui uma característica de convergência mais eficiente do que o algoritmo NLMS quando se usam sinais de fala como entrada do filtro adaptativo [27]. 


\subsubsection{Fast Block Least Mean Square - FBLMS}

Nos algoritmos considerados até aqui a adaptação ocorre no domínio do tempo. Uma vez que a transformada de Fourier mapeia os sinais no domínio do tempo para o domínio da frequência e a transformada de Fourier inversa fornece o mapeamento inverso, trazendo os sinais de volta para o domínio do tempo, é viável realizar a adaptação dos coeficientes do filtro no domínio da frequência, o que é chamado filtragem adaptativa no domínio da frequência [31].

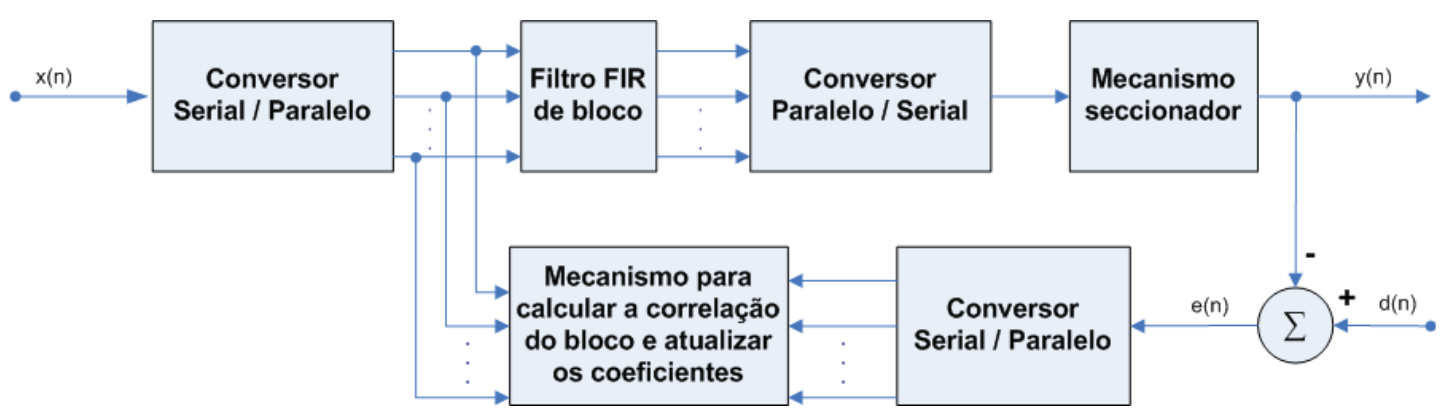

Figura 7: Filtro adaptativo em Bloco.

Em um filtro adaptativo em bloco, mostrado na Figura 7, a sequência de dados de entrada $x(n)$ é seccionada em blocos de $M$ pontos por meio de um conversor série-paralelo, e os blocos de dados de entrada, assim produzidos, são aplicados a um filtro FIR de comprimento $M$, um bloco por vez. Os coeficientes do filtro são atualizados após a coleta de cada bloco de amostras de dados, de modo que a adaptação dos filtros procede numa base bloco-por-bloco, ao invés de proceder numa base amostra-por-amostra como no algoritmo LMS convencional.

O algoritmo FBLMS representa uma implementação no domínio da frequência do algoritmo LMS de bloco e suas propriedades de convergência são idênticas às do algoritmo de LMS de bloco, o qual, por sua vez, apresenta propriedades de convergência similares ao do algoritmo LMS convencional [31].

O algoritmo FBLMS é apresentado na Tabela 4, com todas as etapas para sua implementação listadas sequencialmente. 


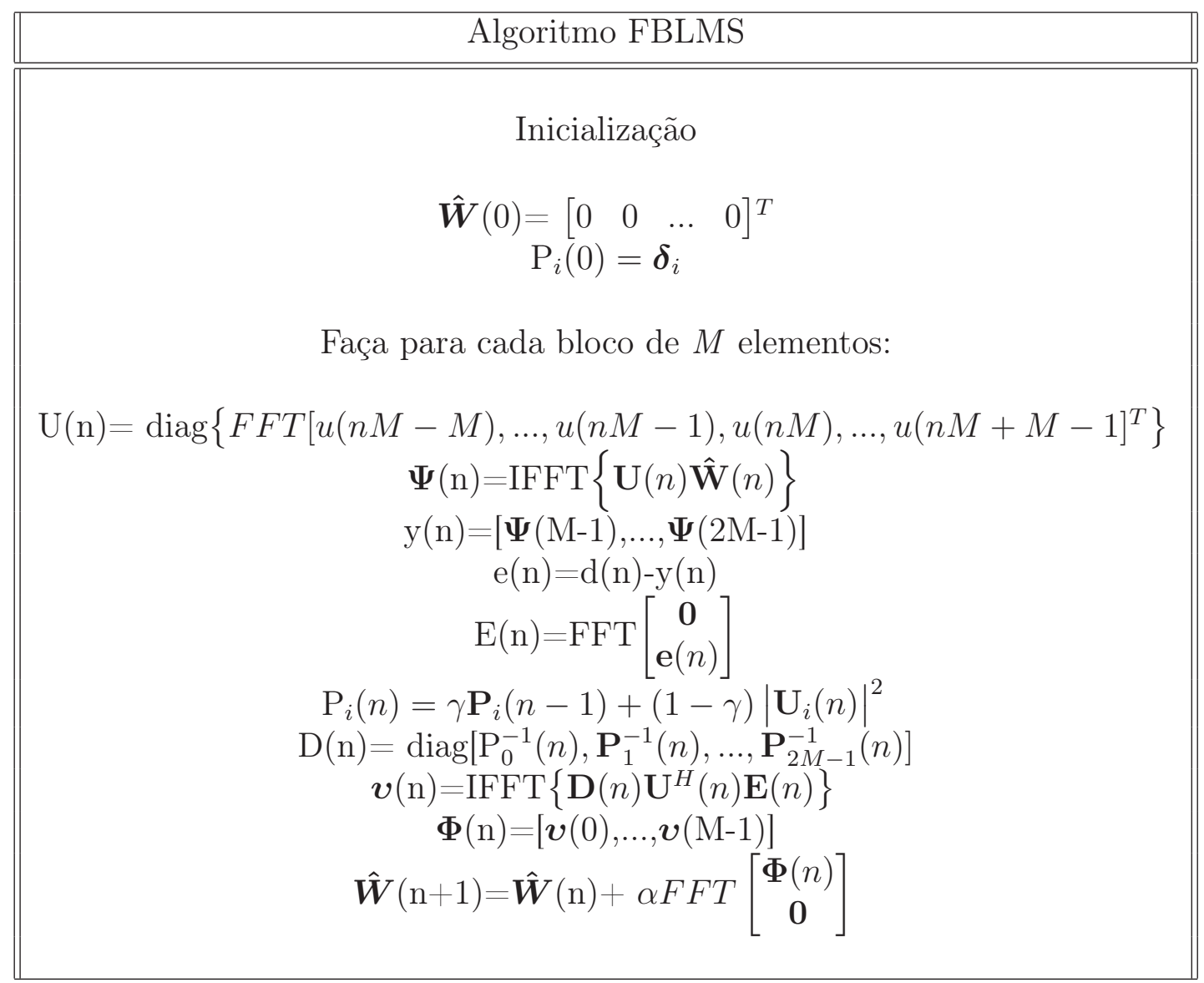

Tabela 4: Etapas de implementação do algoritmo FBLMS [29]. 


\section{ESTRUTURAS NÃO-LINEARES}

Esse trabalho emprega o modelo de parâmetros passivos para alto-falantes eletrodinâmicos proposto em [19] como bloco de pré-processamento não-linear e como modelo do alto-falante no sistema cancelador de eco acústico proposto no Capítulo 5 .

Um alto-falante automotivo para graves foi modelado em [19], sendo que conceitos relacionados à alto-falantes dinâmicos são apresentados brevemente nesse capítulo, assim como as equações de espaço de estados e valores de parâmetros do modelo. Adicionalmente, um estudo sobre a precisão do modelo realizado em [18] também será mencionado.

As estruturas não-lineares a serem utilizadas como bloco NLP serão implementadas através de um conjunto de scripts do Matlab previamente desenvolvidos e consolidados na forma de um toolbox computacional para fins de implementação de modelos não-lineares, baseados em núcleos de Volterra e bilinearização de Carleman, que representam sistemas físicos reais [33].

\subsection{Modelo de Parâmetros Passivos para Alto- falantes Eletrodinâmicos}

O modelo de parâmetros passivos se mostrou preciso na representação do comportamento de um alto-falante eletrodinâmico real conforme os resultados apresentados em [19], por isso adotou-se esse modelo no presente trabalho. 


\subsubsection{Alto-falantes Dinâmicos}

O alto-falante é um transdutor eletroacústico que converte um sinal elétrico em um sinal acústico. As principais partes do alto-falante dinâmico são a bobina de voz, a peça polar, o imã, o diafragma, a aranha, a tampa de poeira, o aro, a placa superior e a armação, conforme ilustrado na Figura 8.

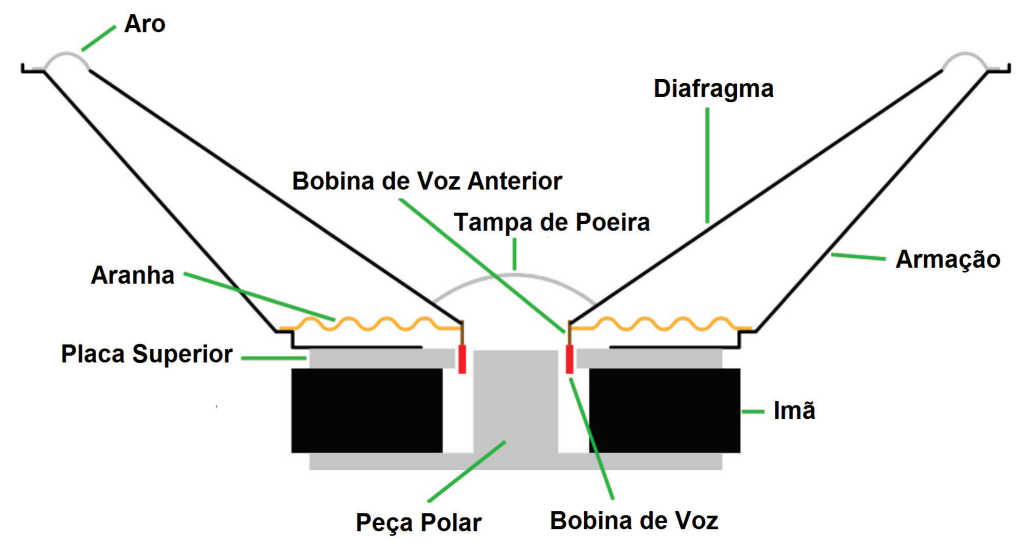

Figura 8: Esquema seccional de um alto-falante eletrodinâmico. Adaptado de [34].

Quando o alto-falante recebe um sinal elétrico, a corrente flui na bobina de voz e a sua interação com o campo magnético do ímã permanente dá origem a uma força. Essa força provoca o deslocamento da bobina de voz, que é então transferido para o diafragma e este irradia som.

\subsubsection{Equações de espaço de estados}

No apêndice F serão apresentadas breves descrições dos conceitos envolvidos na modelagem do alto-falante e dedução das equações de espaço de estados do modelo, as quais são apresentadas a seguir. 
Considerando o vetor de estados como

$$
\boldsymbol{x}=\left[\begin{array}{l}
x \\
\dot{x} \\
i
\end{array}\right]=\left[\begin{array}{l}
x_{1} \\
x_{2} \\
x_{3}
\end{array}\right]
$$

a representação do modelo em equações de espaço de estados é dada por

$$
\begin{gathered}
\dot{\boldsymbol{x}}=\left[\begin{array}{c}
x_{2} \\
\frac{1}{M}\left\{a\left(x_{1}\right) x_{3}+\frac{x_{3}^{2}}{2} L^{\prime}\left(x_{1}\right)-k\left(x_{1}\right) x_{1}-G x_{2}\right\} \\
-\frac{1}{L\left(x_{1}\right)}\left\{R x_{3}+L^{\prime}\left(x_{1}\right) x_{2} x_{3}+a\left(x_{1}\right) x_{2}\right\}
\end{array}\right]+\left[\begin{array}{c}
0 \\
0 \\
\frac{1}{L\left(x_{1}\right)}
\end{array}\right] e \\
y=\left[\begin{array}{lll}
1 & 0 & 0
\end{array}\right] \boldsymbol{x}
\end{gathered}
$$

As funções $a\left(x_{1}\right), k\left(x_{1}\right)$ e $L\left(x_{1}\right)$ que modelam o comportamento do fator de força, da rigidez da suspensão do diafragma e da indutância não-linear da bobina de voz são descritas abaixo, conforme [19].

$$
\begin{gathered}
a\left(x_{1}\right)=\frac{a_{0}}{1+a_{1}\left(x_{1}-x_{a}\right)^{2}} \\
k\left(x_{1}\right)=k_{0}+k_{1}\left(x_{1}-x_{k}\right)^{2} \\
L\left(x_{1}\right)=l_{0}+l_{1}\left(1-\frac{x_{1}-x_{l}}{l_{2}+\left\|x_{1}-x_{l}\right\|}\right)
\end{gathered}
$$

As equações aproximadas de $a\left(x_{1}\right)$ e $L\left(x_{1}\right)$ são derivadas em [19] através da inspeção do comportamento dos componentes do alto-falante eletrodinâmico nos limites de deslocamento do diafragma. Por sua vez, a equação aproximada de $k\left(x_{1}\right)$ é originalmente proposta em [35]. 


\subsubsection{Precisão do Modelo}

Um alto-falante automotivo para gravez com diâmetro de $82 \mathrm{~mm}$ foi modelado em [19], sendo que as equações de espaço de estados apresentadas na seção anterior foram empregadas em tal tarefa. Inicialmente, a fim de se obter uma estimativa dos parâmetros que compõem o modelo, os autores do artigo fizeram uma análise para pequenos sinais do alto-falante e, em seguida, executaram um processo de adaptação, onde o objetivo era a minimização do erro entre a corrente medida e aquela fornecida pelo modelo. Com isso, foram obtidos os valores mostrados na Figura 9 .

\begin{tabular}{ccc}
\hline \multicolumn{3}{c}{ Função de rigidez $k\left(x_{1}\right)$} \\
\hline Parâmetro & Unidade & Valores \\
\hline$k_{0}$ & $\mathrm{~N} / \mathrm{m}$ & $6,753 \times 10^{2}$ \\
$k_{1}$ & $\mathrm{~N} / \mathrm{m}^{3}$ & $6,75 \times 10^{4}$ \\
$x_{k}$ & $\mathrm{~m}$ & $7,618 \times 10^{-4}$ \\
\hline
\end{tabular}

\begin{tabular}{ccc}
\hline \multicolumn{3}{c}{ Função de indutância $L\left(x_{1}\right)$} \\
\hline Parâmetro & Unidade & Valores \\
\hline$l_{0}$ & $\mathrm{H}$ & $1,22 \times 10^{-5}$ \\
$l_{1}$ & $\mathrm{H}$ & $2,309 \times 10^{-4}$ \\
$l_{2}$ & $\mathrm{~m}$ & $3 \times 10^{-3}$ \\
$x_{l}$ & $\mathrm{~m}$ & $-1 \times 10^{-3}$ \\
\hline
\end{tabular}

\begin{tabular}{ccc}
\hline \multicolumn{3}{c}{ Função do fator de força $A\left(x_{1}\right)$} \\
\hline Parâmetro & Unidade & Valores \\
\hline$a_{0}$ & N/A & 2,6607 \\
$a_{1}$ & $1 / \mathrm{m}^{2}$ & $9,2 \times 10^{4}$ \\
$x_{a}$ & $\mathrm{~m}$ & $-3,463 \times 10^{-4}$ \\
\hline
\end{tabular}

\begin{tabular}{ccc}
\hline \multicolumn{3}{c}{ Características dos componentes } \\
\hline Parâmetro & Unidade & Valores \\
\hline$M$ & $\mathrm{~kg}$ & $3 \times 10^{-3}$ \\
$G$ & $\mathrm{Ns} / \mathrm{m}$ & 0,8319 \\
$R$ & $\Omega$ & 4,6254 \\
\hline
\end{tabular}

Figura 9: Valores numéricos dos parâmetros do modelo do alto-falante. Extraído de [19].

Contudo, foi necessário formular o modelo de forma aproximada conforme realizado em [18], já que originalmente existe uma função não-analítica envolvida em suas equações de estado, ao passo que as equações fornecidas como entrada do algoritmo de bilinearização de Carleman devem ser analíticas. Assim, um ajuste foi feito no modelo através da substituição da função $L\left(x_{1}\right)$ por uma aproximação 
na forma

$$
\widetilde{L}\left(x_{1}\right)=b_{0}+b_{1} \tan ^{-1}\left(w_{l} x_{1}+\phi_{l}\right)
$$

Usando um algoritmo de otimização não-linear e o critério de mínimos quadrados, os parâmetros da equação acima foram estimados em [18], resultando nos valores mostrados na Figura 10.

\begin{tabular}{ccc}
\hline Parâmetro & Valor estimado & Limites de confiança de $95 \%$ \\
\hline$b_{0}$ & $0,2423 \times 10^{-4}$ & $\left(0,2421 \times 10^{-4}, 0,2425 \times 10^{-4}\right)$ \\
$b_{1}$ & $-0,1303 \times 10^{-4}$ & $\left(-0,1300 \times 10^{-4},-0,1306 \times 10^{-4}\right)$ \\
$\omega_{l}$ & 419,2 & $(416,7,421,7)$ \\
$\phi_{l}$ & 0,4089 & $(0,4050,0,4128)$ \\
\hline
\end{tabular}

Figura 10: Valores estimados para a aproximação de $L\left(x_{1}\right)$. Extraído de [18].

A precisão do modelo foi verificada fazendo uma comparação entre o sinal de saída do alto-falante real e aquele proveniente do modelo físico aqui descrito, quando ambos foram excitados por um sinal de entrada gerado sobre certas condições de interesse.

A energia desse sinal de entrada foi concentrada em torno dos $100 \mathrm{~Hz}$, a frequência de ressonância do alto-falante, para que os efeitos das não-linearidades fossem intensificados. Além disso, a faixa de frequências do sinal de entrada foi limitada entre $20 \mathrm{~Hz}$ e $500 \mathrm{~Hz}$, pois o alto-falante em questão é um Woofer, e por consequência, possui um comportamente de filtro passa-baixas com frequência de corte em $500 H z$.

O gráfico dos sinais de saída é apresentado na Figura (11) e observando a proximidade entre as linhas sólida e tracejada, saída do alto-falante real e modelo passivo, respectivamente, pode-se notar que o modelo é capaz de representar o comportamento do alto-falante real com precisão. Importante mencionar que o erro quadrático médio obtido com o uso do modelo não é citado em [19], apenas 
Nonlinear model

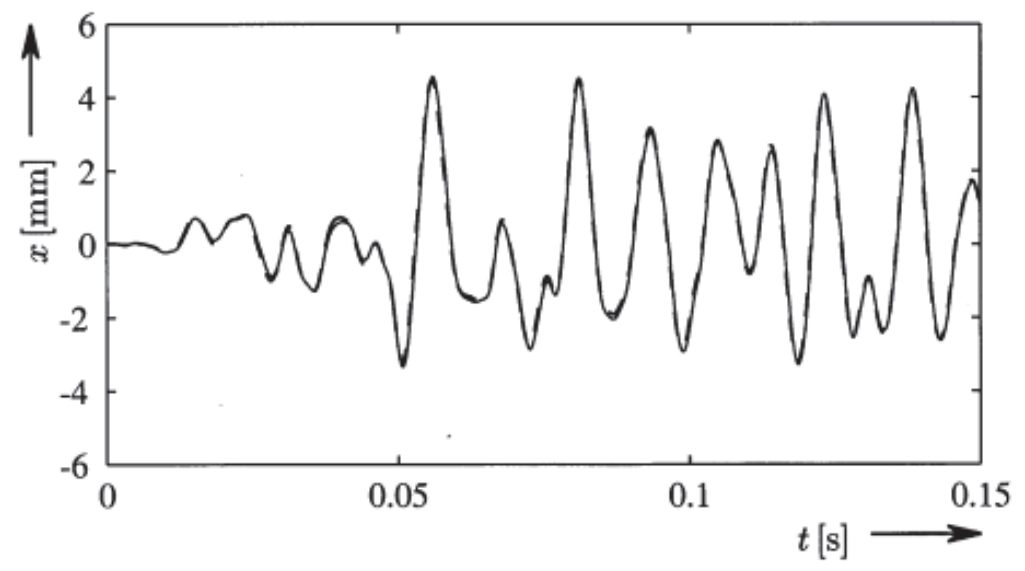

Figura 11: Sinais de saída do alto-falante real e modelo passivo. Extraído de [19].

é mencionado um ganho de $15 \mathrm{~dB}$ em relação ao caso linear, no qual as não linearidades do alto-falante não são devidamente excitadas pelo sinal de entrada.

\subsection{Modelo de Euler}

As idéias básicas por trás dos algoritmos que são usados para resolver numericamente conjuntos de equações diferenciais ordinárias especificadas por meio de um modelo de espaço de estado são apresentadas em [36]. Em particular, essa seção tem o objetivo principal de apresentar a discretização pelo método de Euler do modelo de parâmetros passivos descrito na seção anterior.

\subsubsection{Discretização pelo Método de Euler}

A representação do modelo de parâmetros passivos em equações de espaço de estados pode ser reescrita na forma

$$
\dot{x(t)}=f(x(t))+g(x(t)) u(t)
$$


Portanto, a discretização pelo método de Euler da equação (3.8) é dada por

$$
x(n+1)=x(n)+T f(x(n))+T g(x(n)) u(n)
$$

\subsubsection{Carga Computacional}

De acordo com a seção anterior, a representação do modelo físico do altofalante em equações de espaço de estados pode ser discretizada utilizando o método de Euler definido pela equação (3.9), como segue

$$
\left[\begin{array}{c}
x_{1}(n+1) \\
x_{2}(n+1) \\
x_{3}(n+1)
\end{array}\right]=\left[\begin{array}{c}
x_{1} \\
x_{2} \\
x_{3}
\end{array}\right]+T\left[\begin{array}{c}
x_{2} \\
\frac{1}{M}\left\{a\left(x_{1}\right) x_{3}+\frac{x_{3}^{2}}{2} \widetilde{L}^{\prime}\left(x_{1}\right)-k\left(x_{1}\right) x_{1}-G x_{2}\right\} \\
-\frac{1}{\widetilde{L}\left(x_{1}\right)}\left\{R x_{3}+\widetilde{L}^{\prime}\left(x_{1}\right) x_{2} x_{3}+a\left(x_{1}\right) x_{2}\right\}
\end{array}\right]+T\left[\begin{array}{c}
0 \\
0 \\
\frac{1}{\widetilde{L}\left(x_{1}\right)}
\end{array}\right] e
$$

onde por simplicidade a varíavel de tempo discreto $n$ de $x_{1}, x_{2}$ e $x_{3}$ foi omitida do lado direito da equação, e o vetor de estados é definido como

$$
\boldsymbol{x}=\left[\begin{array}{l}
x \\
\dot{x} \\
i
\end{array}\right]=\left[\begin{array}{l}
x_{1} \\
x_{2} \\
x_{3}
\end{array}\right]
$$

e as funções $a\left(x_{1}\right), k\left(x_{1}\right)$ e $\widetilde{L}\left(x_{1}\right)$ que modelam o comportamento do fator de força, da rigidez da suspensão do diafragma e da indutância não-linear da bobina de voz são dadas por:

$$
\begin{aligned}
& a\left(x_{1}\right)=\frac{a_{0}}{1+a_{1}\left(x_{1}-x_{a}\right)^{2}} \\
& k\left(x_{1}\right)=k_{0}+k_{1}\left(x_{1}-x_{k}\right)^{2}
\end{aligned}
$$




$$
\widetilde{L}\left(x_{1}\right)=b_{0}+b_{1} \tan ^{-1}\left(w_{l} x_{1}+\phi_{l}\right)
$$

A definição da carga computacional necessária para o cômputo da saída do modelo pode ser verificada pela contagem do número de operações relacionadas à implementação das equações descritas acima.

Além das operações básicas de multiplicação, divisão, adição e subtração identificadas por inspeção direta dessas equações, observa-se a presença da função $\tan ^{-1}(x)$ na equação (3.14). A determinação do número de operações relacionadas ao cômputo dessa função baseia-se na equação de aproximação utilizada no presente trabalho, definida em [37] e dada por

$$
\tan ^{-1}(x)=0,785 x+0,273 x(1-|x|)
$$

Em particular, a discretização de Euler é dependente da stiffness do sistema físico que foi modelado pelas equações de espaço de estado, pois essa propriedade influencia na determinação do intervalo de tempo $T$ mínimo necessário para implementar o sistema. Em geral, é necessária uma frequência de amostragem bem superior à frequência de amostragem original do sistema, o que resulta em um aumento significativo da carga computacional do modelo de Euler.

Portanto, o levantamento da carga computacional deve levar em conta as etapas de interpolação e decimação associadas à conversão de taxa de amostragem do modelo.

O modelo de Euler tem a carga computacional representada pelo número de multiplicações e divisões necessárias para o cômputo das equações de espaço de estado discretizadas. Os dados obtidos são apresentados na Tabela 5.

Destaca-se que a operação de divisão é mais complexa computacionalmente, 


\begin{tabular}{|c|c|}
\hline \multicolumn{2}{|c|}{ Carga Computacional } \\
\hline Equações & Operações \\
\hline $\mathrm{x}_{1}$ & 1 \\
$\mathrm{x}_{2}$ & 8 \\
$\mathrm{x}_{3}$ & 7 \\
$\mathrm{~A}\left(\mathrm{x}_{1}\right)$ & 4 \\
$\mathrm{k}\left(\mathrm{x}_{1}\right)$ & 2 \\
$\widetilde{L}\left(x_{1}\right)$ & 2 \\
$\arctan$ & 3 \\
$\widetilde{L}^{\prime}\left(x_{1}\right)$ & 6 \\
\hline \multicolumn{2}{|c|}{ Nopers $=33$} \\
\hline
\end{tabular}

Tabela 5: Número de operações do modelo de Euler.

sendo mais díficil de ser implementada que a multiplicação. Assim, para efeitos de simplificação considerou-se a divisão como 2 operações e a multiplicação como apenas 1.

\subsection{Sistema Bilinear}

Os sistemas bilineares são uma classe especial de sistemas não-lineares que são lineares na entrada e lineares no estado, mas não conjuntamente lineares no estado e na entrada. Especificamente, um sistema bilinear invariante no tempo de entrada única e saída única (SISO), pode ser representado como segue [38]:

$$
\begin{gathered}
\dot{\mathbf{x}}(t)=\mathbf{F} \mathbf{x}(t)+\mathbf{G} \mathbf{x}(t) u(t)+\mathbf{b} u(t) \\
y(t)=\mathbf{c}^{T}(t) \mathbf{x}(t)
\end{gathered}
$$

onde $t$ é a variável tempo, $N$ é a dimensão do vetor de espaço de estados $\mathbf{x}(t)$, $\mathbf{x}(0)=0$ é a condição inicial, $\mathbf{F}$ e $\mathbf{G}$ são matrizes $N \mathbf{x} N, \mathbf{c}(t), \mathbf{x}(t)$ e b são vetores colunas N-dimensionais.

Sistemas bilineares surgem como modelos naturais para uma variedade de processos físicos e biomédicos, devido ao fato de poderem ser tratados analiti- 
camente e serem capazes de aproximar qualquer relação de entrada/saída em intervalos de tempo finitos. Por isso, são largamente empregados na resolução de problemas práticos [18].

Retomando a equação (3.8), a representação do modelo de parâmetro passivos em equações de espaço de estados do alto-falante pode ser reescrita na forma

$$
\mathbf{x}(t)=\mathbf{f}(\mathbf{x}(t))+\mathbf{g}(\mathbf{x}(t)) u(t)
$$

Através do Teorema de Carleman, um sistema não-linear da forma descrita por (3.18) pode ser aproximado por um sistema bilinear da forma (3.16). A bilinearização de Carleman permite que a não-linearidade seja explicitada e sistematicamente incorporada na aproximação do sistema bilinear [38].

\subsubsection{Discretização do Sistema Bilinear}

Para implementação do sistema bilinear utiliza-se o toolbox computacional a fim de se obter as matrizes F e G necessárias para a resolução da equação (3.16). Entretanto, a versão discretizada da equação (3.16) deve ser considerada para uma implementação prática do sistema bilinear.

Aplicando o método de discretização de Euler sobre a equação (3.16) como mencionado em [39] (mais precisamente na seção 1.8), tem-se

$$
\mathbf{x}(n+1)=\mathbf{x}(n)+T\{(\mathbf{F}+\mathbf{G} u(n)) \mathbf{x}(n)+\mathbf{b} u(n)\}
$$

É notável que as matrizes $\mathbf{F}$ e $\mathbf{G}$ associadas ao modelo físico real do altofalante apresentam um alto grau de esparsidade. Tal fato motiva o uso de um algoritmo para implementação do sistema bilinear utilizando-se dessa propriedade para, dessa maneira, reduzir a sua complexidade computacional substanci- 
almente. O algoritmo é descrito a seguir e o respectivo script do Matlab, que o implementa, é apresentado no apêndice G.

Considerando que os vetores $\mathbf{F}_{s}$ e $\mathbf{G}_{s}$ contêm apenas os elementos não nulos das matrizes $\mathbf{F}$ e $\mathbf{G}$, e foram obtidos através da função find do Matlab, após a aplicação do algoritmo sob o sistema bilinear da equação (3.19) resulta em

$$
\mathbf{x}(n+1)=\mathbf{x}(n)+T\left\{\left(\mathbf{F}_{r}+\mathbf{G}_{r} u(n)\right)+\mathbf{b} u(n)\right\}
$$

onde $\mathbf{F}_{r}$ e $\mathbf{G}_{r}$ são vetores obtidos através do produto entre os elementos dos vetores, $\mathbf{F}_{s}$ e $\mathbf{G}_{s}$, e seus elementos correspondentes do vetor $\mathbf{x}(n)$.

\subsubsection{Carga Computacional}

As observações sobre carga computacional feitas na seção anterior para discretização pelo método de Euler continuam valendo para o sistema bilinear.

Logo, a definição da carga computacional necessária para o cômputo da saída do modelo pode ser verificada pela cálculo do número de operações relacionadas à implementação da equação (3.19).

Além disso, a influência dos processos de interpolação e decimação deve ser levada em conta, já que a propriedade de stiffness do sistema físico determina que esses processos estejam presentes na implementação do modelo Bilinear.

Utilizando o algoritmo descrito no apêndice G sobre o sistema bilinear discretizado pelo método de Euler, conforme explicado na seção 3.3.1, resulta no número de operações por amostra do sinal de entrada apresentado na Tabela 6, a qual revela as dimensões e número de elementos de matrizes e vetores envolvidos nesse cálculo. 


\begin{tabular}{|c|c|c|}
\hline \multicolumn{3}{|c|}{ Carga Computacional } \\
\hline Variável & Dimensões & Nelem \\
\hline $\mathbf{F}$ & $34 \times 34$ & 1156 \\
$\mathbf{G}$ & $34 \times 34$ & 1156 \\
$\mathbf{F}_{(s)}$ & $142 \times 1$ & 142 \\
$\mathbf{G}_{(s)}$ & $14 \times 1$ & 14 \\
$\mathbf{F}_{(r)}$ & $34 \times 1$ & 34 \\
$\mathbf{G}_{(r)}$ & $34 \times 1$ & 34 \\
$\mathbf{b}$ & $34 \times 1$ & 34 \\
$\mathbf{x}(n)$ & $34 \times 1$ & 34 \\
\hline \multicolumn{3}{|c|}{ Nopers $=258$} \\
\hline
\end{tabular}

Tabela 6: Número de operações do Sistema Bilinear

\subsection{Filtro de Volterra Convencional}

Como mencionado em [40], a expansão da série de Volterra pode ser interpretada como uma expansão da série de Taylor com memória, na qual o sinal de saída está relacionado ao sinal de entrada de forma não-recursiva da seguinte forma:

$$
\begin{aligned}
y(n)= & w_{0}+\sum_{l_{1}=0}^{\infty} w_{1}\left(l_{1}\right) x\left(n-l_{1}\right) \\
& +\sum_{l_{1}=0}^{\infty} \sum_{l_{2}=0}^{\infty} w_{2}\left(l_{1}, l_{2}\right) x\left(n-l_{1}\right) x\left(n-l_{2}\right) \\
& +\sum_{l_{1}=0}^{\infty} \sum_{l_{2}=0}^{\infty} \sum_{l_{3}=0}^{\infty} w_{3}\left(l_{1}, l_{2}, l_{3}\right) x\left(n-l_{1}\right) x\left(n-l_{2}\right) x\left(n-l_{3}\right)+\ldots \\
& +\sum_{l_{1}=0}^{\infty} \sum_{l_{2}=0}^{\infty} \ldots \sum_{l_{p}=0}^{\infty} w_{p}\left(l_{1}, l_{2}, \ldots, l_{p}\right) x\left(n-l_{1}\right) x\left(n-l_{2}\right) \ldots x\left(n-l_{p}\right)+\ldots
\end{aligned}
$$

onde $w_{i}\left(l_{1}, l_{2}, \ldots, l_{i}\right)$, para $i=0,1, \ldots$, são os coeficientes do modelo não-linear baseado na série de Volterra, também chamados de núcleos de Volterra e $y(n)$ representa a saída do sistema desconhecido.

Para simular computacionalmente sistemas através de séries de Volterra, é ne- 
cessário implementar o modelo de Volterra do sistema e isto é realizado usando um algoritmo de processamento de sinal denominado Filtro de Volterra. No entanto, o Filtro de Volterra requer a implementação de convoluções multidimensionais de carga computacional elevada, fato que inviabiliza sua implementação na forma original, sem truncamento, para aplicações que exigem desempenho em tempo real.

Ao truncar a série, pode-se reduzir a complexidade computacional, sacrificando a precisão da expansão em série. Com uma ordem reduzida, a representação da série de Volterra é ainda complexa, mesmo quando as ordens da série e o filtro são moderados. Contudo, a implementação do Filtro de Volterra de $2^{\mathrm{a}}$ e $3^{\mathrm{a}}$ ordens pode trazer bons resultados em alguns casos.

Os filtros de Volterra são amplamente utilizados pelo NLAEC por causa da sua estrutura genérica, que pode ser considerada como uma simples generalização de filtros adaptativos lineares [40]. Em geral, algoritmos e métodos adaptativos são utilizados para a estimação de um núcleo de Volterra, sendo que a precisão das estimativas dos núcleos de Volterra determina a precisão do modelo do sistema [29].

Por outro lado, como alternativa ao uso do processo adaptivo para obtenção dos núcleos de Volterra, pode-se empregar o método de bilinearização de Carleman a fim de que a dinâmica não-linear do sistema possa ser representada por um conjunto de equações de espaço de estados na forma bilinear e, assim, proceder com o cálculo dos núcleos de Volterra correspondentes de forma direta como descrito em [14].

No presente trabalho, utilizam-se modelos de Volterra derivados do modelo paramétrico passivo do alto-falante, descrito na seção 3.1, representado por equações de espaço de estados na forma bilinear, sendo que os respectivos núcleos de Volterra são calculados com o uso do método de bilinearização de Carleman. 
A demostração das expressões para cálculo dos núcleos, assim como as demais particularidades com relação aos modelos, podem ser verificadas em [18].

\subsubsection{Carga Computacional}

Os filtros de Volterra são capazes de modelar uma grande variedade de sistemas físicos, porém o número de coeficientes necessários para representar esses sistemas cresce muito rapidamente tanto com o aumento das extensões de memória de seus núcleos quanto com sua ordem ou grau de não-linearidade. Tal fato pode ser verificado pela análise a seguir.

Um filtro de Volterra causal e invariante no tempo consiste em um modelo matemático não-linear de tempo discreto cuja relação entrada/saída é definida como

$$
y(n)=\sum_{p=1}^{P} \sum_{k_{1}=0}^{N_{p}-1} \cdots \sum_{k_{p}=0}^{N_{p}-1} h_{p}\left(k_{1}, \ldots, k_{p}\right) u\left(n-k_{1}\right) \ldots u\left(n-k_{p}\right)
$$

onde as funções $h_{p}:\left\{0,1, \ldots, N_{p}-1\right\}^{p} \rightarrow \mathbb{R}$ são os núcleos do Filtro de Volterra, $N_{p}$ é a extensão de memória do núcleo de ordem $p$, sendo que $u$ é a entrada e $y$ é a saída.

Por inspeção da equação acima pode-se notar que a carga computacional associada ao cálculo do componente de ordem p da saída é $\mathrm{O}\left(N_{p}^{p}\right)$ por amostra, isto é, a carga computacional cresce exponencialmente com a ordem do filtro.

Admitindo-se uma forma triangular para os núcleos, isto é, $h_{p}\left(k_{1}, \ldots, k_{p}\right)=0$ se $k_{i}>k_{j}$ para algum $i<j$, obtem-se o número mínimo de operações aritméticas necessárias para o cômputo da saída, considerando a ordem e as extensões de memórias dos núcleos do Filtro de Volterra como fixas. Verifica-se que o pressuposto de triangularidade modifica os limites inferiores dos somatórios da equação 
(3.22), a qual pode ser redefinida como:

$$
y(n)=\sum_{p=1}^{P} \sum_{k_{1}=0}^{N_{p}-1} \ldots \sum_{k_{p}=k_{p-1}}^{N_{p}-1} h_{p}\left(k_{1}, \ldots, k_{p}\right) u\left(n-k_{1}\right) \ldots u\left(n-k_{p}\right)
$$

Devido à redundância existente na definição do núcleo de ordem $p$ para $p>1$, o número de coeficientes necessários para caracterizar o filtro de Volterra com núcleos triangulares é menor do que $N_{p}^{p}$, o que pode ser verificado pela expressão $(3.24)$.

$$
\Psi_{F V}=\sum_{p=1}^{P}\left(\begin{array}{c}
N_{p}+p-1 \\
p
\end{array}\right)=\sum_{p=1}^{P} \frac{\left(N_{p}+p-1\right) !}{p !\left(N_{p}-1\right) !}
$$

onde $P$ é a ordem do filtro $\mathrm{FV}, N_{p}$ é a extensão de memória do núcleo de ordem $p$, sendo que $p \in 1, \ldots, P$.

Essa expressão fornece o número das diferentes combinações dos índices $k_{i}$ necessárias para descrever os núcleos triangulares. Nota-se que se trata da fórmula de combinação com reposição, já que apenas uma permutação de determinados índices $k_{i}, \ldots, k_{p}$ conta para o resultado e pode haver $p-1$ repetições de valores nos índices $k_{i}, \ldots, k_{p}$.

Considerando apenas as operações de multiplicações relativas a cada termo no somatório na equação (3.23), que define a componente $p$ da saída do Filtro de Volterra com núcleos na forma triangular, é possível obter a expressão de carga computacional dada pela equação (3.25).

$$
\text { Nopers }=\sum_{p=1}^{P}\left(\frac{\left(N_{p}+p-1\right) !}{p !\left(N_{p}-1\right) !}\right) p
$$

onde $P$ é a ordem do filtro de Volterra, $N_{p}$ é a extensão de memória do núcleo de ordem $p$, sendo que $p \in 1, \ldots, P$. 


\subsection{Filtro de Volterra Esparso Interpolado}

O esquema esparso com interpolação proposto em [20] é uma maneira de diminuir a complexidade do filtro de Volterra convencional admitindo-se um certo nível de redundância nos seus núcleos, mais especificamente, entre coeficientes vizinhos.

Assim, o esquema propõe a redução dos graus de liberdade de cada um dos núcleos do filtro de Volterra convencional, ou seja, promove a compactação do modelo através do descarte de parte dos coeficientes de cada um dos núcleos. Por último, um filtro interpolador é acrescentado ao modelo antes do filtro de Volterra para que os termos da saída do filtro correspondentes aos coeficientes descartados sejam estimados.

Afim de analisar o esquema esparso interpolado de forma objetiva e intuitiva, apresenta-se inicialmente o Filtro FIR Esparso Interpolado (SIFIR), o qual serve de base para o entendimento desse esquema aplicado ao Filtro de Volterra [20]. O diagrama em blocos do SIFIR é apresentado na Figura 12.

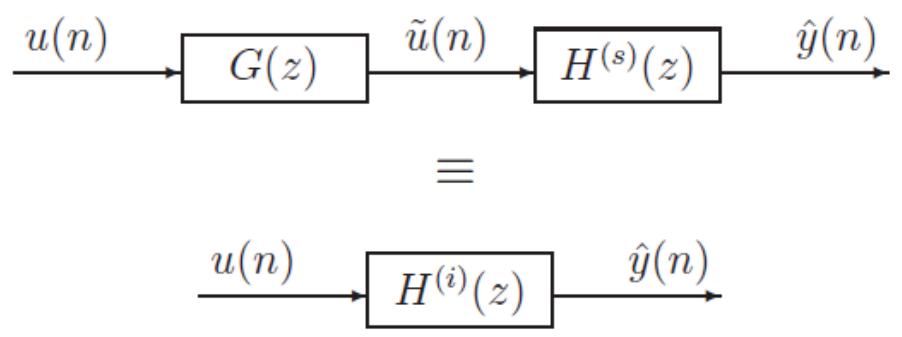

Figura 12: Esquema esparso com interpolação para aproximação de um filtro FIR. Extraído de [20].

Seja $h(n)$ um filtro FIR de comprimento $N$ pode-se implementar o esquema esparso com interpolação através da substituição dos coeficientes $h(n)$ do filtro 
por uma versão esparsa $h^{s}(n)$ com fator de interpolação $L \in \mathbb{N}^{*}$, definida como

$$
h^{s}(n)=\left\{\begin{array}{cc}
h(n), & \text { se } \exists k \in \mathbb{N} \text { tal que } \mathrm{n}=k L, \\
0, & \text { caso contrário }
\end{array}\right.
$$

Em seguida, um filtro interpolador com resposta impulsiva $g(n)$ de comprimento $M$ é introduzido, sendo que sua saída é dada por

$$
\tilde{u}(n)=\sum_{k=0}^{M-1} g(k) u(n-k)
$$

Assim, a saída do filtro FIR esparso interpolado pode ser representado por

$$
\check{y}(n)=\sum_{k=0}^{N-1} g(k) \tilde{u}(n-k)
$$

sendo que a saída original do filtro FIR é dada por

$$
y(n)=\sum_{k=0}^{N-1} g(k) u(n-k)
$$

O diagrama de blocos da Fig. 12 representa o filtro esparso interpolado cujos coeficientes podem ser obtidos através da expressão

$$
h^{(i)}=\sum_{k=0}^{M-1} g(k) h^{(s)}(n-k)
$$

O diagrama de blocos do Filtro de Volterra Esparso Interpolado é representado na Figura 13 e as equações generalistas para obtenção desse filtro de ordem P são apresentadas a seguir. De forma geral, o esquema de obtenção do Filtro de Volterra Esparso Interpolado pode ser verificado em maior detalhe em [20].

A forma compacta do Filtro de Volterra Esparso Interpolado é mostrada na parte de cima da Figura 13. Os sinais de entrada e saída são $x(n)$ e $\hat{y}(n)$, 


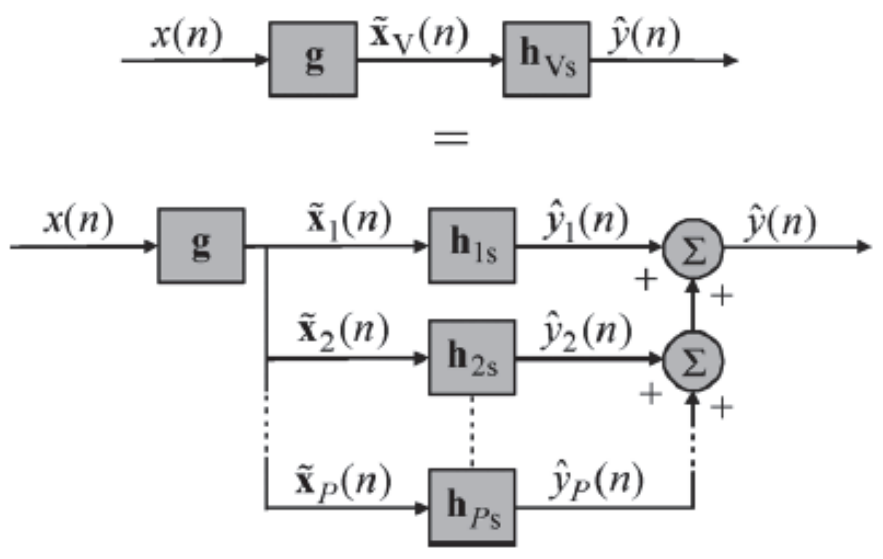

Figura 13: Diagrama em blocos do Filtro de Volterra Esparso Interpolado. Extraído de [20].

respectivamente; g é o vetor de interpolação; o vetor de entrada de Volterra interpolado $\tilde{\mathbf{x}}_{V}(n)$ e o vetor de coeficientes de Volterra esparso são dados pelas equações a seguir

$$
\begin{gathered}
\tilde{\mathbf{x}}_{V}(n)=\left[\tilde{\mathbf{x}}_{1}^{T}(n) \tilde{\mathbf{x}}_{2}^{T}(n) \ldots \tilde{\mathbf{x}}_{P}^{T}(n)\right]^{T} \\
\mathbf{h}_{V s}(n)=\left[\mathbf{h}_{1 s}^{T}(n) \mathbf{h}_{2 s}^{T}(n) \ldots \mathbf{h}_{P s}^{T}(n)\right]^{T}
\end{gathered}
$$

resultando na seguinte relação de entrada/saída

$$
\hat{y}(n)=\mathbf{h}_{V s}(n) \tilde{\mathbf{x}}_{V}(n)
$$

A parte de baixo da Figura 13 representa a forma expandida do Filtro de Volterra Esparso Interpolado, onde $\tilde{\mathbf{x}}_{p}(n)$ representa o $p$-ésimo vetor de entrada interpolado, $\mathbf{h}_{p s}(n)$ é o $p$-ésimo bloco de coeficientes esparso e $\hat{y}_{p}(n)$ é o sinal de saída de cada $p$-ésimo bloco esparso interpolado.

Um Filtro de Volterra Esparso de primeira ordem $(\mathrm{P}=1)$ é equivalente ao ao filtro SIFIR. Então, o sinal de saída para o bloco de primeira ordem esparso 
interpolado é

$$
\hat{y}_{1}(n)=\tilde{\mathbf{x}}_{1}(n)^{T} \mathbf{h}_{1 s}
$$

e $\mathbf{h}_{1 s}$ e $\tilde{\mathbf{x}}_{1}(n)$ são dados por

$$
\begin{gathered}
\mathbf{h}_{1 s}(n)=\left[h_{1}(0) 0 \ldots h_{1}(L) 0 \ldots h_{1}(L) 0 \ldots \ldots h_{1}\left[\left(N_{s}-1\right) L\right] 0 \ldots 0\right]^{T} \\
\tilde{\mathbf{x}}_{1}(n)=[\tilde{x}(n) \tilde{x}(n-1) \ldots \tilde{x}(n-N+1)]^{T}
\end{gathered}
$$

O vetor de interpolação é dado por $\mathbf{g}=[g(0) g(1) \ldots g(M-1)]^{T}$ e a matriz de interpolação $\mathrm{G}$ por

$$
\mathbf{G}=\left[\begin{array}{ccccc}
g(0) & 0 & 0 & \ldots & 0 \\
g(1) & g(0) & 0 & \ldots & 0 \\
g(2) & g(1) & g(0) & \ldots & 0 \\
\vdots & \vdots & \vdots & \ddots & \vdots \\
g(M-1) & g(M-2) & g(M-3) & \ldots & g(0) \\
0 & g(M-1) & g(M-2) & \ldots & g(1) \\
0 & 0 & g(M-1) & \ldots & g(2) \\
\vdots & \vdots & \vdots & \ddots & \vdots \\
0 & 0 & 0 & 0 & g(M-1)
\end{array}\right]
$$

Considerando o vetor de entrada estendido

$$
\mathbf{x}_{e}(n)=[x(n) x(n-1) \ldots x(n-N-M+2)]^{T}
$$


a equação (3.36) pode ser expressa por

$$
\tilde{\mathbf{x}}_{1}(n)=\mathbf{G}^{T} \mathbf{x}_{e}(n)
$$

\subsubsection{Carga Computacional}

O número total de coeficientes envolvidos na representação esparsa com interpolação é igual a um Filtro de Volterra com núcleos que possuem extensões de memórias dadas por

$$
N_{p}^{(s)}=\left(\frac{N_{p}-1}{L}\right)+1
$$

onde $L$ é o fator de interpolação utilizado e $N_{p}$ é a extensão de memória do núcleo de ordem $p$ original.

Considerando apenas as operações de multiplicações relativas à cada termo no somatório na equação (3.23), analogamente ao Filtro de Volterra Convencional, obtemos a expressão de carga computacional dada pela equação (3.41).

$$
\text { Nopers }=\sum_{p=1}^{P}\left(\frac{\left(N_{p}^{(s)}+p-1\right) !}{p !\left(N_{p}^{(s)}-1\right) !}\right) p+(2 L-1)
$$

onde $P$ é a ordem do filtro de Volterra Esparso Interpolado, $N_{p}^{(s)}$ é a extensão de memória do núcleo de ordem $p$, sendo que $p \in 1, \ldots, P$.

Portanto, existe a necessidade de se analisar a contribuição de algumas operações adicionais $(2 L-1)$ para o cômputo da saída do filtro interpolador a fim de se determinar a carga computacional total.

Embora o filtro interpolador normalmente possua uma resposta impulsiva de curta duração, nota-se que essas operações adicionais tornam-se significativas à medida que a ordem do filtro permanece baixa, em geral, nos casos em que 
$p<=3$. Nos demais casos, essa contribuição para a carga computacional total do Filtro de Volterra Esparso Interpolado é insignificante. 


\subsection{Modelo PARAFAC-Volterra}

O modelo de Wiener paralelo em cascata com $N$ ramificações permite aproximar com certa precisão qualquer sistema não-linear de tempo discreto de memória finita [41] e pode ser descrito pela seguinte relação de entrada-saída:

$$
y(k)=\sum_{n=1}^{N} \eta^{(n)}\left(\sum_{m=1}^{M} h_{m}^{(n)} u_{k-m}\right)
$$

onde $h^{(n)}$ e $\eta^{(n)}($.$) são, respectivamente, o bloco linear FIR e a não-linearidade$ estática da n-ésima ramificação, $n=1, \ldots, N$. Cada filtro linear FIR $h_{m}$ associado à $y(k)$ possui extensão de memória $M$.

O modelo PARAFAC-Volterra é um modelo de Wiener paralelo em cascata para o qual os filtros lineares FIR $h_{m}$ são dados pelas colunas dos fatores matriciais das assim chamadas decomposições PARAFAC dos núcleos, e as não-linearidades estáticas $\eta^{(n)}($.$) são potências simples (.)^{n}[21]$.

De acordo com [21] qualquer kernel de Volterra de ordem $p$, com $p \geqslant 2$, pode ser decomposto usando o modelo PARAFAC simétrico, com rank $r_{p}$ e fator matricial $A^{(p)} \in \mathbb{C}^{M_{p} x r_{p}}$ simétricos:

$$
h_{m_{1}, \cdots, m_{p}}^{(p)}=\sum_{r=1}^{r_{p}} \prod_{i=1}^{p} a_{m_{i}, r}^{(p)} .
$$

Logo, um filtro de Volterra com núcleo simétrico baseado na decomposição PARAFAC tem sua saída de ordem $p$ descrita por [21]:

$$
\begin{aligned}
y_{k}^{(p)} & =\sum_{m_{1}=1}^{M_{p}} \cdots \sum_{m_{1}=1}^{M_{p}} h_{m_{1}, \cdots, m_{p}}^{(p)} \prod_{i=1}^{p} u_{k-m_{i}} \\
& =\sum_{m_{1}=1}^{M_{p}} \cdots \sum_{m_{1}=1}^{M_{p}}\left(\sum_{r=1}^{r_{p}} \prod_{i=1}^{p} a_{m_{i}, r}^{p}\right) \prod_{i=1}^{p} u_{k-m_{i}} .
\end{aligned}
$$


Rearranjando a ordem dos somatórios tem-se

$$
y_{k}^{(p)}=\sum_{r=1}^{r_{p}} \prod_{i=1}^{p} \sum_{m_{1}=1}^{M_{p}} a_{m_{i}, r}^{(p)} u_{k-m_{i}} .
$$

Definindo-se o vetor de entrada como

$$
u_{k}^{(p)}=\left[u_{k-1}, \cdots, u_{k-M_{p}}\right]^{T},
$$

a expressão de $y_{k}^{(p)}$ se torna

$$
y_{k}^{(p)}=\sum_{r=1}^{r_{p}}\left(u_{k}^{(p) T} A_{r}^{(p)}\right)^{T} .
$$

Assim, o termo homogêneo de ordem $p$ pode ser decomposto em $r_{p}$ modelos de Wiener em paralelo, cada qual sendo associado a uma coluna $A_{r}^{(p)} \in \mathbb{C}^{M_{p} x 1}$ do fator matricial $A^{(p)}$ da decomposição de PARAFAC do kernel de ordem $p$.

Com isso, a representação em diagrama de blocos do modelo PARAFACVolterra pode ser apresentada da forma

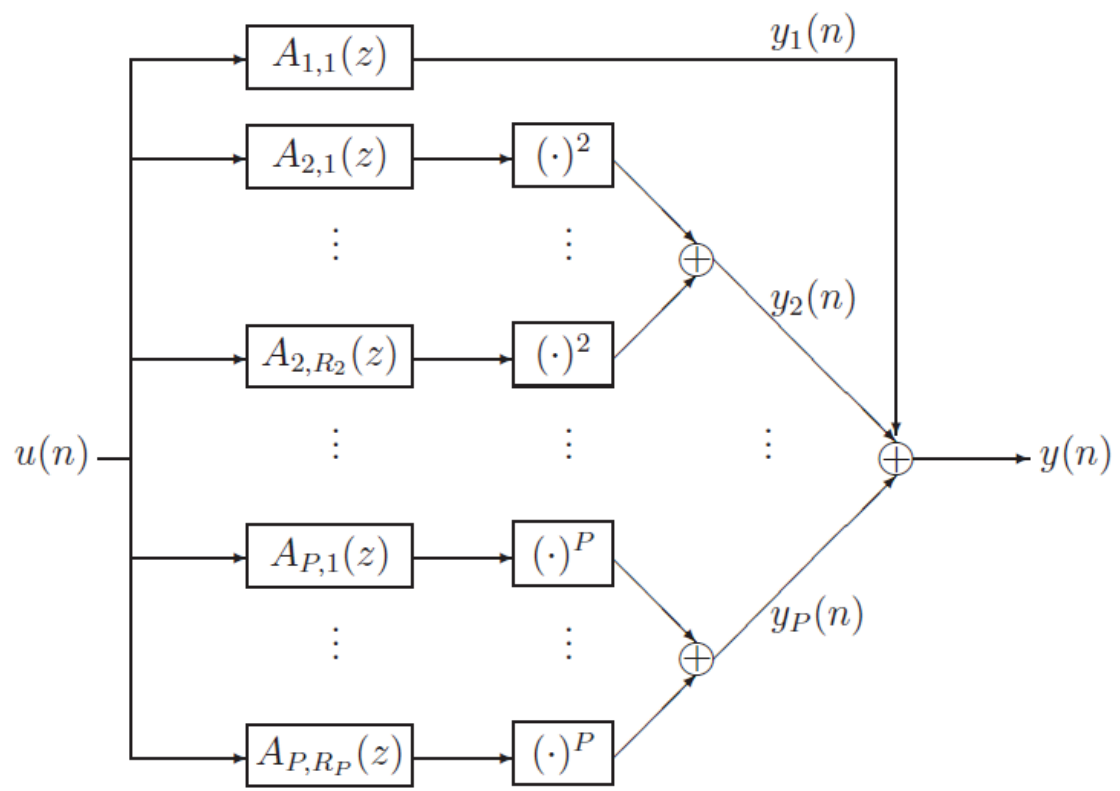

Figura 14: Modelo PARAFAC-Volterra. Extraído de [18]. 


\subsubsection{Carga Computacional}

A redução de complexidade estrutural obtida com a adoção da estrutura PARAFAC-Volterra de ordem $P$ depende dos postos $R_{2}, \ldots, R_{p}$ suficientes para se obter uma aproximação satisfatória dos núcleos do Filtro de Volterra Convencional. Considerando para fins de comparação um Filtro de Volterra Convencional de ordem $P$, cujos núcleos possuem extensões de memória $N_{p} \operatorname{com} p \in\{1, \ldots, P\}$, o número de coeficientes necessários para se caracterizar um modelo PARAFACVolterra completamente é

$$
\Psi_{P V M}=N_{1}+\sum_{p=2}^{P} R_{p} N_{p}
$$

Para se computar a componente de ordem $p$ da saída $y_{p}$, com $p>=2$, são requeridas $R_{p}$ filtragens lineares $\left(R_{p} N_{p}\right),(p-1) R_{p}$ multiplicações relativas às potências e $R_{p}-1$ adições para agregar as saídas dos filtros. Assim, o número de operações por amostra é da ordem de $\mathrm{O}\left(R_{p}\left(N_{p}+p\right)\right)$, considerando a im-

plentação direta dos filtros lineares. Para $p=1$ é requerida apenas 1 filtragem linear, resultando na ordem $\mathrm{O}\left(N_{1}\right)$. Logo, a carga computacional total do modelo PARAFAC-Volterra é da ordem de $\mathrm{O}\left(N_{1}+R_{p}\left(N_{p}+p\right)\right)$.

Considerando apenas as operações de multiplicação para se computar a componente de ordem $p$ da saída $y_{p}$ do modelo PARAFAC-Volterra resulta na expressão de carga computacional dada pela equação (3.49).

$$
\text { Nopers }=N_{1}+\sum_{p=2}^{P} R_{p}\left(N_{p}+p-1\right)
$$




\section{EFEITO DE NÃO-LINEARIDADES EM ALGORITMOS DE CANCELAMENTO DE ECO ACÜSTICO LINEAR}

Nesse capítulo será verificado como não-linearidades, sempre presentes em maior ou menor grau, afetam o desempenho de algoritmos de cancelamento de eco acústico linear.

\subsection{Condicionamento de Sinal e Velocidade de Convergência}

A função de correlação é uma medida de como estatisticamente duas funções são semelhantes. A função de autocorrelação de um sinal aleatório é definida como o valor esperado desse sinal no tempo $n$ multiplicado pelo valor do seu conjugado complexo num momento diferente $m$. Isto é mostrado na equação (4.1), para instantes de tempo arbitrários, $n$ e $m$.

$$
\phi_{x x}(m)=E\left[x(n) x^{*}(n-m)\right]
$$

O condicionamento de uma matriz é definido como a razão entre o seu maior e seu menor valores singulares. No caso de uma matriz simétrica positiva semidefinida, como uma matriz de autocorrelação, os seus valores singulares são iguais aos seus autovalores. 


\subsection{Indicadores de Desempenho}

Os aspectos de interesse mais prioritários ao se analisar o desempenho de dispositivos canceladores de eco são:

- Tempo de convergência: é o tempo necessário para o AEC atingir um nível aceitável de eco residual constante.

- Grau de cancelamento: é a medida da quantidade de eco cancelado pelo AEC, medido geralmente em ERLE (Echo Return Loss Enhancement).

\subsubsection{Tempo de Convergência}

Para obter um desempenho satisfatório, o cancelador de eco deve ter uma taxa de convergência rápida, ou seja, um tempo de convergência pequeno, de modo que possa acompanhar adequadamente as modificações abruptas no sistema e as características do sinal.

O algoritmo LMS é o mais utilizado dentre os algoritmos de gradiente estocásticos e um fator que influencia diretamente sua velocidade de convergência é a característica de espalhamento dos autovalores da matriz de correlação do sinal de entrada [31]. De modo geral, quando este espalhamento é elevado o algoritmo converge lentamente.

\subsubsection{Grau de Cancelamento}

A intensidade do eco é medida em termos da perda do retorno de eco (ERL) definida como uma relação do sinal transmitido e do eco retornado medido em dB [ITU-G165]. É importante salientar que quanto maior for a perda de retorno 
de eco, menor será a potência do eco.

$$
\boldsymbol{E R L}=10 \log _{10}\left(\frac{\text { Potência do sinal transmitido }}{\text { Potência do eco retornado }}\right)
$$

A métrica mais reconhecida para avaliar o desempenho do AEC, e que representa o grau de cancelamento de eco, é o parâmetro que mede a melhoria na perda do retorno de eco (ERLE) em regime permanente e é definido como:

$$
\boldsymbol{E} \boldsymbol{R} \boldsymbol{L} \boldsymbol{E}(d B)=10 \log _{10}\left(\frac{\sigma_{d}^{2}}{\sigma_{e}^{2}}\right)
$$

onde $\sigma_{d}^{2}$ e $\sigma_{e}^{2}$ referem-se às variâncias dos sinais desejado e de erro, $d(n)$ e $e(n)$, respectivamente.

A equação (4.3) é válida quando $d(n)$ não contém interferências; e resulta das definições acima que a melhoria da perda de retorno de eco (ERLE) é a diferença do ERL antes e após a aplicação de cancelamento de eco [22].

\subsection{Cancelador de Eco Acústico Linear}

Cancelador de Eco Acústico Linear (LAEC) é o dispositivo de cancelamento de eco acústico que usa algum tipo de filtro linear adaptativo para eliminar o eco em um sistema de comunicação. Genericamente, o LAEC pode ser representado pela Figura 15.

O sistema $\mathbf{W}_{o}$ representa a convolução das seguintes respostas impulsivas: do filtro de reconstrução do conversor $\mathrm{D} / \mathrm{A}$, do ambiente acústico, do microfone e do filtro anti-rebatimento do conversor A/D. Em particular, a resposta impulsiva do ambiente consiste do sinal medido no microfone quando um sinal impulsivo é emitido pelo alto-falante, sofrendo após isso diversas reflexões no ambiente da sala. Para maior simplicidade, consideramos que $\mathbf{W}_{o}$ é dado apenas pela 


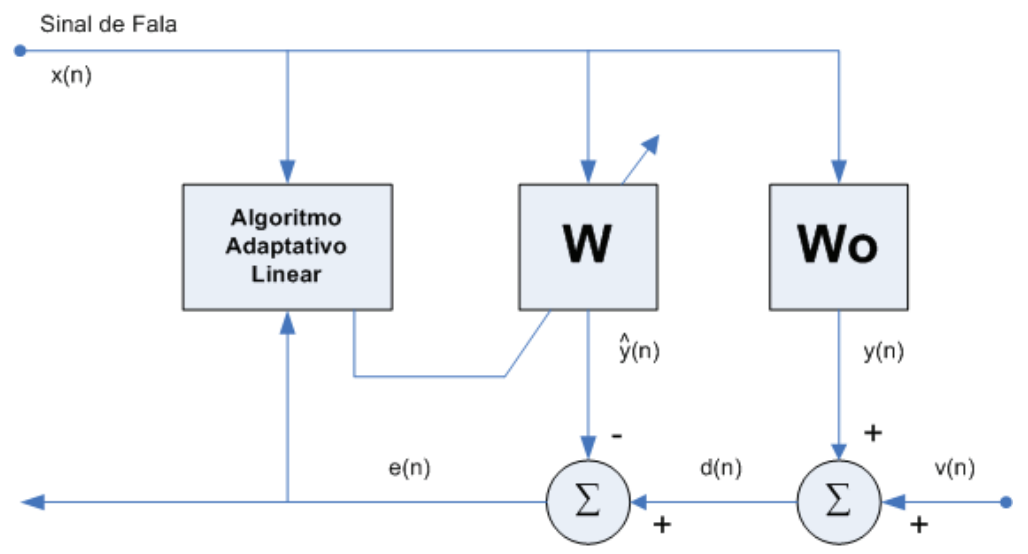

Figura 15: LAEC com alto-falante ideal.

resposta impulsiva do ambiente, isto é, que os demais fatores que determinam $\mathbf{W}_{o}$ são ideais. O alto-falante é considerado em separado de $\mathbf{W}_{o}$ e, inicialmente, assume-se que também é ideal.

Nota-se pela Figura 15 que o problema do cancelamento de eco acústico resume-se na identificação do sistema $\mathbf{W}_{o}$ através de um método de computação recursiva que se utiliza de um algoritmo adaptativo específico.

A cada iteração o algoritmo adaptativo recursivo gera um sistema $\mathbf{W}$, que é uma estimativa do sistema real $\mathbf{W}_{o}$, usando para isso: o sinal de fala $x(n)$, o sinal desejado $d(n)$, o erro residual $e(n)$ e a estimativa passada dos coeficientes $\mathbf{w}$.

Referencia-se, daqui por diante, esse caso como: AEC com auto-falante ideal - AEC(IL, do inglês Ideal Loudspeaker).

Este trabalho trata do efeito das características, possivelmente não-lineares, do alto-falante no cancelamento de eco acústico. Para isso, consideramos o diagrama do sistema dado pela Figura 16.

Observa-se que o alto-falante é representado explicitamente na Figura 16, fazendo com que o sinal de entrada do filtro FIR $\mathbf{W}_{o}$ passe a ser um sinal $\mathbf{x}^{\prime}(n)$, fornecido pela saída do alto-falante.

Três modelos de alto-falante são considerados: 


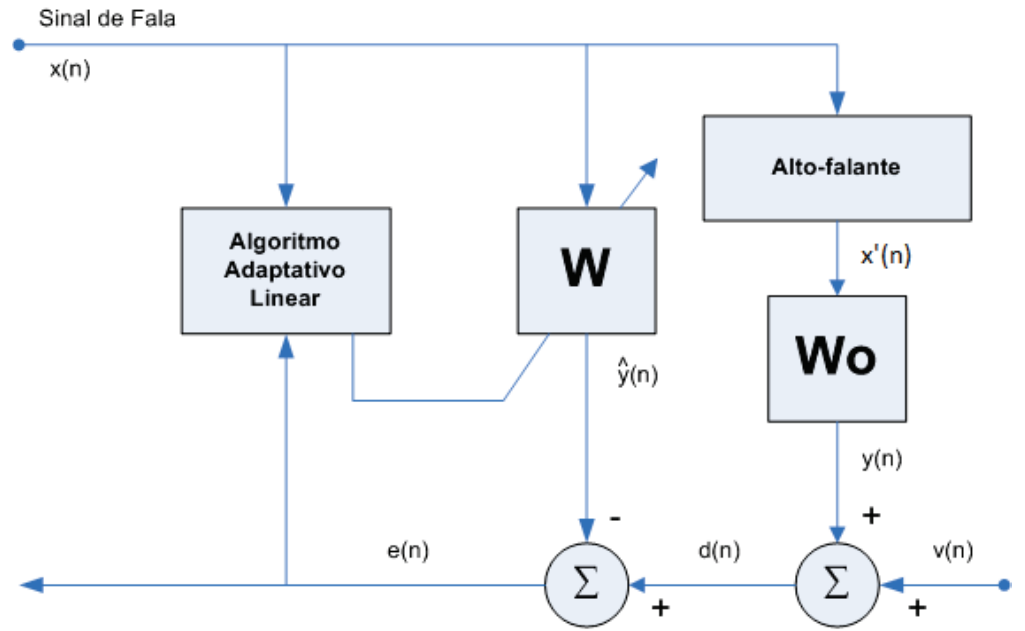

Figura 16: LAEC usando um modelo do alto-falante.

1. Alto-falante não-linear sem memória (NLIL, do inglês Nonlinear Instantaneous Loudspeaker).

O modelo em questão vem sendo largamente empregado na análise de cancelamento de eco não-linear $[4,42,2]$ e pode ser representado pela expressão:

$$
x_{n l}=x(n)+\alpha x^{2}(n)+\beta x^{3}(n)
$$

onde $x_{n l}$ é a saída não-linear do alto-falante; $\alpha$ e $\beta$ são os coeficientes de ponderação das componentes de segunda e terceira ordem, respectivamente, sendo que $\alpha, \beta \in[0,1]$.

2. Alto-falante não-linear com memória (NLML, do inglês Nonlinear and with Memory Loudspeaker).

Será utilizado o modelo do alto-falante representado por série de Volterra empregado no estudo de caso apresentado em [17, 18], os quais, por sua vez, fizeram uso de um modelo físico não-linear que se mostrou preciso na modelagem de um alto-falante real [19]. 
3. Alto-falante linear (LL, do inglês Linear Loudspeaker) dado pela parte linear do modelo em [19].

Daqui por diante referencia-se nesse trabalho o caso correspondente ao uso do primeiro modelo como: AEC com alto-falante não-linear sem memória - AEC(NLIL), o caso correspondente ao uso do segundo modelo como: AEC com alto-falante não-linear com memória - AEC(NLML) e o caso correspondente ao uso do terceiro modelo como: AEC com alto-falante linear $\operatorname{AEC}(\mathrm{LL})$.

\subsection{AEC pré-filtrado - PAEC}

Para fins de comparação considera-se também o caso em que a parte linear do modelo do alto-falante com memória é usada para filtrar a entrada do filtro adaptativo, denomina-se esse caso como AEC pré-filtrado.

Algumas aplicações de interesse do AEC pré-filtrado são listados a seguir:

1. AEC pré-filtrado aplicado ao alto-falante linear - LPAEC(LL).

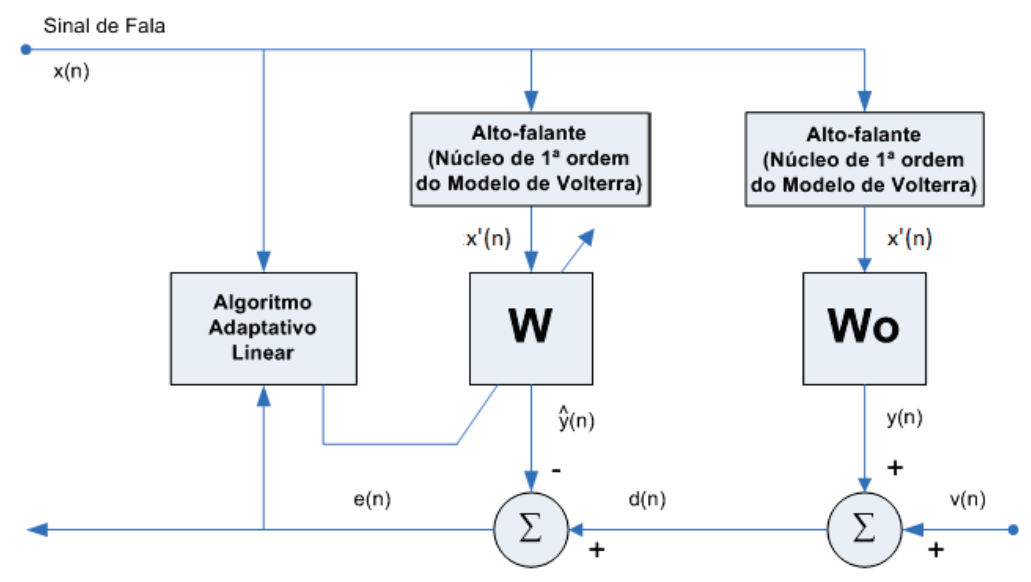

Figura 17: LPAEC(LL). 
2. AEC pré-filtrado aplicado ao alto-falante não-linear com memória LPAEC(NLML)

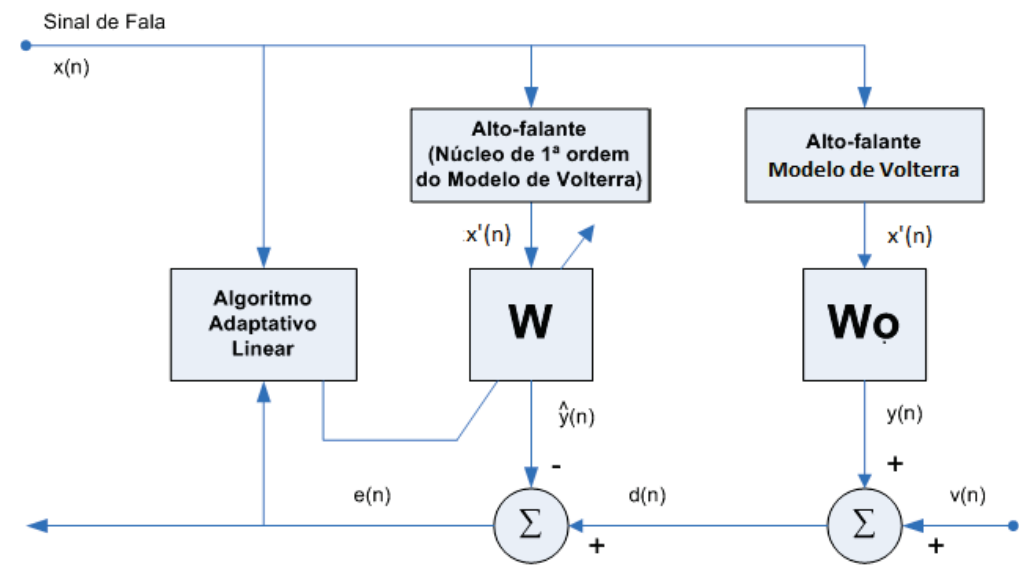

Figura 18: LPAEC(NLML).

\subsection{Análise da aplicação do modelo não-linear com memória}

O AEC(NLML) descrito na seção anterior utiliza um modelo não-linear que se mostrou preciso na modelagem de um alto-falante real [19] e, assim, se faz necessário o entendimento das características do modelo para que o comportamento de não-linearidade do alto-falante seja manifestado de forma mais significativa no sistema simulado.

Recorrendo à análise realizada em [19], nota-se que o modelo físico do altofalante se comporta como um filtro passa-baixas de frequência de corte igual a $500 \mathrm{~Hz}$ e que a frequência de amostragem usada para a obtenção do modelo de Volterra em [18] foi de $5 \mathrm{kHz}$.

Por consequência, conclui-se que existem três fatores importantes a serem tratados para correta simulação do AEC(NLML):

1. Frequência de amostragem do sinal de entrada. 
2. Magnitude do sinal de entrada.

3. Caracterização do sistema.

Com relação à frequência de amostragem do sinal de entrada foi necessário decimar o sinal de fala para obtenção de uma frequência de amostragem de $5 \mathrm{kHz}$, que é a mesma frequência utilizada para a obtenção do modelo não-linear de Volterra a ser utilizado no sistema. Como originalmente os sinais de entrada utilizados nas simulações têm frequência de amostragem de $16 \mathrm{kHz}$, foi empregada a decimação por um fator inteiro $3(\approx 16 / 5)$.

No que diz respeito à magnitude do sinal de entrada, um estudo mais minucioso do comportamento do modelo não-linear foi realizado através de simulações do AEC(NLML), sendo que o valor de variância necessário para se obter um efeito não-linear significativo foi definido com base na análise da precisão na aproximação do modelo do alto-falante pelo modelo de Volterra realizada em [18] e apresentado na Figura 19 na forma de índice de ajuste na aproximação entre os modelos. 


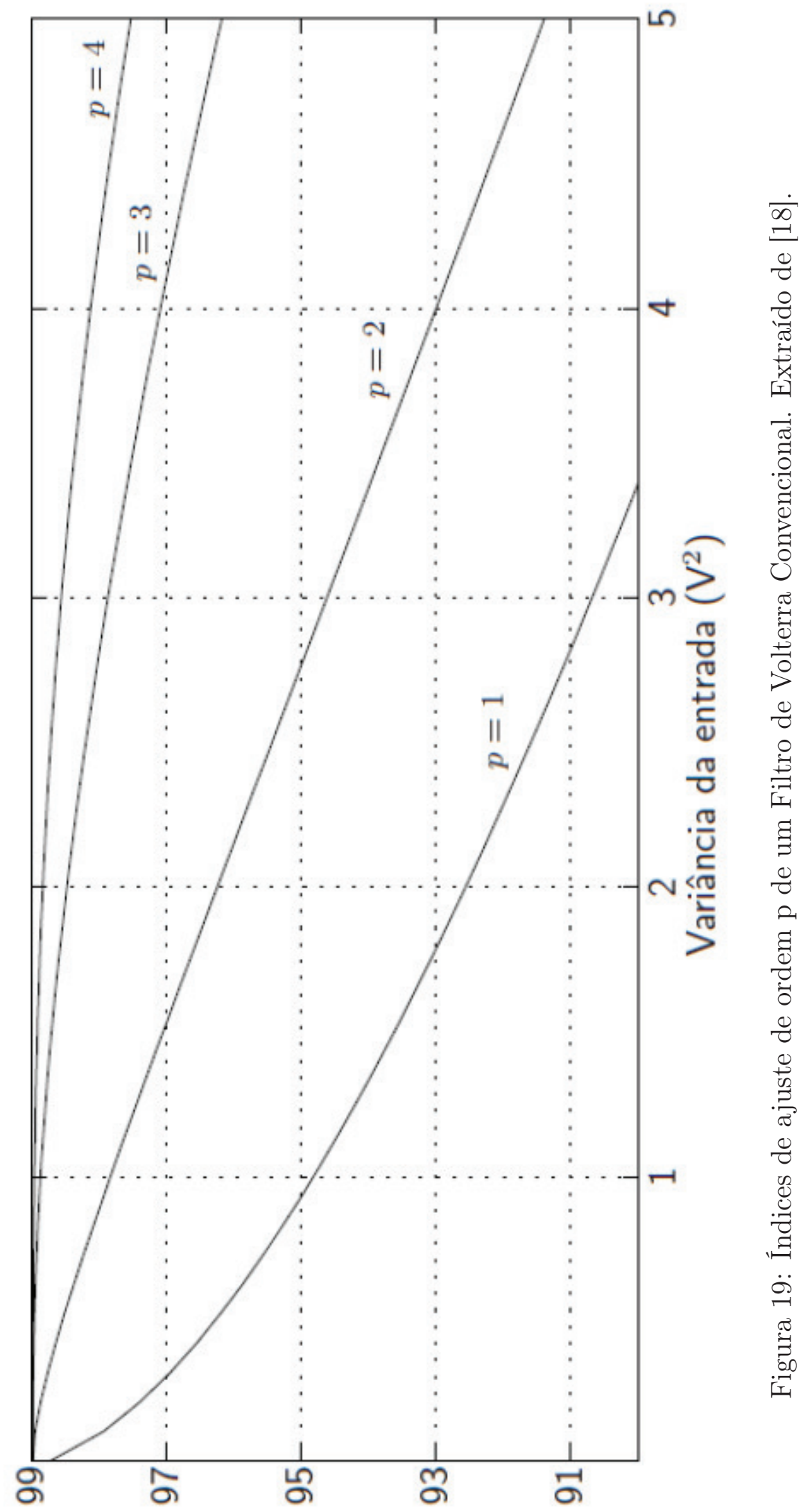

(\%) әғsnโе әр әэ!рu! 
Pela Figura 19 observa-se que sinais de entrada com variância menor ou igual a 5 resultam em uma precisão na representação do modelo do alto-falante superior a $91 \%$ para $p$ maior que 2 . Adicionalmente, verifica-se empiricamente o comportamento do cancelador de eco AEC(NLML) através de simulações de desempenho em termos do valor final de convergência do ERLE em função da variância do sinal de entrada considerando valores de 0 a 5 . Por fim, conclui-se que um sinal com variância igual a 5 desperta o comportamento não-linear do alto-falante com memória utilizado no presente trabalho. As simulações realizadas posteriomente nesse capítulo evidenciam tal fato atráves do desempenho dos sistemas simulados nessa condição.

Como mencionado anteriormente, o alto-falante reproduz apenas frequências até $500 \mathrm{~Hz}$ aproximadamente, tendo uma característica de filtro passa-baixas. Esse fato determinou que o sinal de entrada decimado fosse posteriormente filtrado por um filtro passa-baixas com frequência de corte igual a $500 \mathrm{~Hz}$. Dessa forma, o cancelamento de eco acústico considerado adiante corresponderia a uma parte de um sistema de cancelamento de eco acústico por sub-bandas.

\subsection{Simulação}

Os sistemas de cancelamento de eco acústico lineares descritos na seção anterior serão simulados através de scripts do software Matlab, que implementarão quatro algoritmos adaptativos lineares diferentes excitados por um sinal de fala de duração pré-determinada e uma resposta impulsiva real obtida experimentalmente em uma sala acusticamente isolada.

Os algoritmos adaptativos lineares a serem utilizados são os mesmos já descritos na seção 3 do capítulo 3 desse trabalho: LMS, NLMS, FBLMS e APA. Ou seja, LMS (algoritmo LMS padrão), NLMS (algoritmo LMS normalizado), 
FBLMS (algoritmo LMS implementado em blocos e no domínio da frequência) e APA (algoritmo de projeção afim). Destaca-se que os passos de adaptação desses algoritmos serão determinados empiricamente durante as simulações a fim de se obter o melhor desempenho em termos de ERLE para cada um deles.

O objetivo do uso desses algoritmos é analisar o comportamento dos mesmos quando empregados com a finalidade de cancelar o eco acústico nos casos de estudo de AEC já mencionados nesse capítulo.

Adicionalmente, considerando o AEC(NLIL), a análise do comportamento de cada algoritmo diante da variação do comportamento não-linear do alto-falante pode ser obtida variando-se os parâmetros $\alpha$ e $\beta$ da equação (4.4), ou seja, esse procedimento possibilita a análise de alto-falantes com características não-lineares diferentes. Assim, pretende-se investigar a sensibilidade de cada algoritmo com relação à intensidade da não-linearidade inserida pelo alto-falante no sistema LAEC.

Supõe-se que os comportamentos dos algoritmos sejam diferentes entre si e variáveis de acordo com cada caso estudado, haja vista a estrutura de cada algoritmo ser inerentemente diferente considerando alguns aspectos, tais como: processamento do sinal amostra-a-amostra ou bloco-a-bloco; processamento do sinal no domínio do tempo e/ou no domínio da frequência. 
O fluxograma genérico da simulação dos sistemas LAEC é apresentado abaixo:

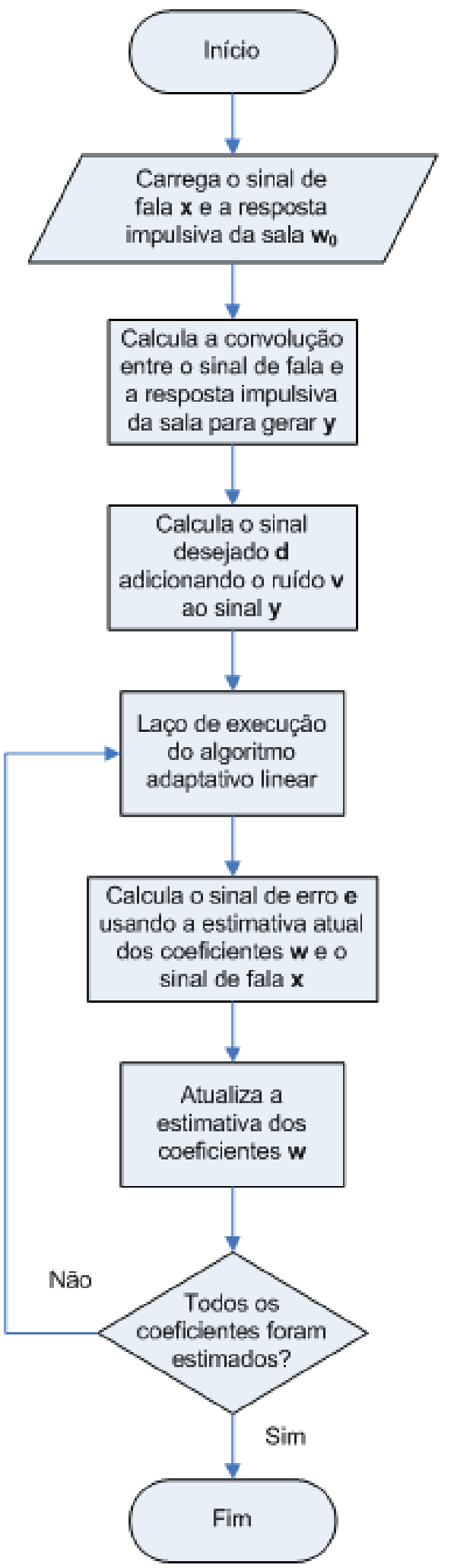

Figura 20: Fluxograma genérico dos sistemas LAEC simulados. 
Os sinais de fala utilizados no presente trabalho foram obtidos através da biblioteca digital de sinais de fala disponibilizada em [43]. Durante as simulações foram utilizados alguns sinais de fala de duração de 10s, de um mesmo locutor, que foram concatenados a fim de obter-se uma duração de 120 segundos do sinal. Experimentalmente, essa duração de tempo se mostrou suficiente para a obtenção da convergência dos canceladores de eco simulados.

A resposta impulsiva da sala usada nas simulações foi obtida através da base de dados SMARD [44]. Essa resposta foi estimada experimentalmente em um ambiente real isolado acusticamente e considerando uma configuração específica de dimensões da sala, posicionamento do alto-falante e microfone, temperatura, nível de pressão do som e taxa de amostragem. Assim, a referida resposta impulsiva da sala é uma estimativa que deve se aproximar muito da resposta impulsiva real do acoplamento alto-falante-microfone tendo em vista o método experimental minucioso que foi utilizado para sua obtenção.

Nas simulações desse capítulo, assim como no restante deste trabalho, utilizou-se 256 amostras da resposta impulsiva da sala para caracterizar o sistema $W_{o}$. Tal fato foi determinante para a escolha de um filtro adaptativo $W$ com 256 coeficientes, sendo que essa configuração do sistema adaptativo mostrou-se eficiente para cancelar o eco acústico em todas as simulações realizadas. Importante destacar que a resposta impulsiva da sala é considerada invariante no tempo neste trabalho, ou seja, o sistema $W_{o}$ não se modifica com o passar do tempo.

De modo geral, a presença de interferências captadas pelo microfone juntamente com o eco acústico prejudica a convergência de um AEC. Um exemplo disso é a interferência do sinal de fala local quando os dois usuários estão falando simultaneamente, porém este fator prejudicial não será considerado neste trabalho, ou seja, $s(n)=0$. 


\subsection{Resultados Obtidos}

Para a análise do grau de cancelamento do eco obtido através da simulação dos sistemas AEC(IL) e AEC(NLIL) utilizou-se como indicador de performance o parâmetro de melhoria da perda do retorno de eco (ERLE).

A Figura 21 revela o comportamento ERLE obtido por cada algoritmo em função do tempo em cada sistema simulado, sendo que utilizou-se em quase todos os casos simulados no presente trabalho $S N R=40 d B$, exceto nas simulações com variação de $\alpha$ e $\beta$ que serão mencionadas logo a seguir nessa seção. Analisando a equação (4.3) conclui-se que o ERLE tem valor máximo de 40dB definido pela SNR utilizada.

Além disso, excepcionalmente para essa simulação foi utilizado um sinal de fala com frequência de amostragem de $16 \mathrm{kHz}$ e duração de 1s concatenado para se obter 120s e no caso do AEC(NLIL) considerou-se $\alpha=\beta=0.8$. As exceções mencionadas se devem ao fato de que essa simulação tem por objetivo evidenciar a influência da não-linearidade no valor de limite superior do ERLE do cancelador de eco acústico, então defini-se o valor de $\mathrm{SNR}=120 \mathrm{~dB}$ e utiliza-se um sinal com menos intervalos de silêncio a fim de se obter menos variação nos valores de ERLE após a convergência de cada algoritmo. 


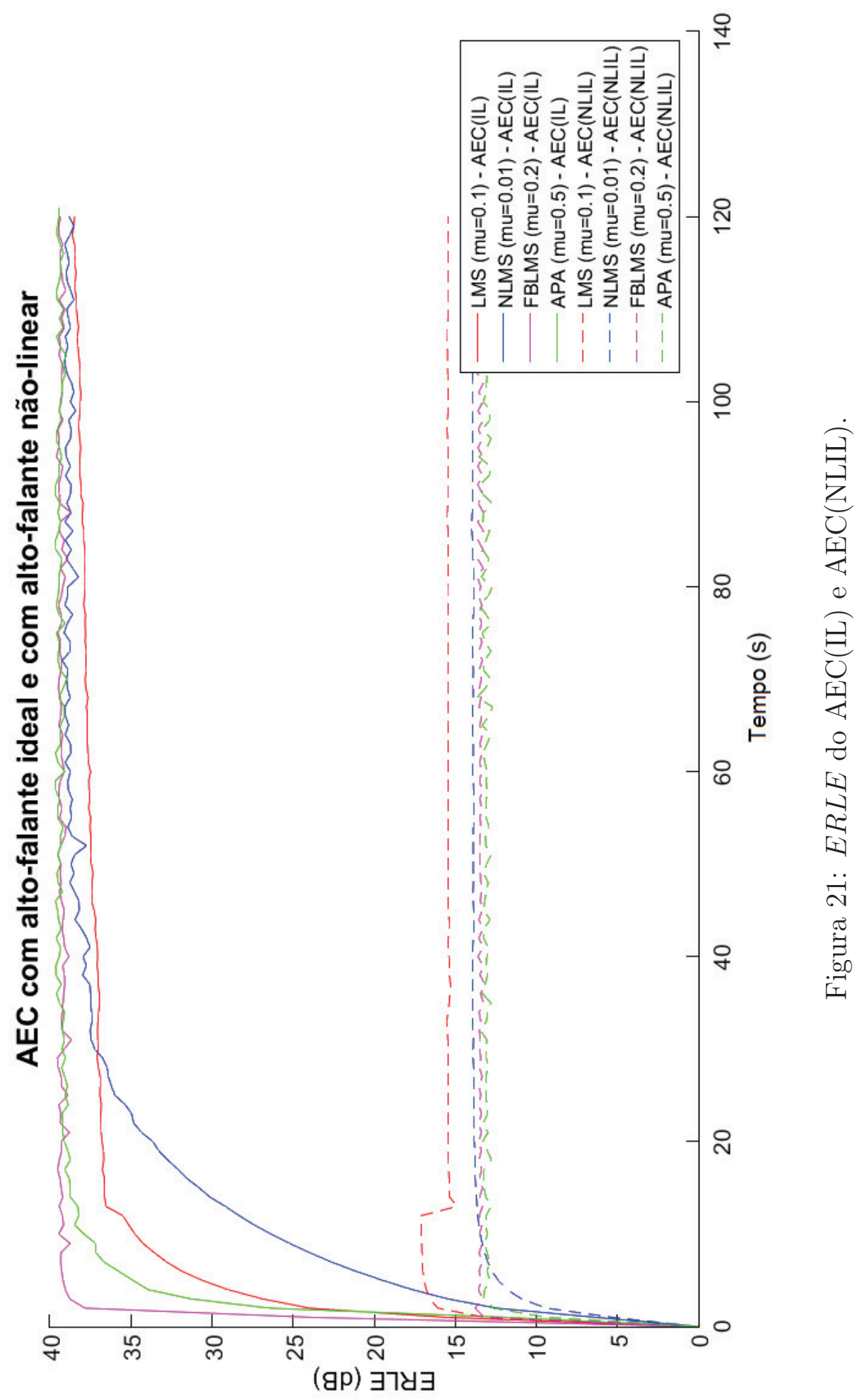


Dois experimentos foram realizados com o objetivo de estudar a sensibilidade de cada algoritmo empregado no LAEC frente a variações da não-linearidade do alto-falante. Em ambos os experimentos normalizou-se o sinal $x^{\prime}(n)$ e, excepcionalmente, utilizou-se um sinal de ruído da ordem de $10^{-12}$. Sendo assim, através da equação (4.3) conclui-se que o ERLE tem valor máximo de $110 \mathrm{~dB}$ definido pela SNR utilizada.

1. Experimento 1: Variação do parâmetro $\alpha$ no $A E C(N L I L)$

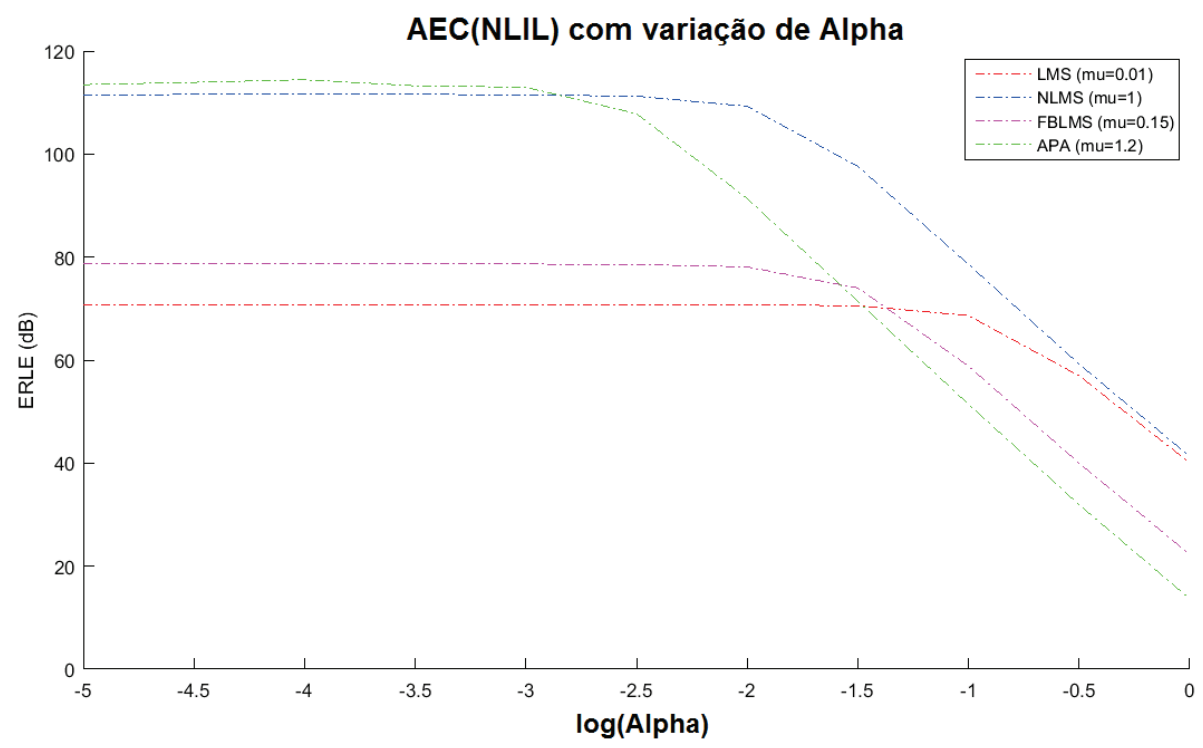

Figura 22: $E R L E$ do AEC(NLIL), $\beta=0$. 
2. Experimento 2: Variação do parâmetro $\beta$ no $A E C(N L I L)$

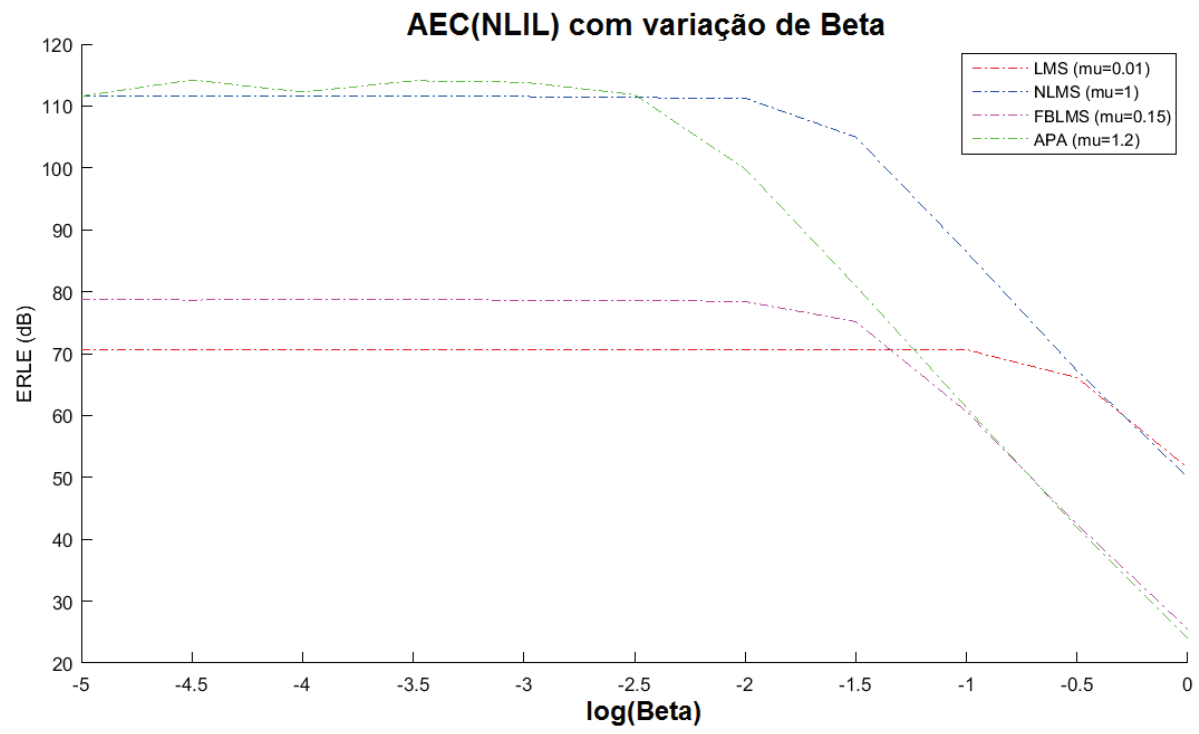

Figura 23: $E R L E$ do AEC(NLIL), $\alpha=0$.

Os quatro sistemas LAEC (AEC(IL), AEC(NLIL), AEC(NLML) e AEC(LL)) foram simulados conforme explicado anteriormente e os resultados foram analisados através de indicadores de desempenho mencionados na seção 4.2. Assim, para a análise do grau de cancelamento do eco obtido por cada um dos sistemas simulados utilizou-se como indicador de performance o parâmetro de melhoria da perda do retorno de eco (ERLE).

Para fins de comparação de desempenho dos sistemas propostos adotou-se a decimação do sinal de entrada, reduzindo sua frequência de amostragem de $16 \mathrm{kHz}$ para $5 \mathrm{kHz}$ em todos os sistemas simulados daqui em diante no presente trabalho.

Inicialmente, os sistemas $\mathrm{AEC}(\mathrm{IL})$ e $\mathrm{AEC}(\mathrm{LL})$ foram simulados e os resultados obtidos encontram-se na Figura 24.

Em seguida, os sistemas AEC(IL), AEC(LL), AEC(NLIL) e AEC(NLML) foram simulados e o ERLE obtido em cada caso é representado nos gráficos das figs. 25 e 26. 


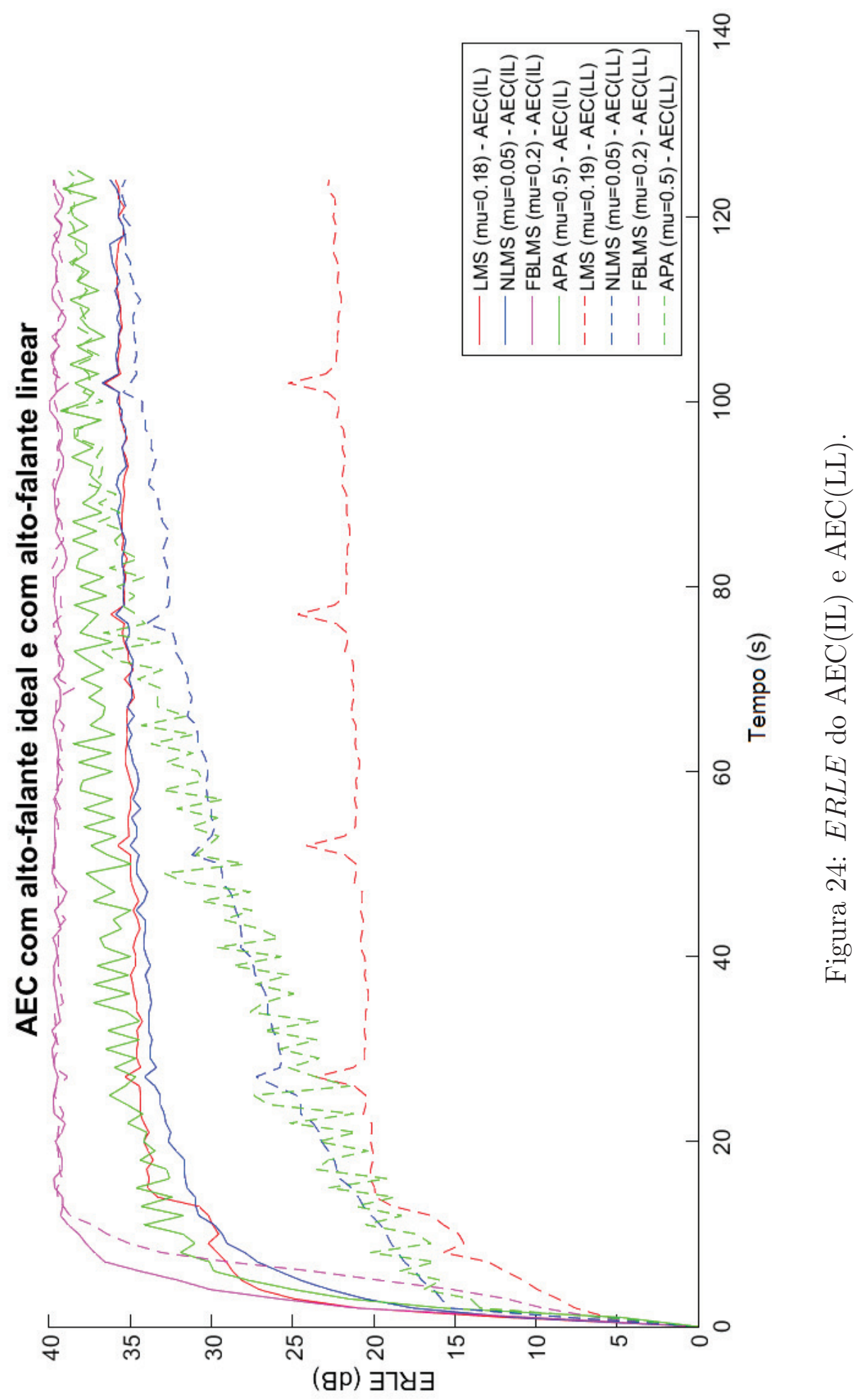




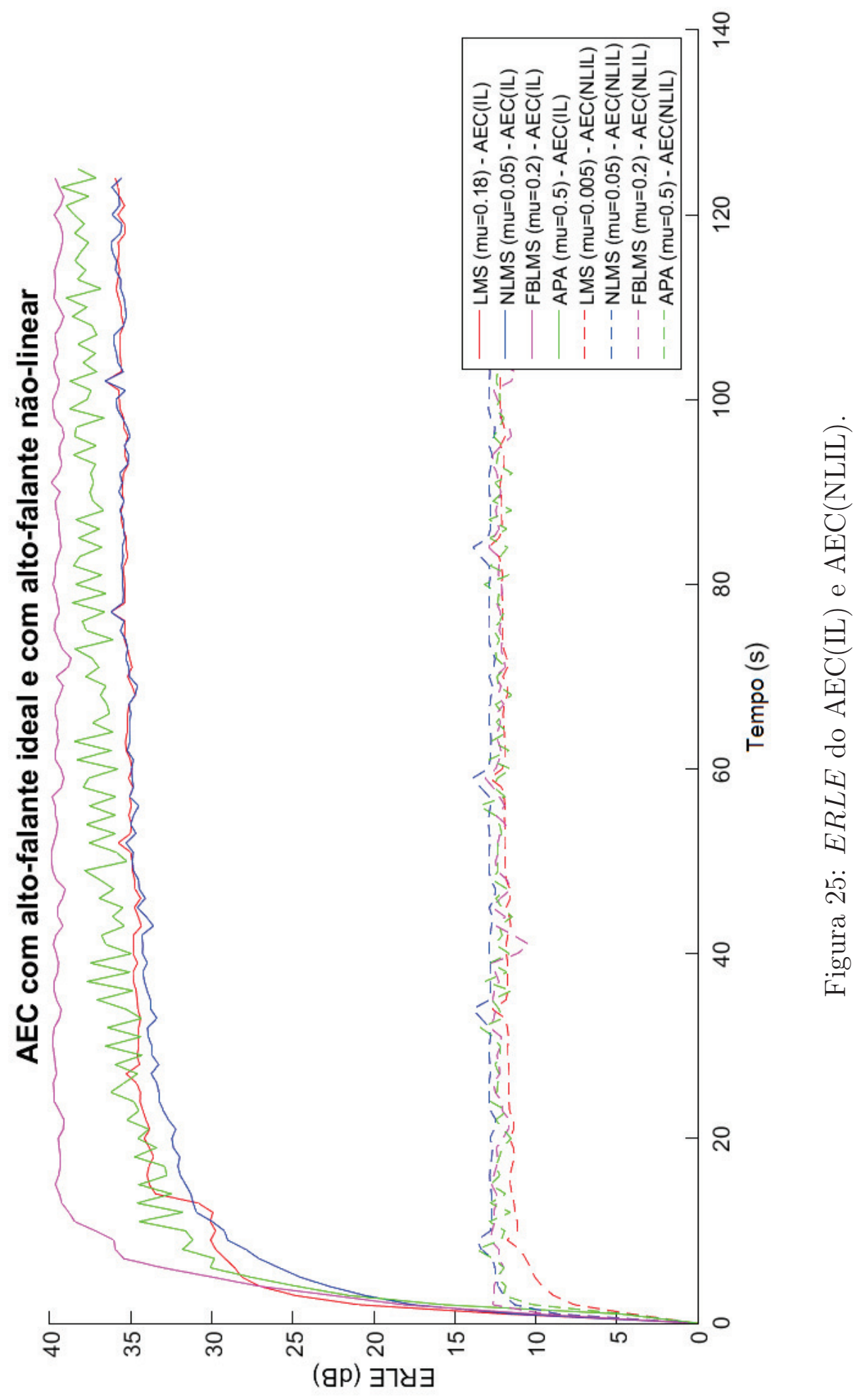




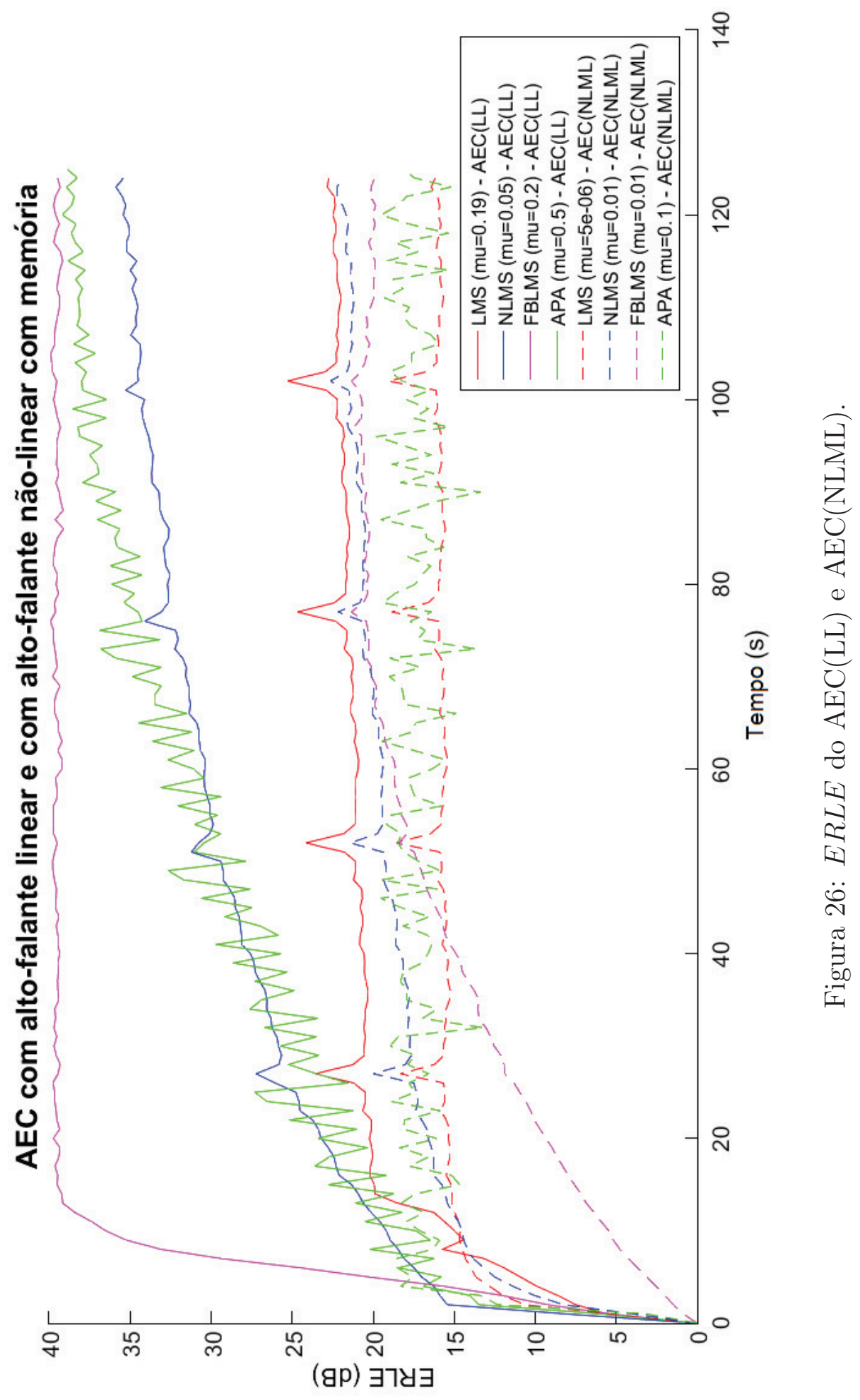




\subsection{Comparação de desempenho dos Algoritmos}

Inicialmente, o grau de cancelamento do eco dos sistemas AEC(IL) e AEC(NLIL) foi comparado através do gráfico 21, o qual revela uma grande diminuição do ERLE obtido no caso não-linear. No caso linear, a simulação do AEC(IL) mostra que todos os algoritmos alcançam aproximadamente o limite superior de ERLE imposto pela SNR e que o NLMS é o mais lento entre os algoritmos, pórem esse último fato não é relevante para as análise seguintes, pois o caso linear é um caso idealizado. Em particular, no caso não-linear, destaca-se o fato de que o algoritmo LMS nitidamente converge para uma solução diferente com relação aos demais algoritmos.

Considerando a simulação do AEC(NLIL) com variação do parâmetro $\alpha$ da equação (4.4), observa-se no gráfico 22 que todos os algoritmos se assemelham ao LMS quando se considera altas não-linearidades, ou seja, no intervalo de $\alpha=0,1$ a $\alpha=1$ os algoritmos têm aproximadamente a mesma taxa de variação do valor do ERLE.

Os algoritmos que apresentam melhor desempenho no caso de baixa nãolinearidade são o APA e o NLMS, ou seja, no intervalo de $\alpha=10^{-5}$ a $\alpha=0.1$ esses algoritmos apresentam valores de ERLE superiores aos dos algoritmos FBLMS e LMS.

Analisando a sensibilidade dos algoritmos à presença de não-linearidades notamos que o APA é o algoritmo mais afetado pelas altas não-linearidades, seguido do algoritmo FBLMS. Esses algoritmos tiveram a maior variação de valor do ERLE em termos absolutos ao se comparar o caso aproximadamente Linear $\left(\alpha=10^{-5}\right)$ e o caso Não-Linear mais severo $(\alpha=1)$.

Considerando a simulação do AEC(NLIL) com variação do parâmetro $\beta$ da equação (4.4), observa-se pelo gráfico 23 do ERLE que todas os comentários 
mencionados acima, tendo como base a variação do parâmetro $\alpha$, permanecem válidos.

Para fins de comparação entre os sistemas, todas as análises de resultados de simulações mencionadas daqui por diante nesse texto consideram a dizimação do sinal de entrada de $16 \mathrm{KHz}$ para $5 \mathrm{KHz}$, já que essa é a frequência utilizada para a obtenção do modelo não-linear de Volterra adotado no presente trabalho.

Continuando a análise, o grau de cancelamento do eco dos sistemas AEC(IL) e AEC(LL) foi comparado através do gráfico 24, o qual revelou velocidade de convergência menor no caso do AEC(LL) para todos os algoritmos adaptativos simulados. Porém, os valores do ERLE após a convergência dos algoritmos foram aproximadamente os mesmos em todos os casos.

Considerando as não-linearidades do alto-falante, o grau de cancelamento do eco é reduzido significativamente tanto no AEC(NLIL) como no AEC(NLML), como podemos observar pelos gráficos 25 e 26, respectivamente. Nesses gráficos os valores de ERLE obtidos nas simulações dos sistemas AEC(IL) e AEC(LL) são utilizados como referências para as análises das simulações dos outros sistemas.

Os resultados obtidos nas simulações realizadas mostram que as nãolinearidades do alto-falante não podem ser desprezadas, pois prejudicam consideralvelmente o desempenho do LAEC, havendo portanto forte motivação para empregar cancelamento de eco acústico não-linear.

\subsection{Conclusões}

$\mathrm{Na}$ presença de não-linearidades todos os algoritmos adaptativos lineares apresentaram pior desempenho de ERLE tanto na simulação do AEC(NLIL) como dos sistemas AEC(NLML) e LPAEC(NLML), tendo como referência os valores de ERLE obtidos na simulação dos sistemas AEC(IL) e AEC(LL). Esse compor- 
tamento já era esperado, pois, como sabemos, as não-linearidades degradam a performance dos canceladores de eco adaptativos lineares.

Os algoritmos em bloco e no domínio da frequência são mais prejudicados pelo aumento da não-linearidade do sistema, como podemos notar pela análise dos valores do ERLE dos algoritmos APA e FBLMS presentes nos gráficos 22 e 23, que consideram variações dos parâmetros $\alpha$ e $\beta$ do AEC(NLIL), respectivamente. Os algoritmos que apresentam melhor performance no caso de baixa não linearidade são o APA e o NLMS; e o algoritmo que apresenta pior desempenho nessa situação é o FBLMS, pois apresenta valor de ERLE muito pequeno, até mesmo considerando o menor valor de não-linearidade, ou seja, o caso quase linear.

O uso de um modelo realista para a representação do alto-falante revela a incapacidade do cancelador de eco adaptativo linear em identificar o filtro real $\mathbf{W}_{0}$ com precisão, esse é o caso representado pelo AEC(NLML) que utiliza o modelo do alto-falante usando núcleos de Volterra. 


\section{SISTEMA PROPOSTO}

Na literatura encontramos alguns trabalhos que têm por objetivo investigar o comportamento dos canceladores de eco na presença de não-linearidades do alto-falante através da modificação do AEC(IL). Essa modificação é obtida geralmente através da inclusão de um pré-processador não-linear (NLP) antes do filtro adaptativo, ou seja, cascateando o sistema adaptativo da forma apresentada pela Figura 32 .

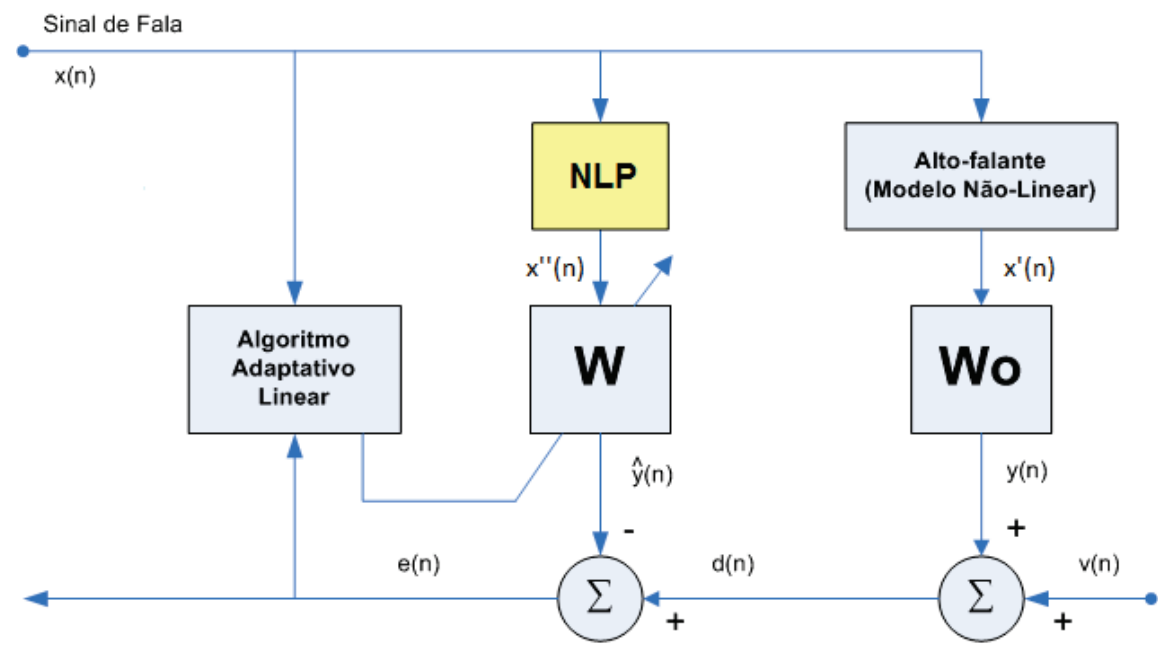

Figura 27: Cancelador de eco acústico com NLP.

Seguindo a mesma metodologia, o presente trabalho propõe um sistema NLAEC capaz de melhorar o desempenho do AEC na presença de nãolinearidades provenientes do alto-falante. O uso desse modelo foi motivado pelo trabalho realizado em [15], no qual uma estrutura em cascata similar foi proposta, sendo que o filtro de Volterra utilizado como pré-processador não-linear 
tinha seus núcleos definidos adaptativamente.

Destaca-se que nesse capítulo se utiliza a função ODE15s do Matlab para resolução das equações de espaço de estados do modelo do alto-falante a fim de se obter uma maior precisão na representação do alto-falante modelado por [19]. Esse método de resolução das EDO's se mostrou mais eficiente do que as funções ODE45 e ODE113 durante as execuções das simulações do presente trabalho. Isso pode ser explicado pela capacidade da função $O D E 15 \mathrm{~s}$ em resolver equações de modelos com característica de stiffness.

\subsection{Efeito da Adaptação}

Analisando o efeito do processo de adaptação do cancelador linear de eco acústico, é esperado que o filtro adaptativo ajude a reduzir o efeito da imprecisão do modelo. Sua resposta ótima é $\mathbf{R}^{-1} \mathbf{p}$, onde $\mathbf{R}$ é a matriz de autocorrelação da entrada e p é o vetor de correlação cruzada entre a entrada e o sinal desejado. Uma vez que o sinal desejado é a convolução do sinal de entrada com a resposta impulsiva da sala $h$ e considerando que o filtro adaptativo tem pelo menos o comprimento de $h$, então a resposta ótima é o próprio $h$.

Isso valeria se o modelo do alto-falante fosse exato. Porém, na prática, o modelo não é exato e o sinal desejado é uma função não-linear do sinal de entrada do filtro adaptativo com alto grau de complexidade . Neste caso, a resposta ótima não é necessariamente igual a $h$ e, além disso, ela depende da amplitude do sinal de entrada, dada a não-linearidade envolvida.

O efeito de adaptação do sistema proposto pode ser constatado através da análise do sistema na condição em que não existe adição de ruído ao processo adaptativo, ou seja, o resultado da adaptação se deve apenas à ação do algoritmo adaptativo sobre o sinal de entrada. Além disso, é necessário avaliar o erro re- 
sultante da imprecisão do modelo não-linear, empregado como bloco NLP, em representar o modelo do alto-falante, assim defini-se o indicador Erro de Aproximação Normalizado (NAE, do inglês Normalized Approximation Error) pela expressão

$$
\boldsymbol{N} \boldsymbol{A} \boldsymbol{E}(d B)=10 \log _{10}\left(\frac{\sigma_{y_{1}^{2}}^{2}}{\sigma_{e_{a}^{2}}}\right)
$$

onde $\sigma_{y 1}^{2}$ e $\sigma_{e}^{2}$ referem-se às variâncias do sinal de saída do modelo do alto-falante e do erro de aproximação entre os modelos, $y_{1}(n)$ e $e_{a}(n)$, respectivamente.

Sendo que

$$
e_{a}(n)=y_{1}(n)-y_{2}(n)
$$

onde $y_{2}$ é a saída do modelo não-linear empregado como bloco NLP.

O indicador NAE mostra o quanto a saída de um modelo não-linear empregado como bloco NLP se aproxima da saída do modelo do alto-falante durante o processo de adaptação, ou seja, quanto maior for o valor em decibéis de NAE melhor será a precisão do modelo não-linear na representação do modelo do altofalante.

\subsection{Pré-Processamento Não-Linear}

O sistema em cascata proposto, NLPAEC(NLML), consiste de um estágio de pré-processamento não-linear implementado através do modelo não-linear do alto-falante obtido em [17, 18], já utilizado no capítulo anterior na simulação do AEC(NLML) e que se mostrou preciso experimentalmente.

Na prática, o sistema de viva-voz completo incluiria o alto-falante e o sistema AEC, de forma que o fabricante incorporaria o modelo apropriado de alto-falante 
no AEC.

\subsubsection{Sistema Proposto Ideal}

A simulação do AEC(NLML) realizada no capítulo anterior será refeita considerando a inclusão do bloco NLP no modelo proposto, implementado pelo modelo não-linear do alto-falante. O diagrama da Figura 28 descreve o NLPAEC(NLML) proposto no presente trabalho:

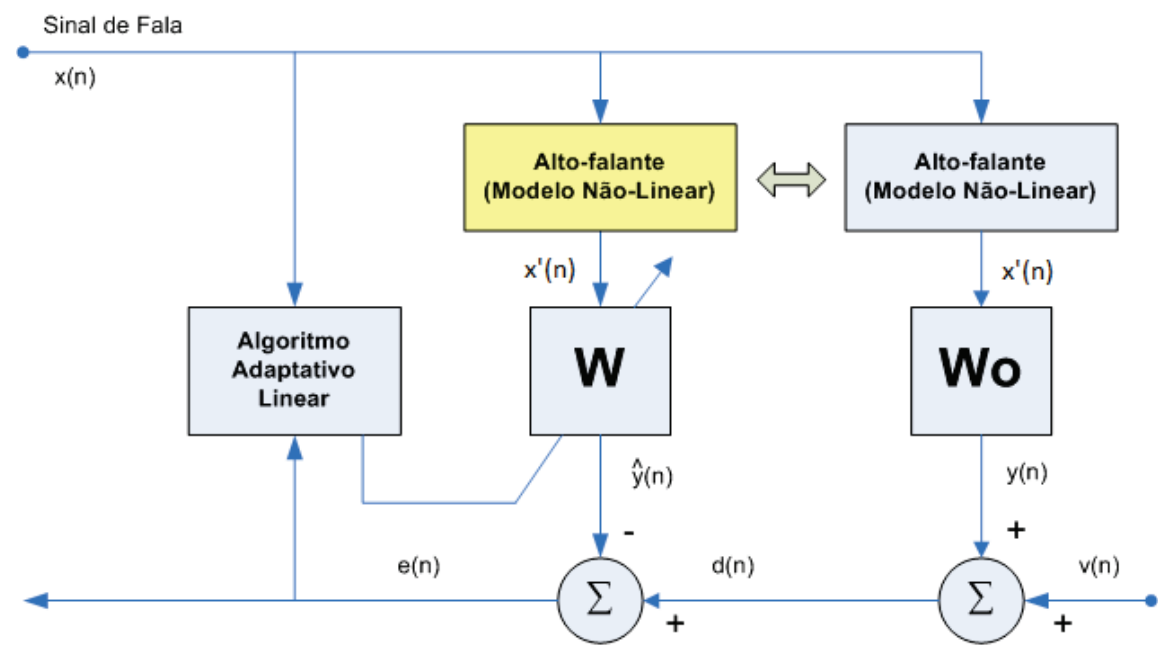

Figura 28: NLPAEC(NLML).

A adoção do próprio modelo do alto-falante como bloco de pré-processamento não-linear nessa simulação tem por objetivo evidenciar que o modelo proposto possui idealmente a mesma capacidade de cancelar o eco acústico que o sistema AEC(IL), pois segue que em ambos os casos o filtro adaptativo deve tender à resposta impulsiva acústica da sala.

\subsubsection{Resultados Obtidos}

O NLPAEC(NLML) foi simulado conforme explicado anteriormente e os resultados foram analisados através de indicadores dos desempenho mencionados na seção 4.2 . 
Para a análise do grau de cancelamento do eco obtido pelo sistema simulado utilizou-se como indicador de performance o parâmetro de melhoria da perda do retorno de eco (ERLE). Os gráficos das Figuras 29,30 e 31 revelam o comportamento ERLE obtido com as simulações dos sistemas NLPAEC(NLML), AEC(NLML), LPAEC(NLML) e AEC(IL). 


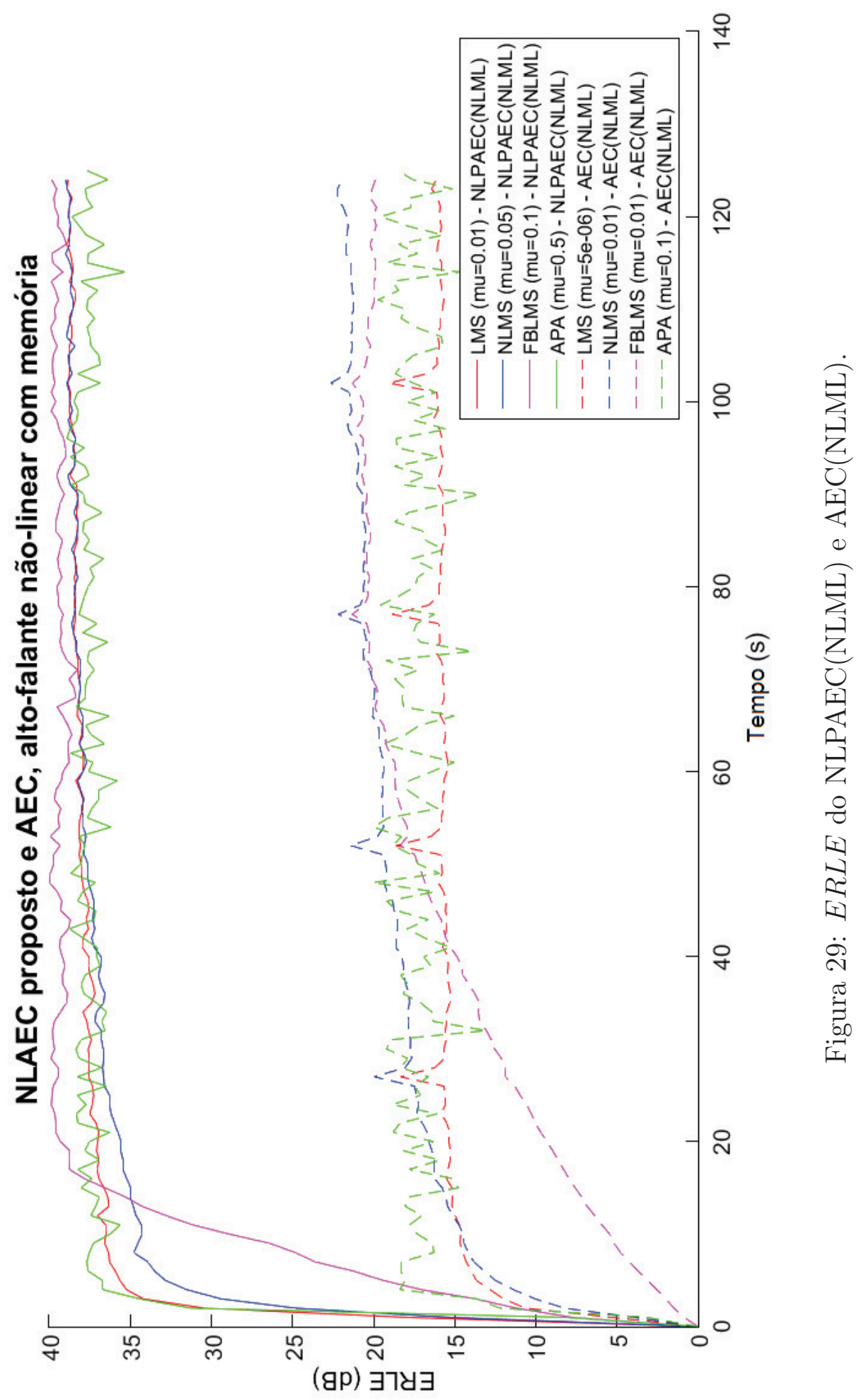




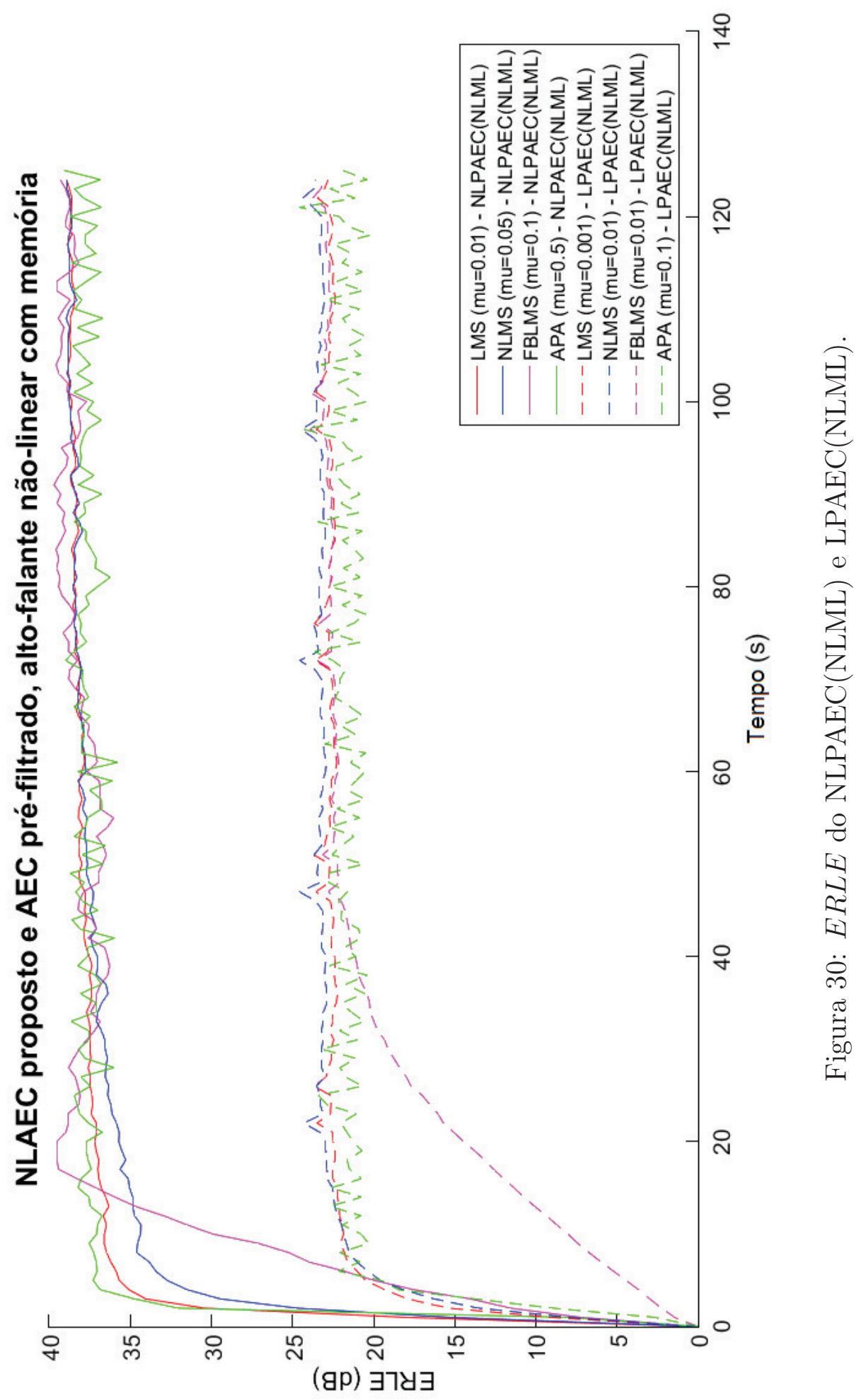




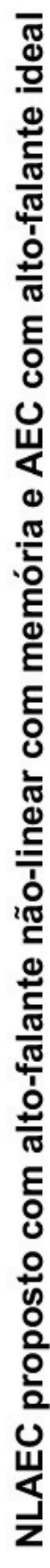

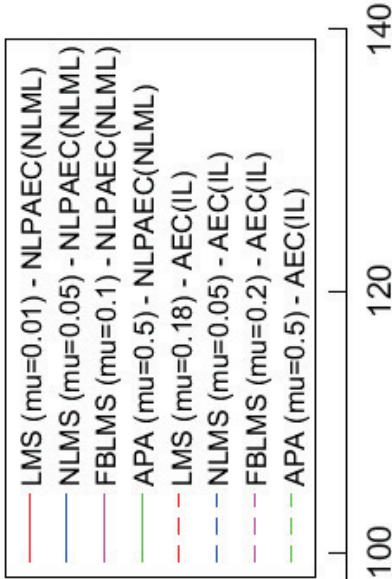

움

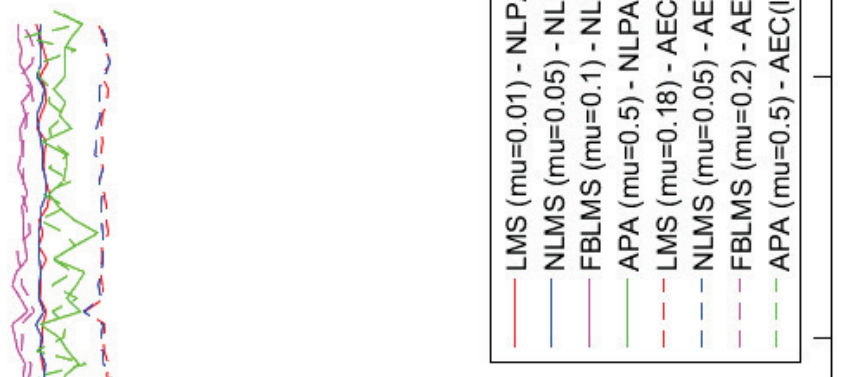

ํำ

음

臽

有?
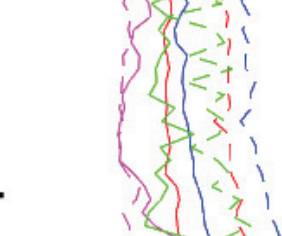

$1 \times \frac{1}{1}$

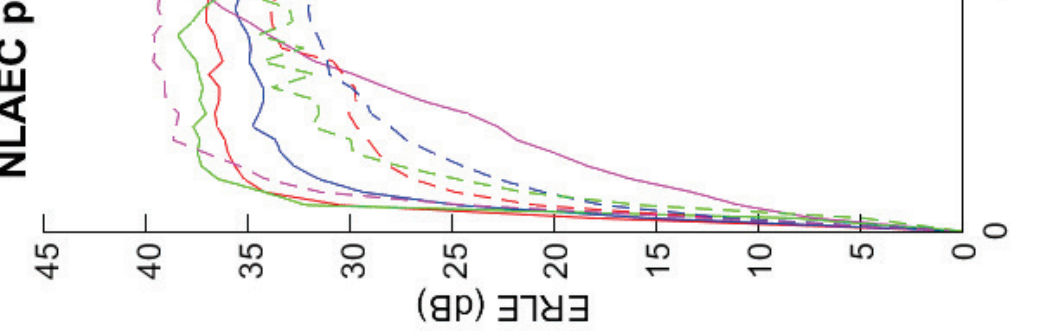




\subsubsection{Comparação de desempenho dos Algoritmos}

Utilizando um pré-processador não-linear (NLP) como descrito na seção 5.2, o grau de cancelamento do eco obtido pelo NLPAEC(NLML) é aumentado significativamente em relação ao AEC(NLML) e LPAEC(NLML), conforme podemos notar analisando os gráficos 29 e 30.

Na Figura 31 comparam-se o NLPAEC(NLML) e o AEC(IL). Como o bloco de pré-processamento não-linear adotado é, idealmente, o próprio modelo do altofalante, segue que em ambos os casos o filtro adaptativo deve tender à resposta impulsiva acústica.

Analisando o trecho inicial das curvas, é de interesse observar que, com exceção do FBLMS, a velocidade de convergência dos algoritmos testados é maior no caso do sistema NLPAEC(NLML) do que no caso do sistema AEC(IL). Uma possível explicação para esse comportamento é que o pré-processamento melhora o condicionamento do sinal de entrada do filtro adaptativo.

Comparando agora o trecho final das curvas, observa-se que no caso do LMS e do NLMS o AEC com pré-processamento não-linear também apresenta um ERLE em regime um pouco maior do que no caso de AEC convencional com alto-falante ideal. Esse fato sugere que em casos de altas não-linearidades os algoritmos mais apropriados para utilização em canceladores de eco seriam o NLMS e LMS.

\subsubsection{Conclusões}

O NLPAEC(NLML) apresentou melhora significativa de desempenho em termos de grau de cancelamento de eco ERLE em comparação com os sistemas AEC(NLML) e LPAEC(NLML). De modo geral, houve um pequeno aumento na velocidade de convergência dos algoritmos testados usando o sistema em cascata proposto, com exceção do algoritmo FBLMS. Provavelmente, a melhora do 
condicionamento do sinal de entrada do filtro adaptativo propiciada pelo préprocessamento é a explicação disso.

Assim, o NLPAEC(NLML), que utiliza uma etapa de pré-processamento nãolinear (NLP) anterior ao filtro adaptativo, apresentou melhor desempenho considerando os indicadores ERLE e Velocidade de Convergência.

Conclui-se que o NLPAEC(NLML) serve como um modelo de sistema capaz de cancelar o eco acústico na presença de altas não-linearidades. Sendo assim, na primeira parte do presente trabalho foi proposto um método eficiente para obtenção de um melhor desempenho dos canceladores de eco.

\subsection{Compromisso entre Desempenho e Complexi- dade Computacional}

No presente trabalho pretende-se determinar qual das possíveis estruturas de implementação do bloco de NLP resulta em um melhor compromisso entre carga computacional e precisão na aproximação do alto-falante, baseando-se na performance do NLPAEC(NLML) que se utiliza de tais estruturas.

Para representação do modelo do alto-falante com maior precisão foi adotado o método de resolução do Matlab ODE15s, sendo que o cancelador de eco nãolinear proposto passa a ter a configuração descrita pelo diagrama da Figura 32.

Nessa seção primeiramente analisam-se os resultados das simulações de cancelamento de eco acústico utilizando o esquema proposto considerando as diversas estruturas alternativas não-lineares empregadas como bloco NLP. Além disso, dados de complexidade estrutural e carga computacional de cada modelo são apresentados, permitindo a determinação do melhor compromisso entre desempenho e carga computacional. 


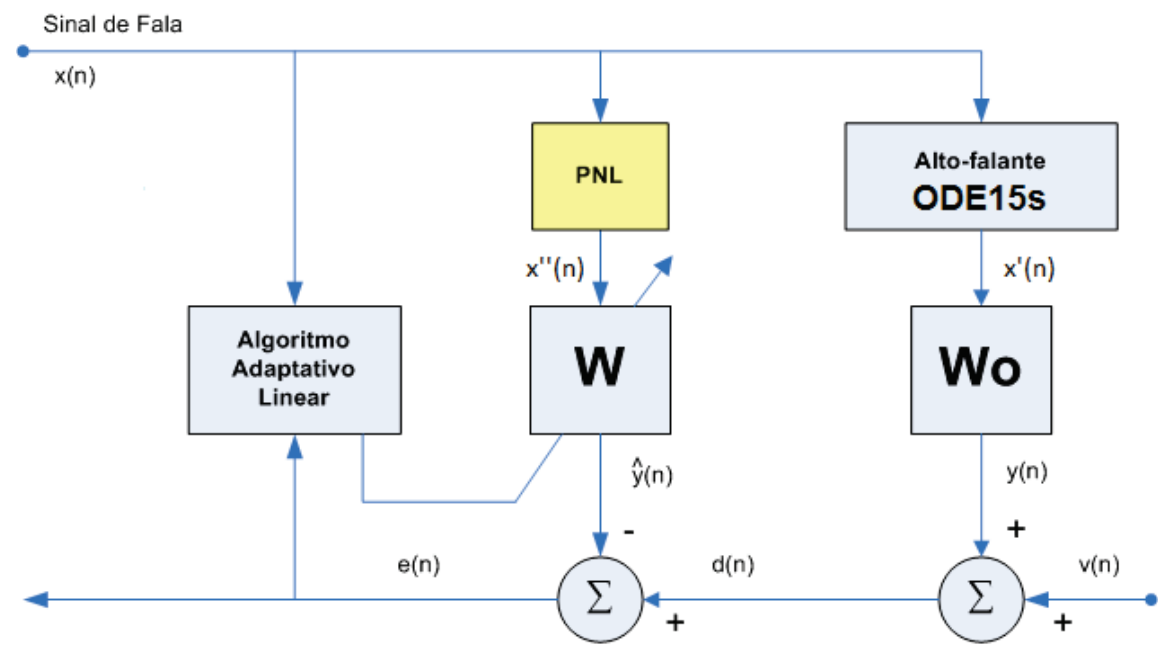

Figura 32: Cancelador de eco não-linear proposto - NLPAEC(NLML).

\subsubsection{Desempenho do Sistema Proposto}

Nas simulações descritas a seguir foram analisadas opções de implementação do bloco de NLP conforme descrição abaixo:

1. Resolução das equações de espaço de estados por $O D E 15 s$.

2. Filtro de Volterra Convencional de $1^{\mathrm{a}}$ ordem.

3. Filtro de Volterra Convencional de $2^{\mathrm{a}}$ ordem.

4. Filtro de Volterra Convencional de $3^{\mathrm{a}}$ ordem.

5. Modelo PARAFAC-Volterra de $2^{\mathrm{a}}$ ordem.

6. Modelo PARAFAC-Volterra de $3^{\mathrm{a}}$ ordem.

7. Filtro de Volterra Esparso Interpolado de $2^{\mathrm{a}}$ ordem.

8. Filtro de Volterra Esparso Interpolado de $3^{\mathrm{a}}$ ordem.

9. Discretização de Euler das equações de espaço de estados.

10. Discretização de Euler do Sistema Bilinear do modelo físico. 
Para análise de desempenho do cancelador de eco acústico não-linear proposto, NLPAEC(NLML), foi utilizado o indicador ERLE (Echo Return Loss Enhancement) através da equação (4.3). Nessa seção adota-se como critério de classificação do desempenho do cancelador de eco acústico em termos de ERLE os seguintes valores e rótulos: $\geq 35 \mathrm{~dB}$ - Excelente; $\geq 33 \mathrm{~dB}$ - Ótimo; $\geq 30 \mathrm{~dB}$ Bom; $\leq 29 \mathrm{~dB}$ - Ruim. Além disso, vale destacar que esses valores de ERLE considerados na classificação correspondem aos valores máximos de pico do ERLE alcançados por cada sistema em cada uma das simulações.

A análise de desempenho dos algoritmos adaptativos lineares realizada na seção 5.2 mostrou que os algoritmos LMS e NLMS tiveram melhor desempenho em termos de ERLE em caso de altas não-linearidades. Assim, tais algoritmos são os mais apropriados para utilização em canceladores de eco não-lineares.

Na seção 5.2, o bloco NLP do sistema proposto consistia do próprio modelo do alto-falante. Portanto, essa configuração se tratava de um caso ideal de cancelamento de eco acústico no qual o filtro adaptativo converge para a resposta acústica da sala. Em contrapartida, as simulações dessa seção consideram as estruturas não-lineares descritas no Capítulo 3 como alternativas de implementação do bloco NLP, logo não se tratam de casos ideais. Tal fato é determinante na escolha do algoritmo NLMS para a realização dessas simulações, pois o NLMS é mais robusto a variações de potência do sinal de entrada.

As simulações consideraram uma duração de 10s do sinal de fala de um locutor masculino obtido através da biblioteca TIMIT [43]. Além disso, o valor de intervalo de tempo para a análise do ERLE foi escolhido como 0,1s, definindo a janela de observação gráfica do indicador ERLE; a variância do sinal de entrada foi considerada igual à 5 , com o objetivo de despertar um comportamento altamente não-linear do modelo do alto-falante; e, adicionalmente, um filtro passa-baixas com frequência de corte igual a $500 \mathrm{~Hz}$ foi aplicado ao sinal de entrada limitando 
seu espectro à banda passante do modelo do alto-falante, o qual se comporta como um woofer, conforme explicado na seção 3.1.3.

As simulações dos canceladores de eco acústico foram agrupadas em 4 setups de análise, sendo que cada setup define quais estruturas não-lineares serão utilizadas como bloco NLP. Os setups são descritos abaixo:

1. Setup 1: ODE15s e Filtro de Volterra Convencional (1 $1^{\mathrm{a}}, 2^{\mathrm{a}}$ e $3^{\mathrm{a}}$ ordens).

2. Setup 2: Filtro de Volterra Convencional ( $2^{\mathrm{a}}$ e $3^{\mathrm{a}}$ ordens) e Modelo PARAFAC-Volterra ( $2^{\mathrm{a}}$ e $3^{\mathrm{a}}$ ordens).

3. Setup 3: Modelo PARAFAC-Volterra ( $2^{\mathrm{a}}$ e $3^{\mathrm{a}}$ ordens) e Filtro de Volterra Esparso Interpolado ( $2^{\mathrm{a}}$ e $3^{\mathrm{a}}$ ordens $)$

4. Setup 4: Modelo PARAFAC-Volterra ( $2^{\mathrm{a}}$ e $3^{\mathrm{a}}$ ordens) e Discretização de Euler (equação de espaço de estados e sistema bilinear).

Cada um dos setups descritos acima foi simulado em 4 cenários distintos, nos quais a duração da janela de observação foi modificada (1s ou $0.1 \mathrm{~s}$ ), o sinal de ruído no sistema adaptativo proposto foi manipulado (removido ou $\mathrm{SNR}=40 \mathrm{~dB}$ ) e o Erro de Aproximação Normalizado (NAE) dos modelos foi apresentado. Os cenários considerados são descritos abaixo:

1. Cenário 1: ERLE do Sistema Proposto com Janela=1s e com $\mathrm{SNR}=40 \mathrm{~dB}$.

2. Cenario 2: ERLE do Sistema Proposto com Janela=0.1s e com $\mathrm{SNR}=40 \mathrm{~dB}$.

3. Cenário 3: ERLE do Sistema Proposto com Janela=0.1s e sem Ruído.

4. Cenário 4: NAE do Sistema Proposto com Janela=0.1s.

O Cenário 1 trata do Sistema Proposto originalmente com adição de ruído, $\mathrm{SNR}=40 \mathrm{~dB}$, e janela de observação de $1 \mathrm{~s}$. A janela de observação é modificada 
de 1s para 0.1s no Cenário 2 a fim de propiciar uma análise mais detalhada dos valores alcançados pelo ERLE. Os Cenários 3 e 4 oferecem condições ideais para a análise do efeito de adaptação mencionada na seção 5.1, pois no Cenário 3 não existe adição de ruído ao processo adaptativo e no Cenário 4 o Erro de Aproximação Normalizado (NAE) é apresentado. 


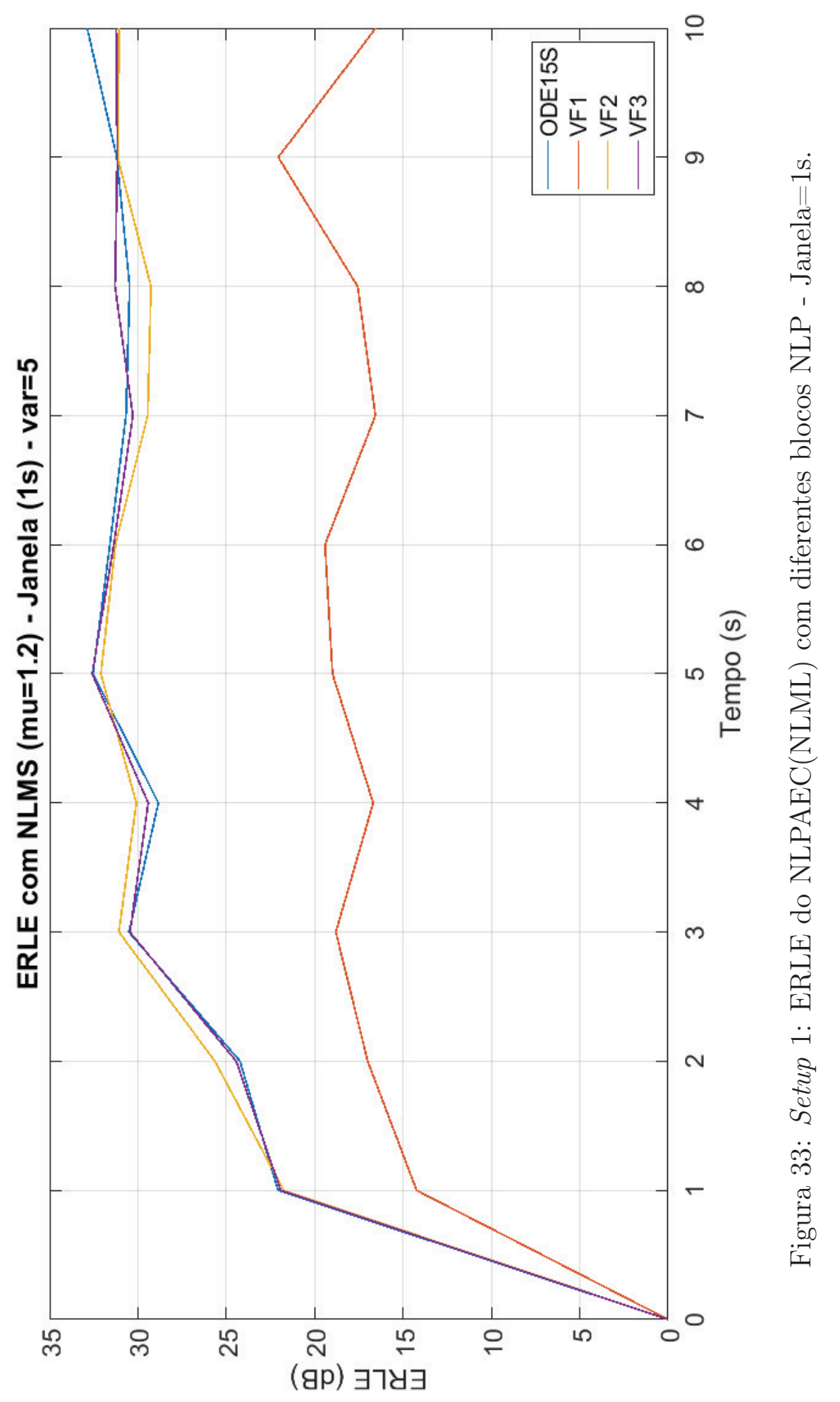




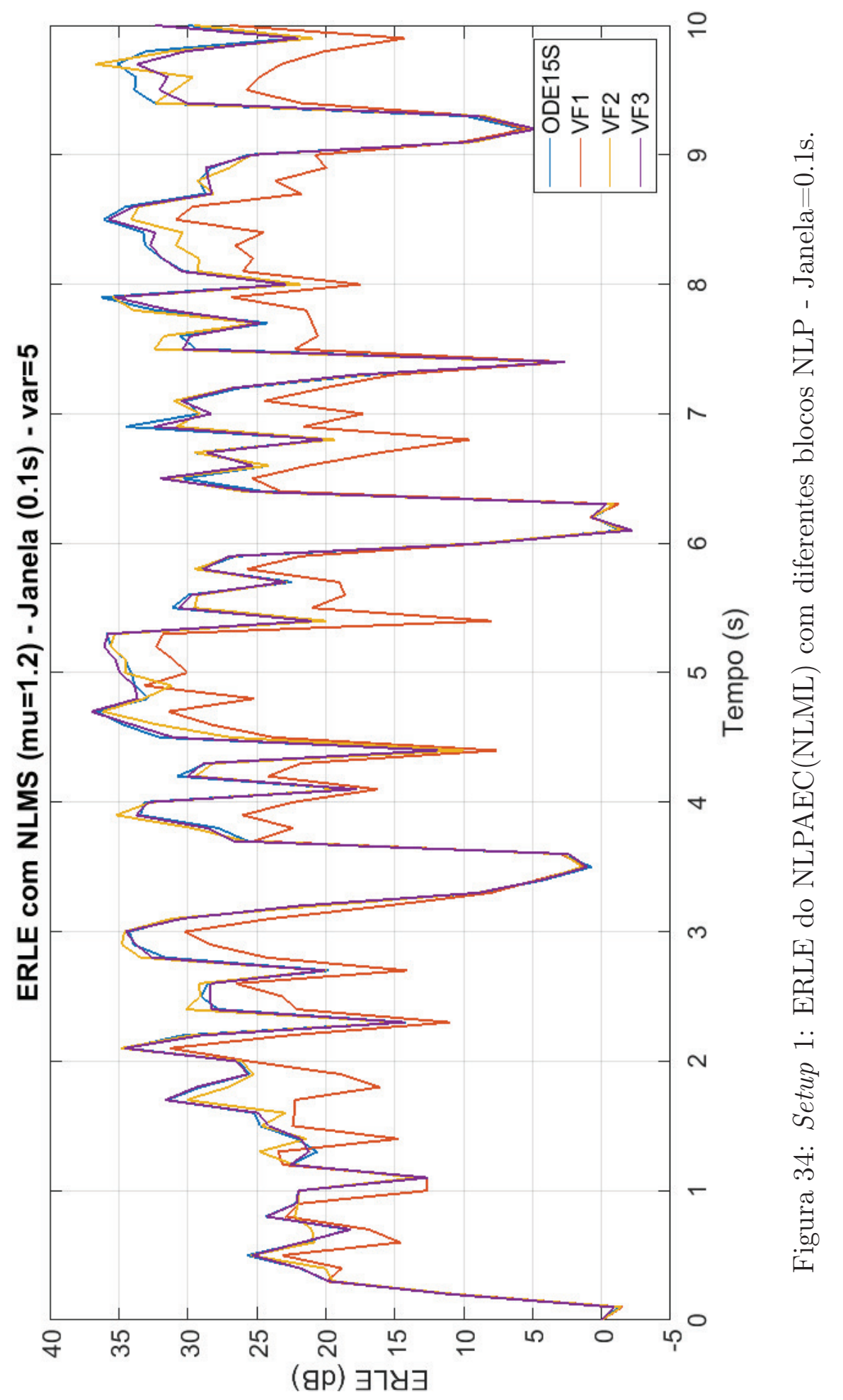




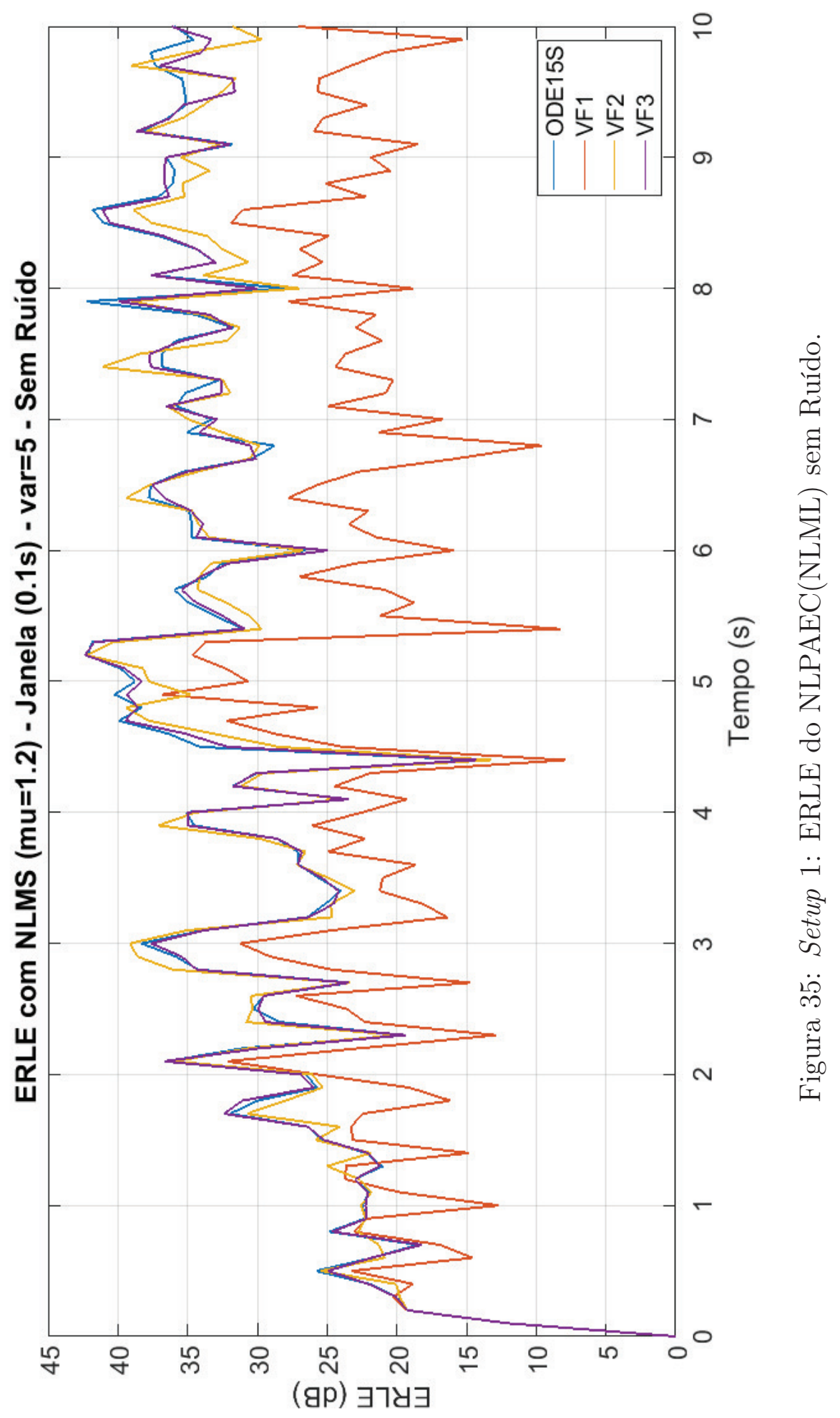




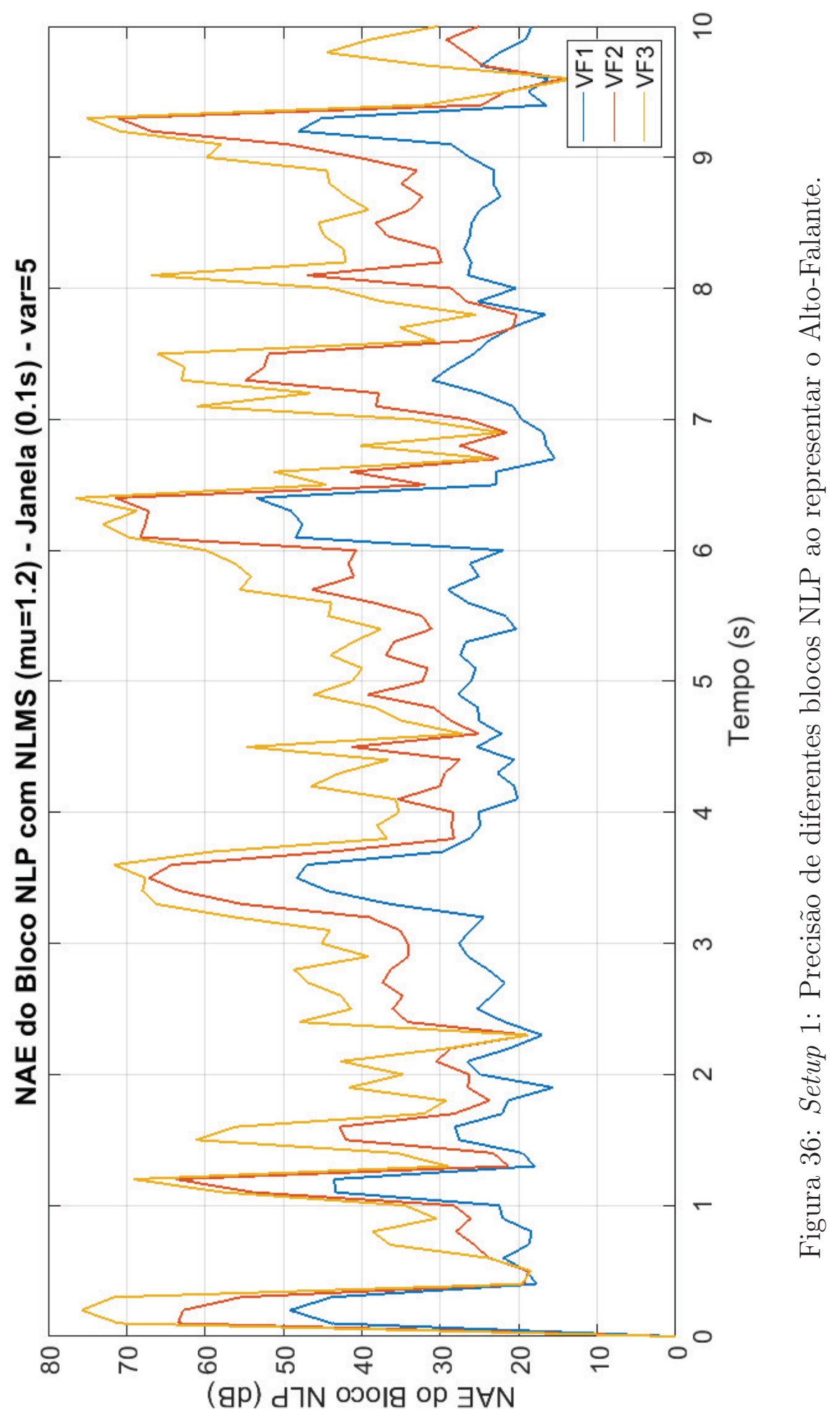


Considerando o Setup 1, o resultado da simulação do NLPAEC(NLML) apresentado na Figura 33 revela de forma geral que as estruturas não-lineares usadas como bloco NLP fizeram com que os valores de ERLE alcançados após aproximadamente $5 \mathrm{~s}$ de simulação se aproximassem do valor limite máximo do cancelador de eco acústico não-linear que é o valor da relação sinal-ruído considerado no processo adaptativo, no caso 40dB.

Pelas Figuras 33, 34 e 35, verifica-se que o Filtro de Volterra de $1^{\mathrm{a}}$ ordem apresenta péssimo desempenho de ERLE, o que já era esperado pois se trata de um modelo linear. O objetivo da inclusão de um modelo linear nessa análise é expor o pior caso de desempenho do cancelador de eco proposto, o qual seria fazer com que o mesmo tenha um comportamento puramente linear.

Dentre os modelos não-lineares utilizados nessa simulação, o modelo de espaço de estados resolvido por ODE15s empregado como bloco NLP representa a melhor aproximação possível do modelo do alto-falante, resultando em um excelente desempenho do cancelador do sistema cancelador de eco proposto, alcançando aproximadamente $35 \mathrm{~dB}$ de ERLE de acordo com a Figura 34.

Os Filtros de Volterra de $2^{\mathrm{a}}$ e $3^{\mathrm{a}}$ ordens apresentam desempenhos muito próximos ao modelo $O D E 15 s$, não sendo possível diferenciar esses modelos em termos de valores de ERLE pela Figura 34.

A Figura 35 revela o desempenho do sistema proposto usando um processo de adaptação sem adição de ruído e a Figura 36 apresenta os valores de NAE do bloco NLP, indicando a precisão do mesmo ao representar o modelo do alto-falante. Por essas figuras, é notavél que as diferenças entre os valores de ERLE dos Filtros de Volterra de $2^{\mathrm{a}}$ e $3^{\mathrm{a}}$ ordens são bem menores do que as diferenças entre seus valores de NAE, indicando o efeito de compensação do filtro adaptativo.

Conclui-se dessa simulação que os modelos Filtro de Volterra de $2^{\mathrm{a}}$ e $3^{\mathrm{a}}$ or- 
dens implementados como bloco NLP aproximam o comportamento do modelo do alto-falante com precisão, como observado pelo excelente desempenho do NLPAEC(NLML) que emprega esses modelos. 


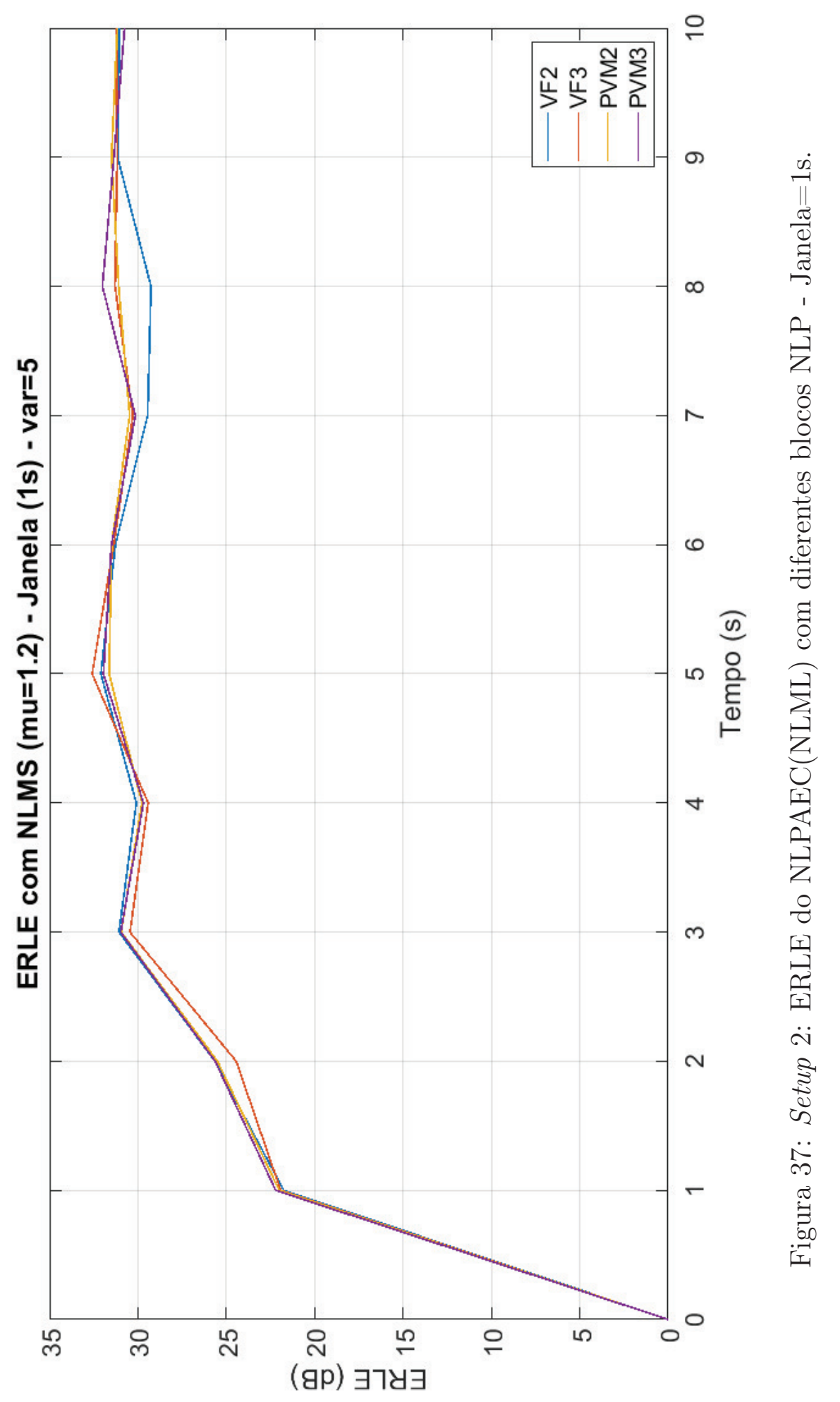




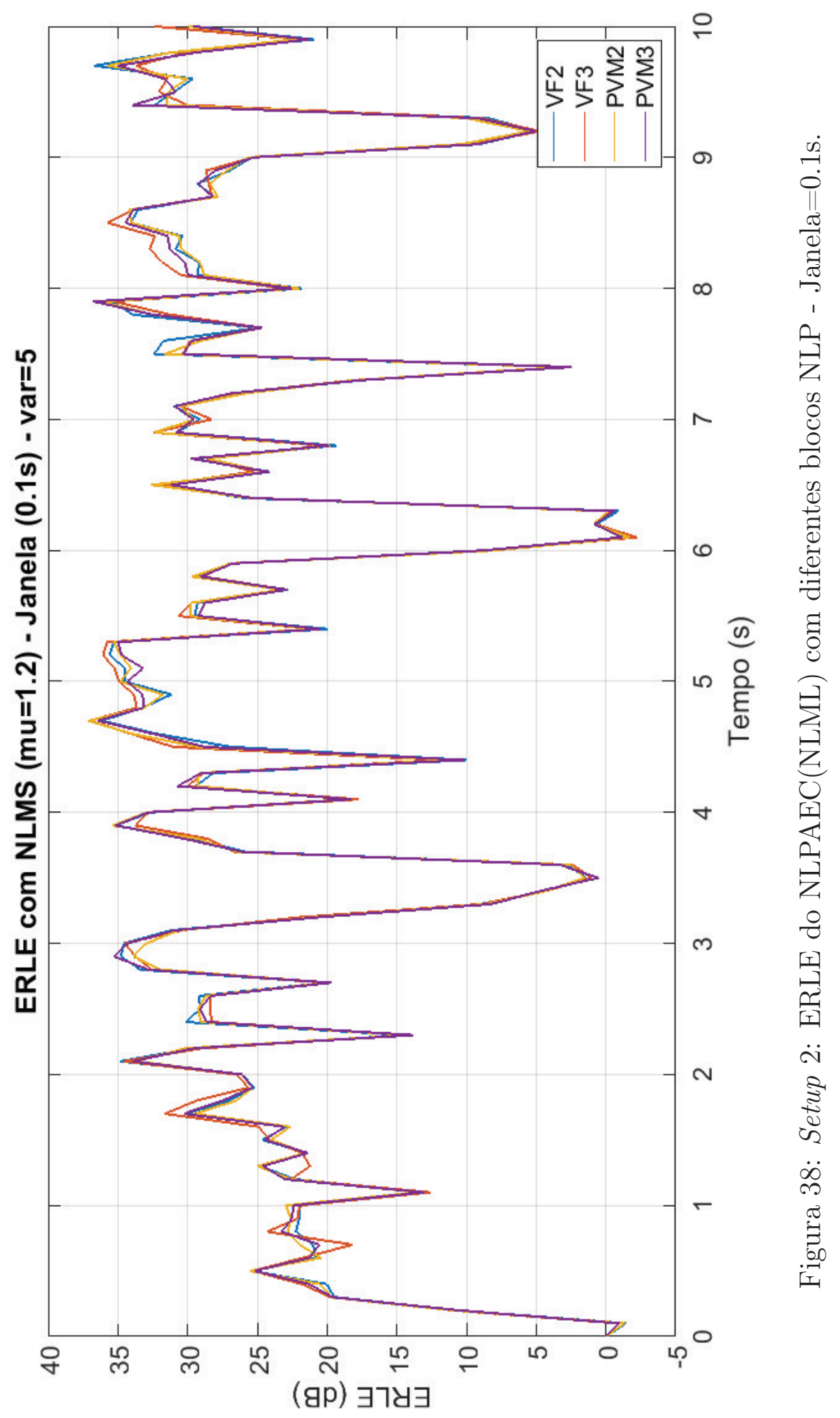




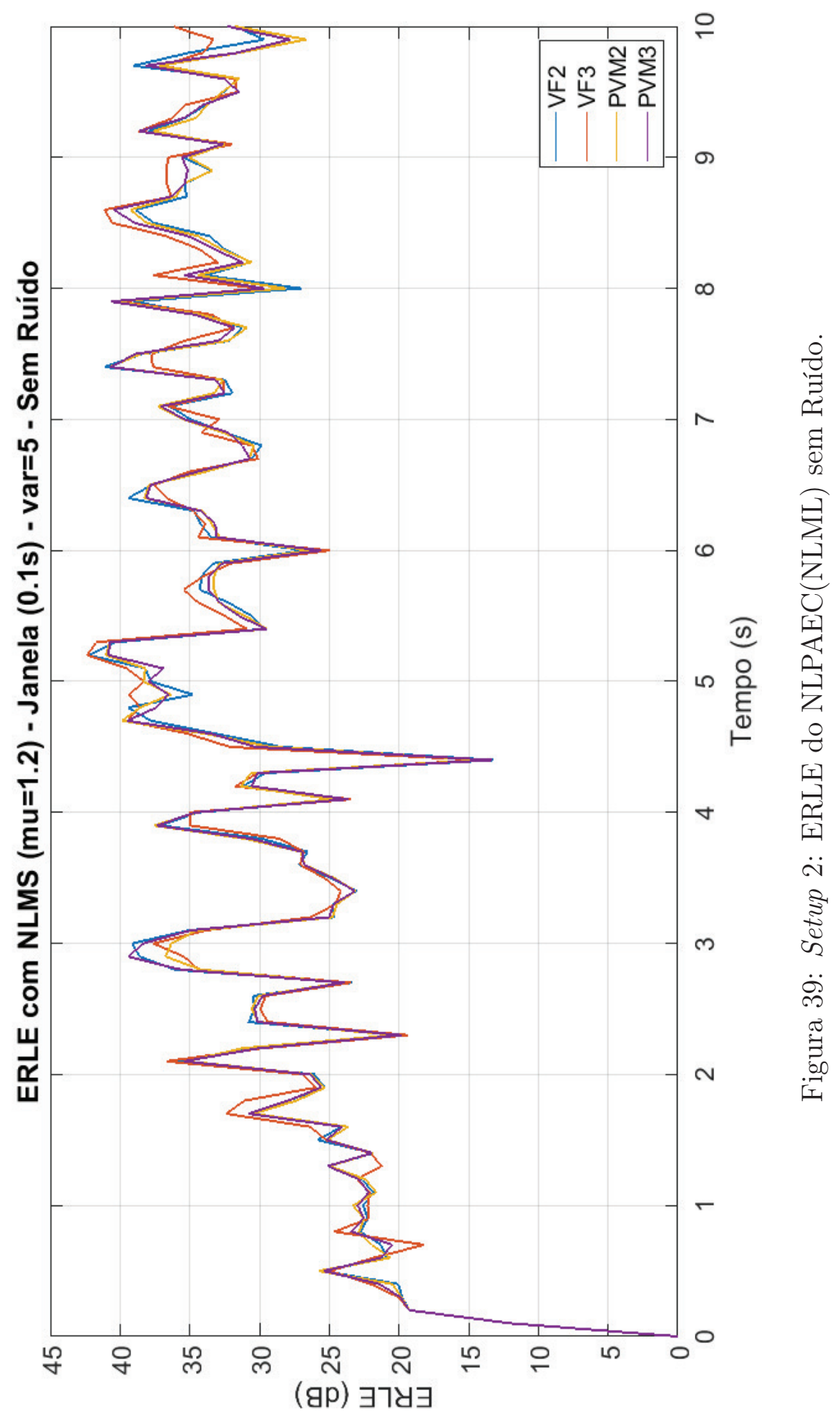




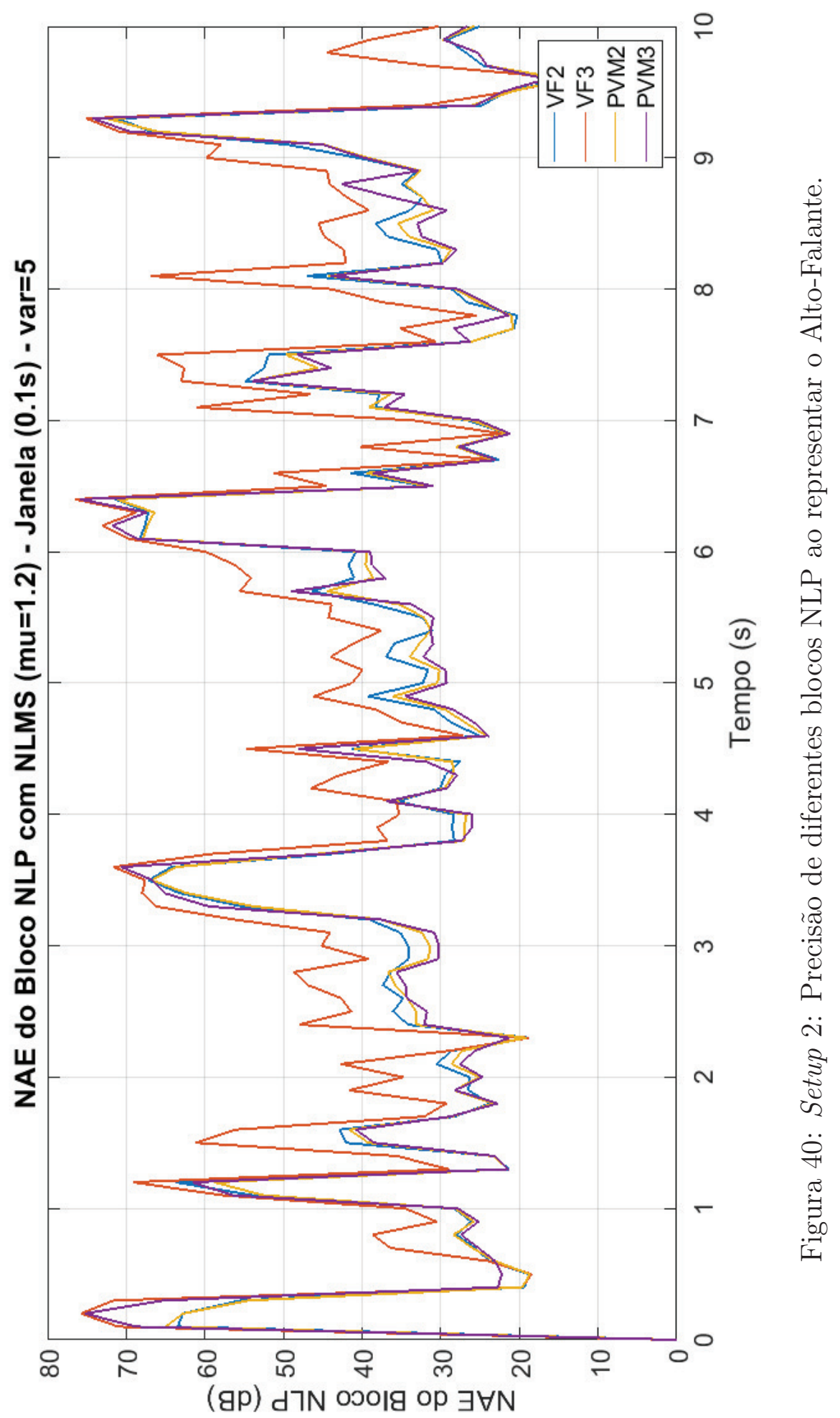


Considerando o Setup 2, o resultado da simulação do NLPAEC(NLML) apresentado na Figura 37 mostra que os modelos de PARAFAC-Volterra de $2^{\mathrm{a}}$ e $3^{\mathrm{a}}$ ordens apresentam comportamento de ERLE muito próximo aos Filtros de Volterra Convencionais de $2^{\mathrm{a}}$ e $3^{\mathrm{a}}$ ordens. Não é possível diferenciar o comportamento desses modelos em termos de valores de ERLE, nem mesmo analisando a Fig. 38 que mostra em mais detalhes o comportamento do sistema proposto.

Pelas Figuras 39 e 40, percebe-se que as diferenças entre os valores de ERLE dos modelos PARAFAC-Volterra e o Filtro de Volterra de $3^{\text {a }}$ ordem são bem menores do que as diferenças entre seus valores de NAE, indicando o efeito de compensação do filtro adaptativo.

Como já mencionado anteriormente no presente trabalho, os modelos PARAFAC-Volterra apresentam complexidade estrutural e carga computacional inferiores aos Filtros de Volterra Convencionais e analisando o comportamento do ERLE do cancelador de eco proposto, NLPAEC(NLML), que utiliza tais estruturas como bloco NLP, conclui-se dessa simulação que os modelos PARAFACVolterra de $2^{\mathrm{a}}$ e $3^{\mathrm{a}}$ ordens apresentam melhor compromisso entre desempenho e carga computacional que os Filtros de Volterra Convencionais. 


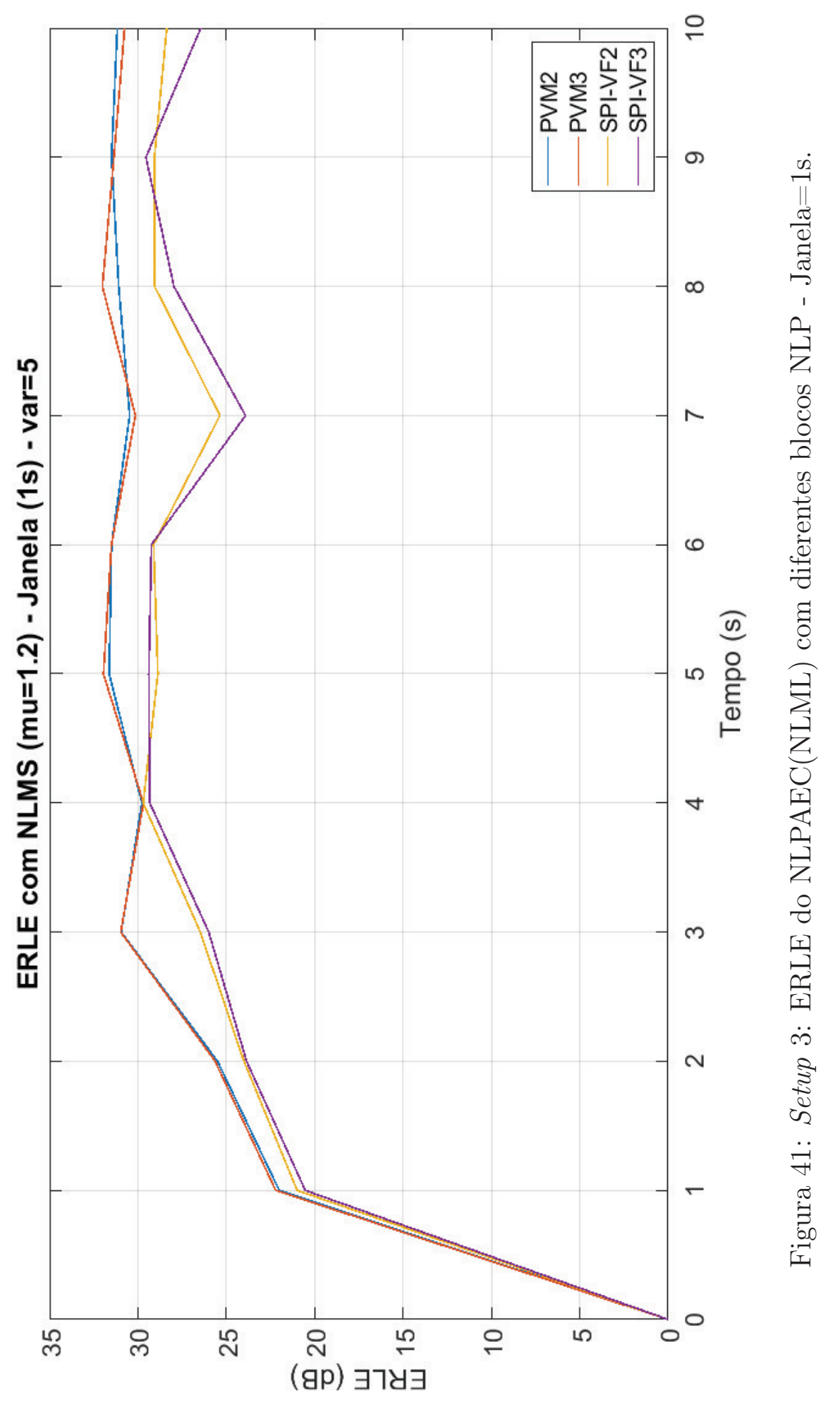




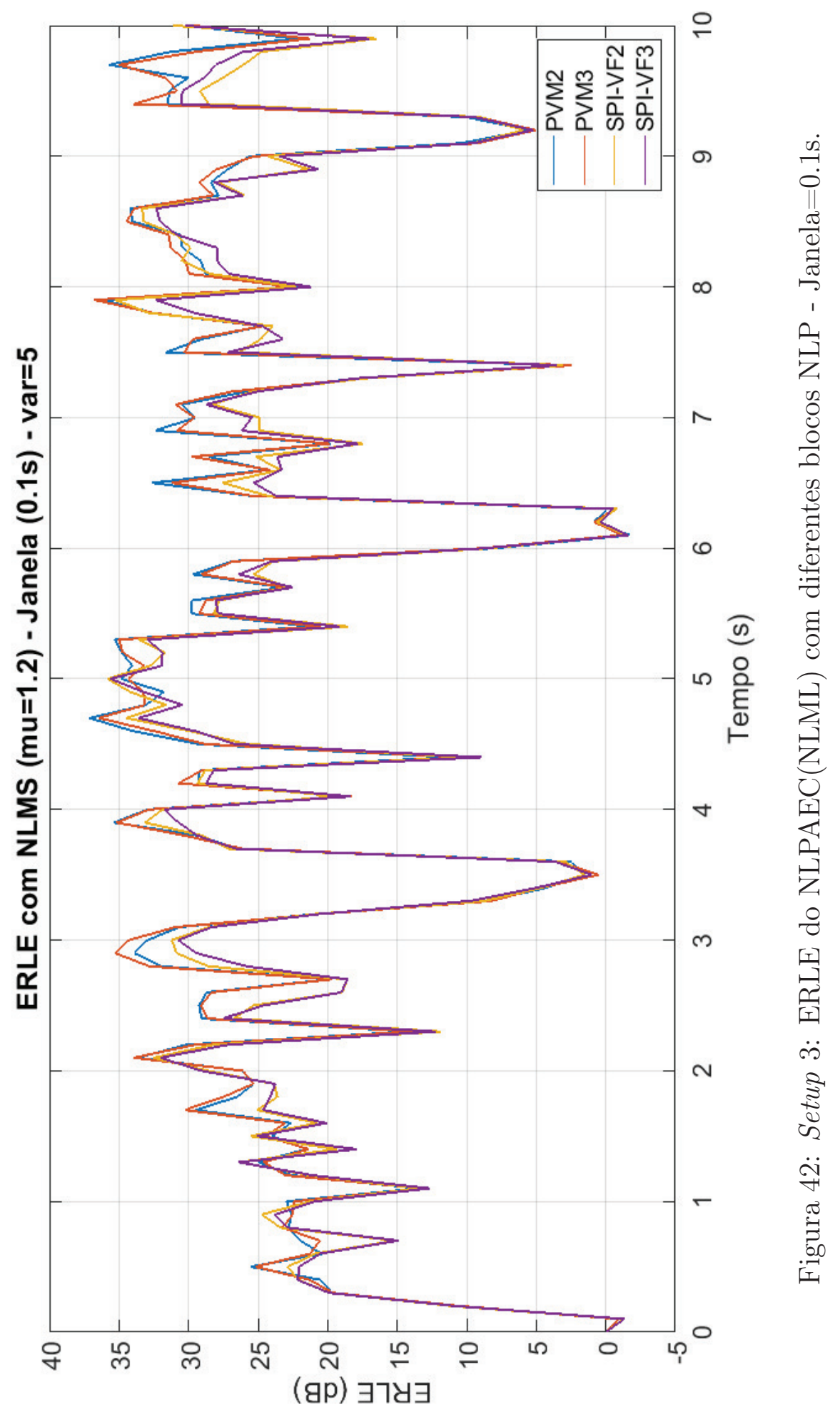




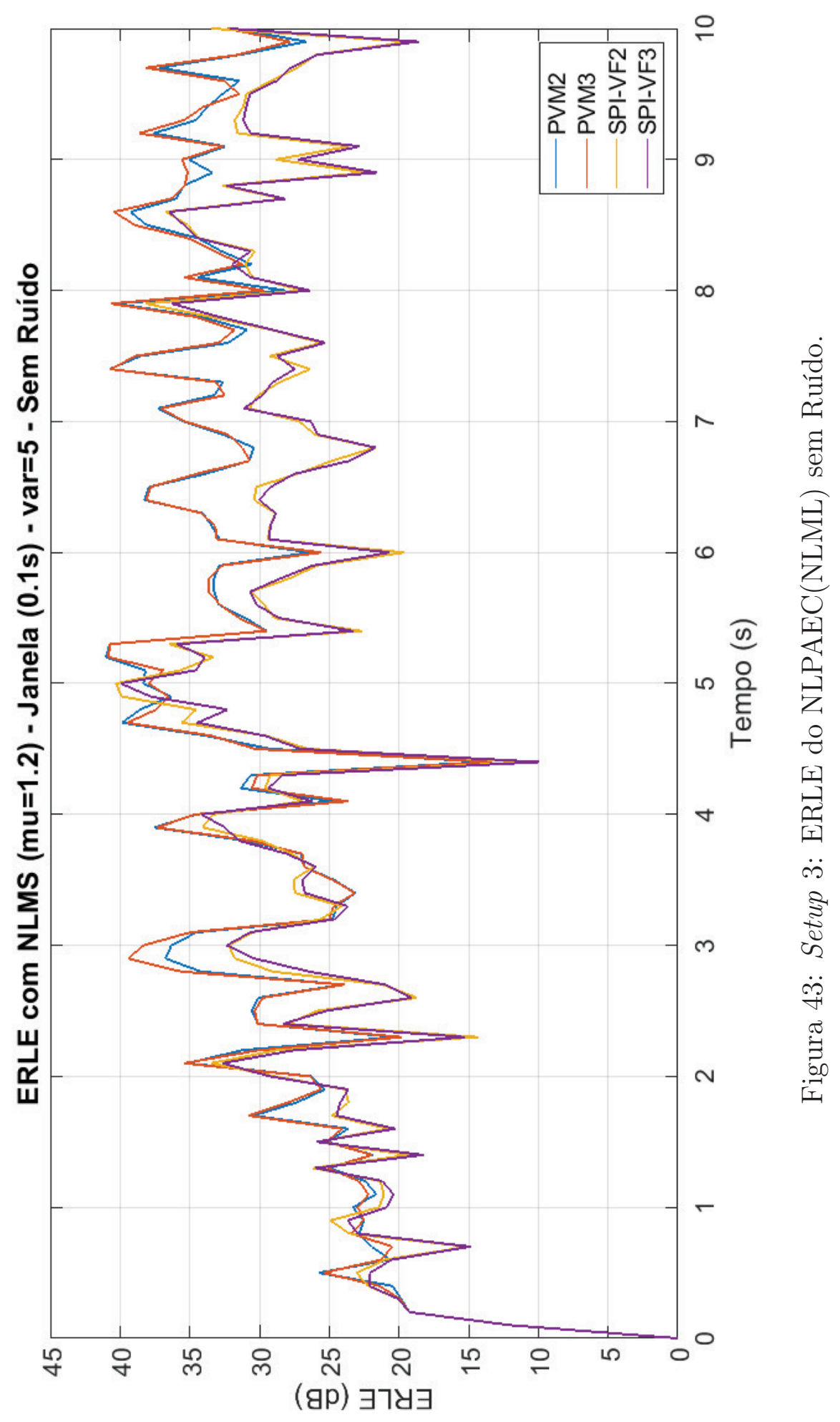




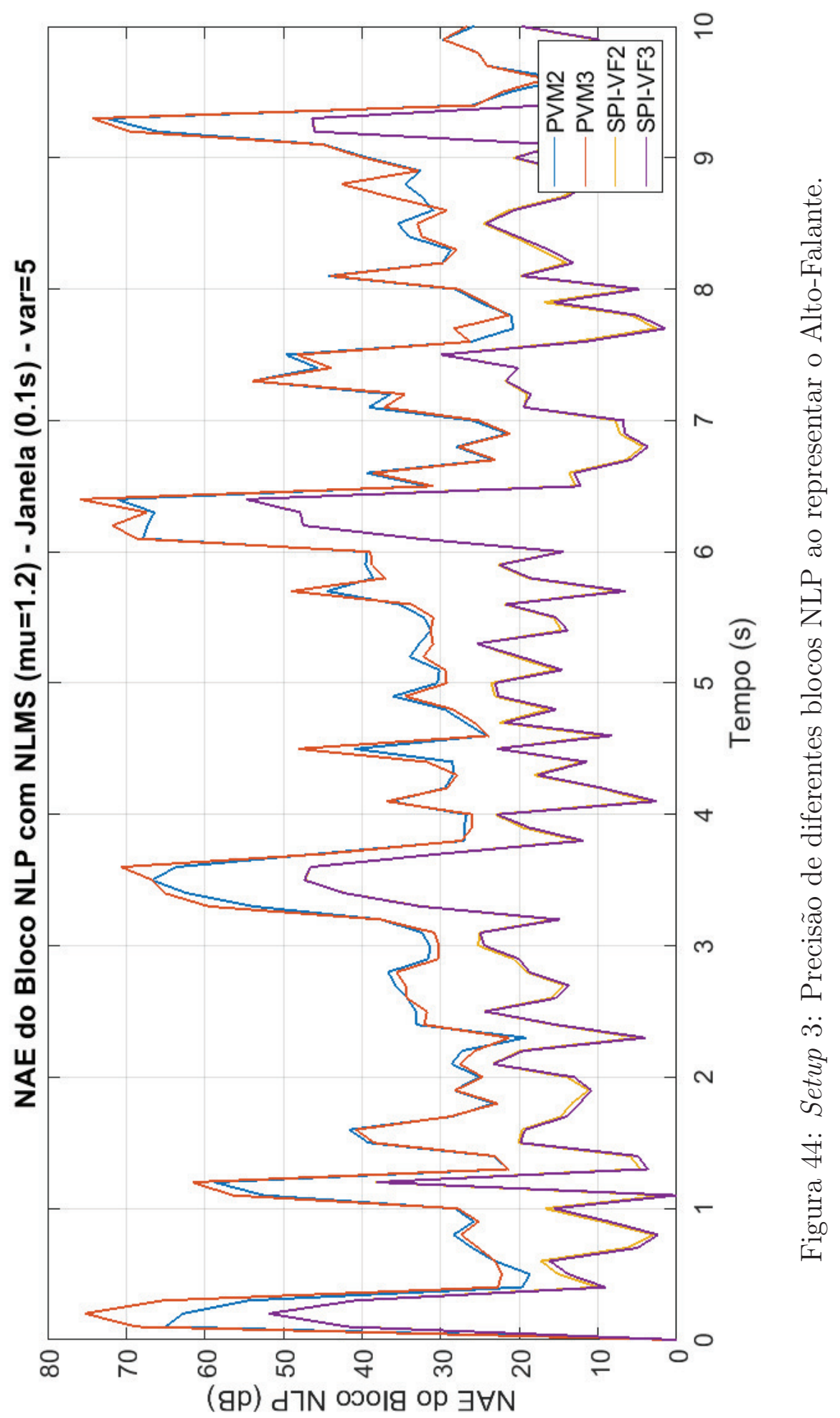


Considerando o Setup 3, os resultados da simulação do NLPAEC(NLML) apresentados nas Figuras 41 e 42 mostram que os Filtros de Volterra Esparso Interpolado apresentam comportamento de ERLE próximo àqueles apresentado pelos modelos PARAFAC-Volterra, porém nota-se um pequeno decaimento de aproximadamente $2 \mathrm{~dB}$ no valor do ERLE alcançado pelos Filtros de Volterra Esparso Interpolado.

Pelas Figuras 43 e 44, percebe-se que as diferenças entre os valores de ERLE dos Filtros de Volterra Esparso Interpolado e os modelos PARAFAC-Volterra são bem menores do que as diferenças entre seus valores de NAE, indicando o efeito de compensação do filtro adaptativo.

Conclui-se dessa simulação que os modelos Filtro de Volterra de $2^{\mathrm{a}}$ e $3^{\mathrm{a}}$ ordens implementados como bloco NLP aproximam o comportamento do modelo do alto-falante com precisão, como observado pelo excelente desempenho do NLPAEC(NLML) que emprega esses modelos.

Baseado no comportamento de ERLE apresentado pelos Filtros de Volterra Esparso Interpolado nessa simulação, conclui-se que essas estruturas não-lineares aproximam o comportamento do modelo do alto-falante com precisão. Fica pendente a análise comparativa mais detalhada da carga computacional dos modelos, a qual será feita na próxima seção. 


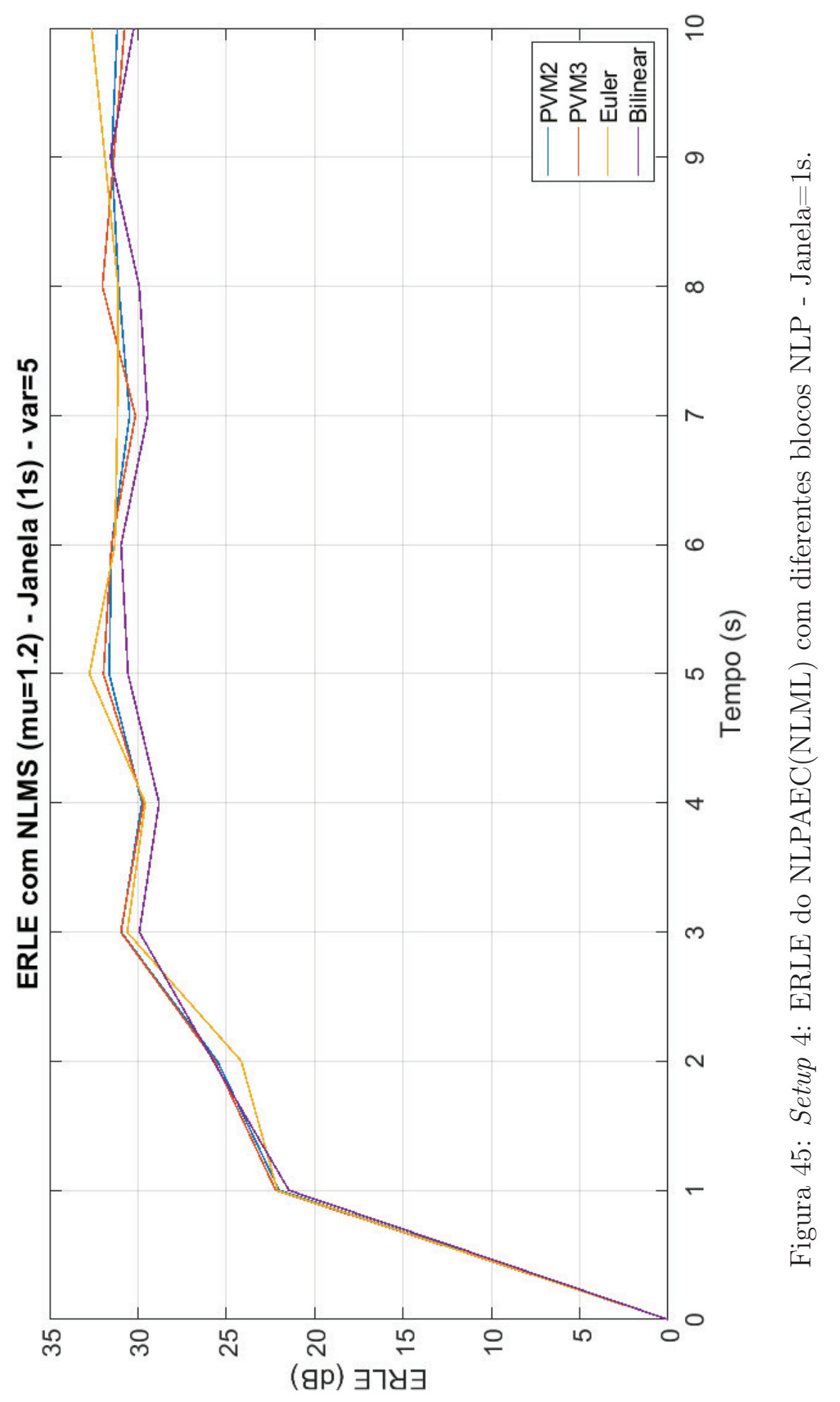




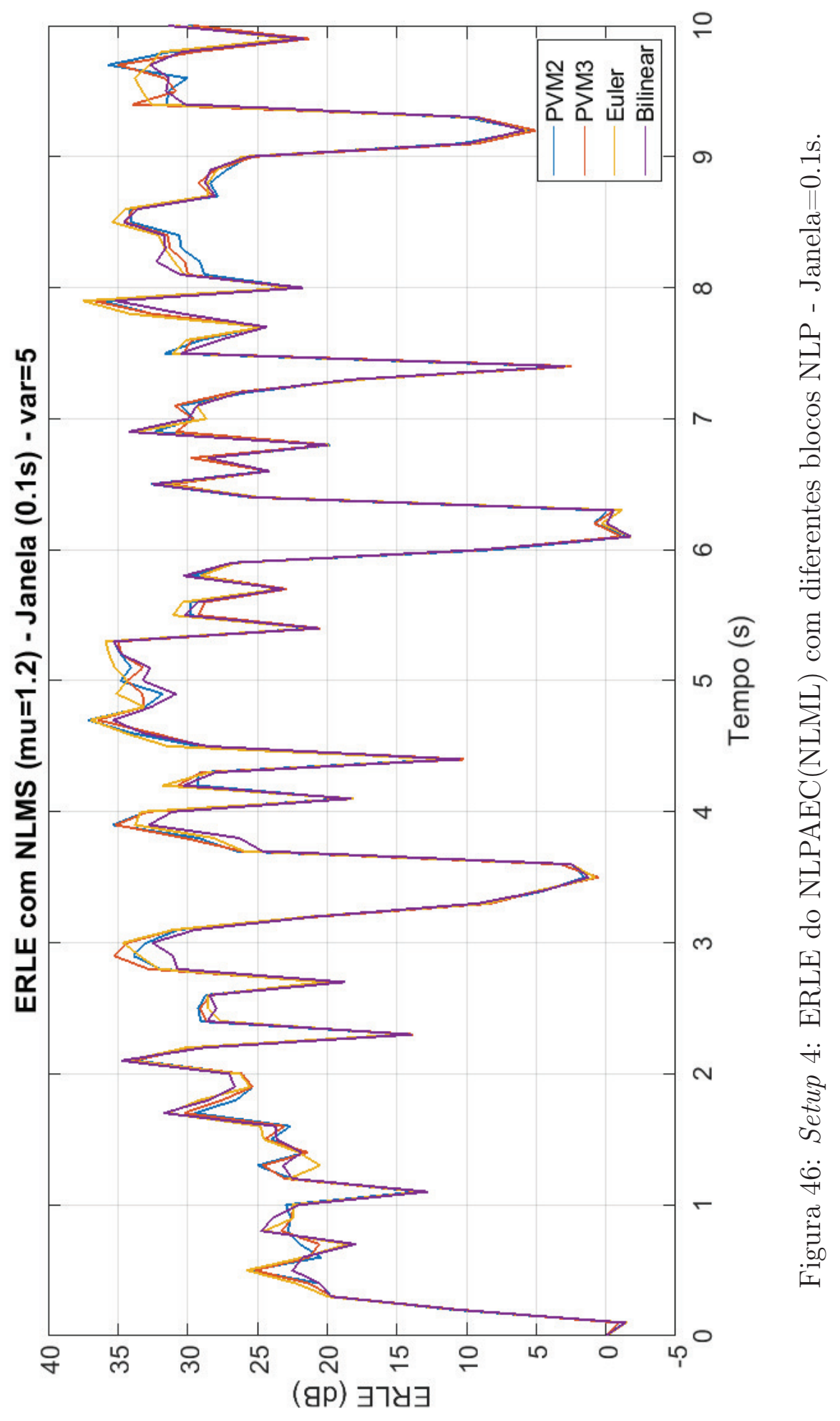




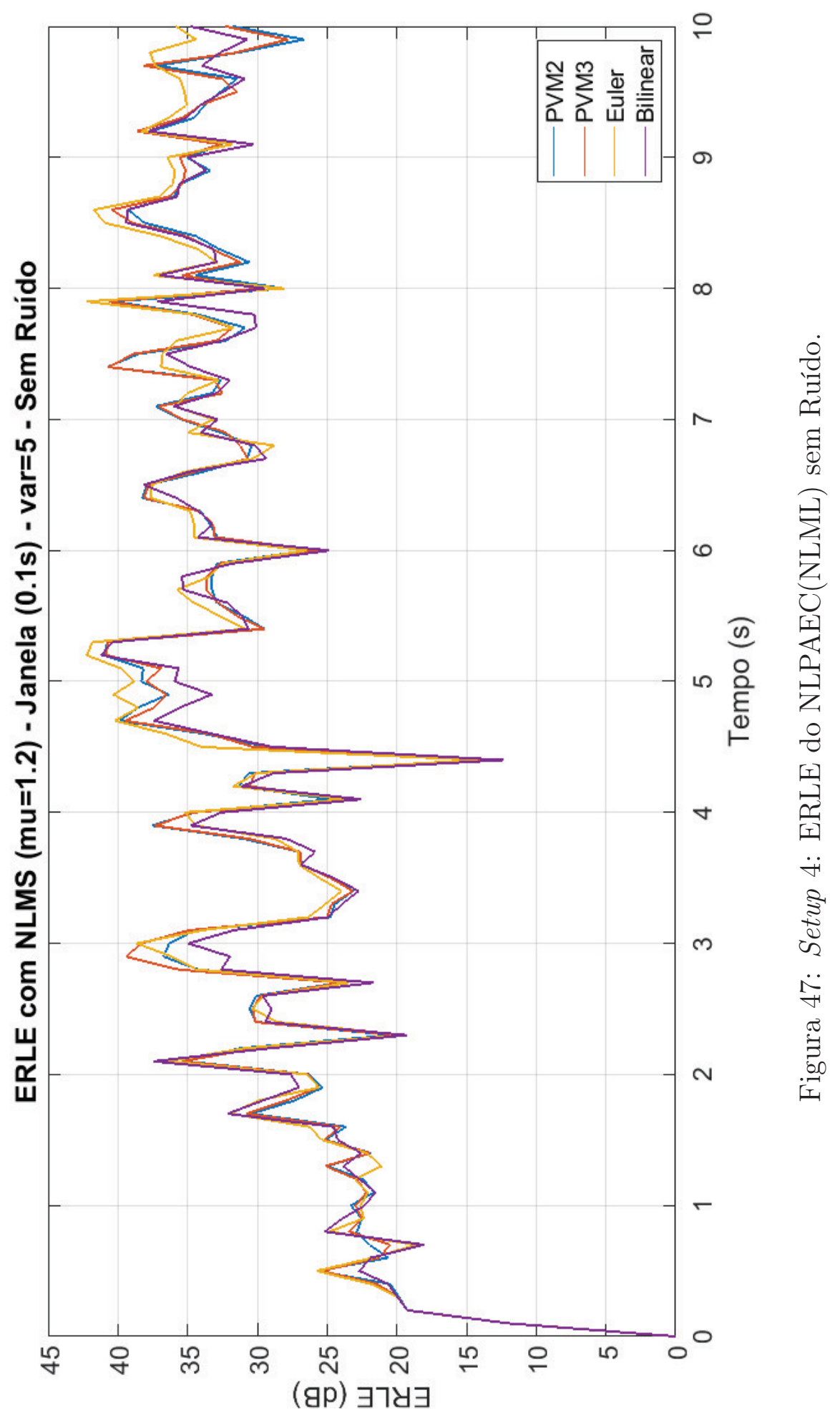




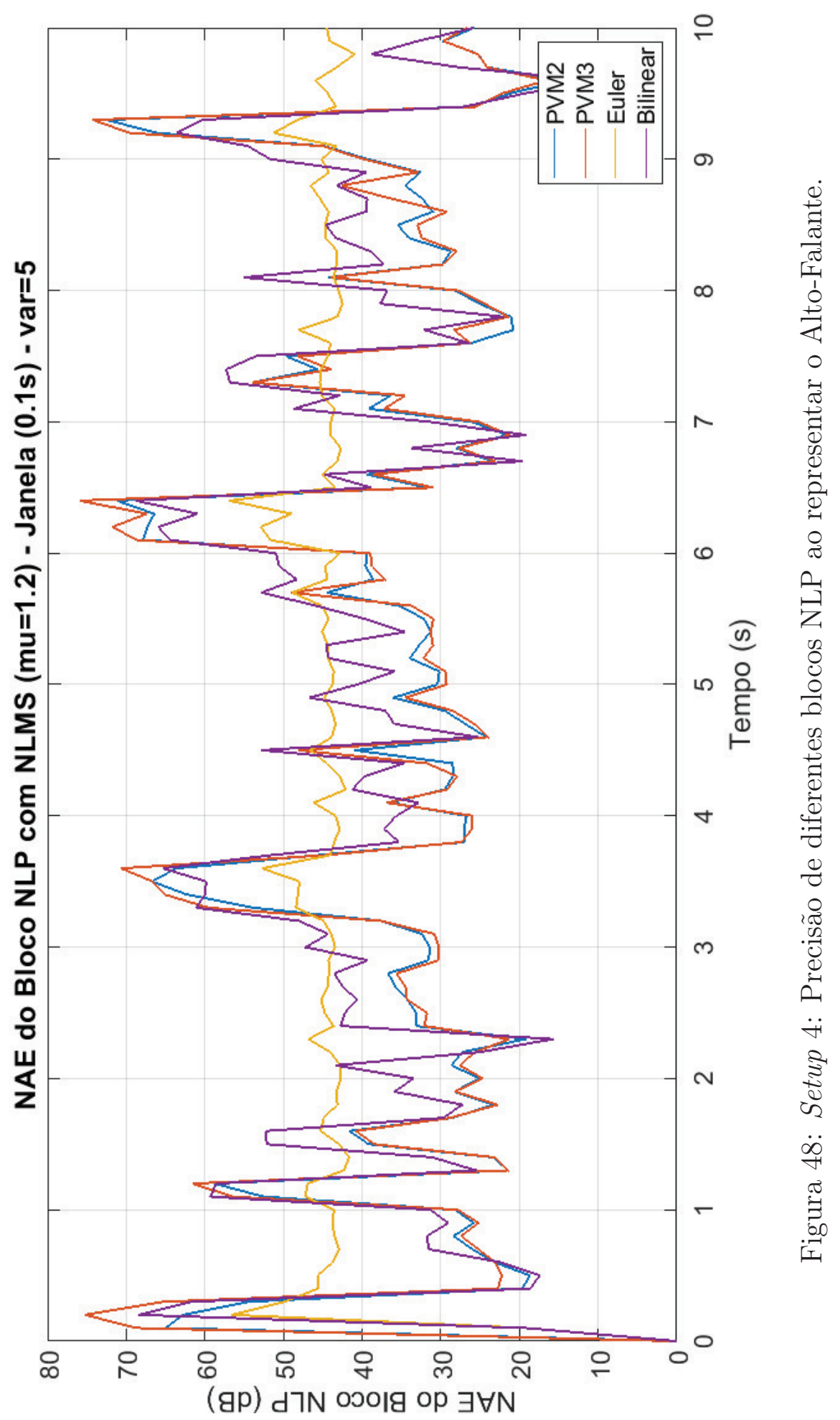


Considerando o Setup 4, os resultados da simulação do NLPAEC(NLML) apresentados nas Figuras 45 e 46 mostram que os modelos baseados na discretização de Euler apresentam desempenho excelente em termos de ERLE. As discretizações pelo método de Euler das equações de espaço de estado e do sistema bilinear, empregados como bloco NLP, permitem ao cancelador de eco acústico proposto alcançar 35db de ERLE, assim como os modelos PARAFAC-Volterra.

O efeito da adaptação, indicados nas Figuras 47 e 48, manifesta-se como compensação da imprecisão dos modelos PARAFAC-Volterra na representação do modelo do alto-falante. Nota-se que as diferenças entre os valores de ERLE dos modelos PARAFAC-Volterra e os modelos de Euler e Bilinear são bem menores do que as diferenças entre seus valores de NAE.

Pelo excelente desempenho em termos de ERLE e altos valores de NAE apresentados nessa simulação pelos modelos de Euler e Bilinear, conclui-se que esses modelos possuem maior precisão na representação do modelo do alto-falante que os modelos não-lineares já apresentados no presente trabalho. Em particular, é interessante notar que devido ao processo de interpolação necessário para simular esses sistemas, as frequências de $220 \mathrm{kHz}$ e $800 \mathrm{KHz}$ foram utilizadas para os modelos de Euler e Bilinear, respectivamente, sendo que abaixo desses valores os sistemas divergiram. Na próxima seção será realizada a análise da carga computacional dos modelos não-lineares empregados como bloco NLP no cancelador de eco acústico proposto. 


\subsubsection{Carga Computacional dos Modelos}

O capítulo 3 apresentou considerações sobre a complexidade estrutural e a carga computacional de cada estrutura não-linear utilizada como bloco NLP do sistema proposto. Nessa seção as expressões para cálculo do número de coeficientes e operações são organizadas em tabelas com o objetivo de consolidar as informações necessárias para a comparação dos modelos em termos de carga computacional necessária para sua implementação.

A Tabela 7 apresenta as expressões para o cálculo do número de coeficientes e operações por amostra do sinal de entrada para os modelos: Volterra Convencional (VF, do inglês Volterra Filter), PARAFAC-Volterra (PVM, do inglês PARAFAC Volterra Model) e Volterra Esparso Interpolado (SPI-VF, do inglês Sparse Interpolated Volterra Filter).

\begin{tabular}{|c|c|c|}
\hline \multicolumn{3}{|c|}{ Complexidade Estrutural e Carga Computacional } \\
\hline Modelo & Ncoef & Nopers \\
\hline VF & $\sum_{p=1}^{P} \frac{\left(N_{p}+p-1\right) !}{p !\left(N_{p}-1\right) !}$ & $\sum_{p=1}^{P}\left(\frac{\left(N_{p}+p-1\right) !}{p !\left(N_{p}-1\right) !}\right) p$ \\
PVM & $N_{1}+\sum_{p=2}^{P} R_{p} N_{p}$ & $N_{1}+\sum_{p=2}^{P} R_{p}\left(N_{p}+p-1\right)$ \\
SPI-VF & $\sum_{p=1}^{P} \frac{\left(N_{p}^{(s)}+p-1\right) !}{p !\left(N_{p}^{(s)}-1\right) !}$ & $\sum_{p=1}^{P}\left(\frac{\left(N_{p}^{(s)}+p-1\right) !}{p !\left(N_{p}^{(s)}-1\right) !}\right) p+(2 L-1)$ \\
\hline
\end{tabular}

Tabela 7: Equações de Complexidade Estrutural e Carga Computacional.

Sendo que as Tabelas 5 e 6 do Capítulo 3 já apresentaram antecipadamente as expressões para o cálculo do número de operações por amostra do sinal de entrada para os modelos: Modelo de Euler e Sistema Bilinear.

Finalmente, após a análise de desempenho dos modelos ter sido concluída na seção anterior, apresentam-se os dados relativos à carga computacional de cada um dos modelos. Esses dados foram calculados conforme mencionado nas Tabelas 5,6 e 7; e levam em consideração as configurações dos modelos utilizadas nas simulações. A Tabela 8 apresenta a carga computacional dos modelos.

A Tabela 8 revela o número de coefientes do modelo (Ncoef), o número de 


\begin{tabular}{|c|c|c|c|c|c|}
\hline \multicolumn{6}{|c|}{ Complexidade Estrutural e Carga Computacional } \\
\hline Modelo & Ncoef & Nopers & Fs & Ntotal & Comparação \\
\hline VF1 & 100 & 100 & 5.000 & 500.000 & $0,98 \%$ \\
VF2 & 5.150 & 10.200 & 5.000 & 51.000 .000 & $100,00 \%$ \\
VF3 & 176.850 & 525.300 & 5.000 & 2.626 .500 .000 & $5.150,00 \%$ \\
PVM2-R3 & 400 & 403 & 5.000 & 2.015 .000 & $3,95 \%$ \\
PVM3-R3 & 700 & 709 & 5.000 & 3.545 .000 & $6,95 \%$ \\
SPI-VF2 & 350 & 689 & 5.000 & 3.445 .000 & $6,75 \%$ \\
SPI-VF3 & 3.275 & 9.471 & 5.000 & 47.355 .000 & $92,85 \%$ \\
Euler & - & 33 & 220.000 & 10.790 .000 & $21,16 \%$ \\
Bilinear & - & 258 & 800.000 & 219.210 .000 & $429,82 \%$ \\
\hline
\end{tabular}

Tabela 8: Análise da carga computacional dos modelos.

operações por amostra (Nopers), a frequência de amostragem (Fs) ou número de amostras processadas em 1s, o número total de operações (Ntotal) realizadas em 1s e, finalmente, uma análise comparativa percentual (Comparação) entre os modelos baseada em uma escala percentual de carga computacional.

A análise comparativa percentual entre os modelos revela o quanto uma estrutura não-linear empregada como bloco NLP é mais ou menos complexa computacionalmente em relação ao modelo de referência previamente selecionado. Essa complexidade de implementação é determinada pelo número total de operações (Ntotal) necessárias para que a saída de um determinado modelo seja totalmente computada considerando um sinal de entrada de 1s de duração, ou seja, é determinada pela carga computacional necessária para o cômputo da saída do modelo.

Baseado na literatura, sabe-se que o Filtro de Volterra Convencional é o modelo com maior carga computacional dentre os modelos de Volterra considerados no presente trabalho e que o Filtro de Volterra de $2^{\mathrm{a}}$ ordem possui carga computacional mais próxima dos outros modelos de Volterra que o Filtro de Volterra de $3^{\mathrm{a}}$ ordem. Tal fato determina a escolha do Filtro de Volterra de $2^{\mathrm{a}}$ ordem como a referência para a comparação entre os modelos, indicando que as 51.000 .000 operações necessárias para o processamento do sinal de entrada em 1s correspondem a $100 \%$ de carga computacional, como mostra a Tabela 8 . 
Nessa seção adota-se como critério de classificação da carga computacional de cada estrutura não-linear os seguintes valores e rótulos: $<5 \%$ - Excelente; $<$ $10 \%$ - Ótima; < 15\% - Boa; <50\% - Regular. Destaca-se o fato de que qualquer valor percentual de carga computacional abaixo de $100 \%$ pode ser considerada como relativamente baixa, porém nessa seção procura-se identificar os modelos não lineares que possuem classificação pelo menos Boa.

O Filtro de Volterra Convencional de $3^{\text {a }}$ ordem tem carga computacional 51 vezes maior que o Filtro de Volterra Convencional de $2^{\mathrm{a}}$ ordem, apresentando valor de 5.150,00\% na coluna de comparação na Tabela 8. Portanto, o Filtro de Volterra Convencional de $3^{\mathrm{a}}$ ordem apresenta compromisso entre desempenho e carga computacional ruim para seu uso como bloco NLP no cancelador de eco proposto, pois sua complexidade de implementação é grande, apesar do excelente desempenho em termos de ERLE mencionado na seção anterior.

Observa-se pela tabela que o modelo cuja implementação é menos complexa é o Filtro de Volterra Convencional de $1^{\mathrm{a}}$ ordem, pois necessita de apenas 0,98\% da carga computacional com relação ao Filtro de Volterra Convencional de $2^{\mathrm{a}}$ ordem. Porém, considerando a simulação realizada no Setup 1, descrita na seção anterior, sabe-se que o desempenho do cancelador de eco acústico utilizando esse modelo linear é péssimo, portanto, o Filtro de Volterra Convencional de $1^{\mathrm{a}}$ ordem não é uma opção adequada a ser empregada como bloco NLP, apesar de sua facilidade de implementação.

A mesma conclusão de inadequação ao uso como bloco NLP feita no parágrafo anterior é aplicável à implementação da estrutura não-linear resultante da discretização de Euler do Sistema Bilinear. De acordo com a tabela, 429,82\% de carga computacional é necessária para sua implementação, o que representa uma complexidade de implementação elevada e, apesar da simulação do Setup 4 revelar que o desempenho em termos de ERLE desse modelo é um dos melhores, 
sua implementação é muito complexa.

De acordo com os dados apresentados na tabela e pelo resultado da simulação do Setup 2, os modelos PARAFAC-Volterra de $2^{\mathrm{a}}$ e $3^{\mathrm{a}}$ ordens representam excelentes opções para utilização como bloco NLP, pois as cargas computacionais são extremamente baixas, 3,95\% e 6,95\%, respectivamente. Além disso, ambas estruturas não-lineares apresentaram excelente desempenho em termos de ERLE, alcançando $35 \mathrm{db}$.

Os modelos SPI-VF de $2^{\mathrm{a}}$ e $3^{\mathrm{a}}$ ordens também apresentam carga computacional relativamente baixa, como nota-se pela Tabela 8. Porém, esses modelos não apresentaram bom desempenho com relação aos valores de ERLE, alcançando apenas $33 \mathrm{~dB}$, como descrito nos resultados da simulação do Setup 3 da seção anterior. Analisando a tabela, descarta-se a utilização do modelo SPI-VF de $3^{\text {a }}$ ordem como bloco NLP, pois esse modelo apresenta carga computacional de $92,85 \%$, a qual é muito superior ao valor obtido para o SPI-VF de $2^{\text {a }}$ ordem que é de 6,75\%. Contudo, avalia-se a possibilidade de utilização do modelo SPI-VF de $2^{\mathrm{a}}$ ordem como bloco NLP no próximo capítulo, pois esse modelo apresenta bom compromisso entre desempenho e carga computacional.

Por último, analisa-se a carga computacional da estrutura não-linear resultante da discretização pelo método de Euler das equações de espaço de estado do modelo físico do alto-falante. Baseando-se na tabela, observa-se que o modelo apresenta complexidade de implementação regular, 21,16\% da complexidade do VF2. Apesar da simulação do Setup 4 da seção anterior mostrar que o modelo de Euler apresenta excelente desempenho em termos de ERLE, esse modelo não representa uma boa opção para implementação do bloco NLP, pois não apresenta um bom compromisso entre desempenho e carga computacional. 


\section{CONCLUSÕES}

O presente trabalho teve por objetivo analisar o compromisso entre desempenho e carga computacional das estruturas não-lineares a serem utilizadas como bloco NLP no cancelador de eco proposto, NLPAEC(NLML), e determinar qual a melhor opção para implementação do mesmo.

Com base nos resultados das simulações, dados de carga computacional e análise realizada no capítulo anterior, conclui-se que existem 3 estruturas nãolineares que apresentaram compromisso entre desempenho e carga computacional satisfatório com base nos critérios estabelecidos nas seções 5.3.1 e 5.3.2. Cada uma dessas estruturas é listada abaixo, destacando-se os valores de carga computacional previamente calculados e de ERLE alcançados nas simulações.

\begin{tabular}{|c|c|c|c|c|}
\hline \multicolumn{5}{|c|}{ Compromisso entre Desempenho x Carga Computacional } \\
\hline Modelo & Ntotal & Comparação & ERLE & Compromisso \\
\hline PVM2-R3 & 2.015 .000 & $3,95 \%$ & $35 \mathrm{~dB}$ & Excelente \\
PVM3-R3 & 3.545 .000 & $6,95 \%$ & $35 \mathrm{~dB}$ & Ótimo \\
SPI-VF2 & 3.445 .000 & $6,75 \%$ & $33 \mathrm{~dB}$ & Bom \\
\hline
\end{tabular}

Tabela 9: Carga computacional e valores de ERLE dos modelos.

Observa-se pela tabela que o único modelo que apresentou desempenho em termos de ERLE abaixo de $35 \mathrm{~dB}$ foi o Filtro de Volterra Esparso Interpolado de $2^{\mathrm{a}}$ ordem (SPI-VF2), alcançando 33dB, enquanto os demais modelos apresentaram 35dB de ERLE. Assim, classifica-se o compromisso entre desempenho e carga computacional desse modelo como Bom. Apesar disso, vale destacar que em situações em que o ruído é extremamente baixo ou nulo, como descrito na Figura 
43 da seção 5.3.1, essa estrutura não-linear resulta em um sistema proposto com desempenho muito inferior se comparado ao uso do modelo PARAFAC-Volterra como bloco NLP.

O cancelador de eco acústico proposto empregando o modelo PARAFACVolterra como bloco NLP apresentou desempenho excelente em termos de ERLE e a carga computacional associada a esse modelo é muito baixa, conforme pode ser constatado pela análise da Tabela 9. Porém, verifica-se que a carga computacional do modelo PVM3 é aproximadamente 2 vezes maior que a do modelo PVM2, 6,95\% e 3,95\% respectivamente. Logo, o modelo PARAFAC-Volterra de $3^{\text {a }}$ ordem apresenta ótimo compromisso entre desempenho e carga computacional, ficando atrás apenas do modelo PARAFAC-Volterra de $2^{\mathrm{a}}$ ordem.

Convertendo os percentuais relativos aos modelos PARAFAC-Volterra em números de operações totais, conforme apresentados na tabela, nota-se que são necessárias mais de 1,5 milhões de operações adicionais para implementação do modelo PVM3 em comparação ao modelo PVM2, o qual necessita apenas de aproximadamente 2 milhões de operações para sua implementação como bloco NLP no sistema proposto. Em particular, o modelo PARAFAC-Volterra de $2^{\text {a }}$ ordem apresenta excelente compromisso entre desempenho e carga computacional.

Importante ressaltar o fato de que o filtro adaptativo, mesmo que puramente linear, compensa pequenas imperfeições do modelo do alto-falante não-linear, tendo um papel fundamental no desempenho alcançado pelo cancelador de eco acústico não-linear proposto.

Após análise criteriosa dos resultados de simulações que revelaram o desempenho dos modelos e avaliação dos dados das cargas computacionais envolvidas para implementação dos mesmos, determina-se que o modelo PARAFAC-Volterra de $2^{\text {a }}$ ordem é a melhor opção para implementação do bloco NLP, pois apresenta o melhor compromisso dentre todos os modelos considerados, necessitando de carga 
computacional mínima, a qual não compromete o desempenho do cancelador de eco proposto. 


\section{PERSPECTIVAS DE TRABALHOS FUTUROS}

Alguns aspectos observados no presente trabalho precisam ainda ser investigados em maiores detalhes, tais como:

1. Estudo da influência da correlação do sinal de entrada no desempenho do cancelador de eco acústico não-linear.

2. Melhor entendimento da compensação, verificada na prática, da imprecisão do bloco não-linear pelo filtro adaptativo linear.

Além disso, algumas situações ainda mais realistas com relação ao ambiente reverberante poderiam ser exploradas. Dentre elas pode-se citar:

1. Consideração do NLPAEC na presença de um sinal de locutor local não nulo, ou seja, o aspecto denominado de double talk na literatura.

2. Avaliação do desempenho do sistema proposto em um contexto nãoestacionário. 


\section{REFERÊNCIAS}

[1] C. Breining, P. Dreiscitel, E. Hansler, A. Mader, B. Nitsch, H. Puder, T. Schertler, G. Schmidt, and J. Tilp. Acoustic echo control. an application of very-high-order adaptive filters. Signal Processing Magazine, IEEE, 16(4):42-69, Jul 1999.

[2] A.N. Birkett and R.A. Goubran. Limitations of handsfree acoustic echo cancellers due to nonlinear loudspeaker distortion and enclosure vibration effects. In Applications of Signal Processing to Audio and Acoustics, 1995., IEEE ASSP Workshop on, pages 103-106, Oct 1995.

[3] A. Stenger, L. Trautmann, and R. Rabenstein. Nonlinear acoustic echo cancellation with 2 nd order adaptive volterra filters. In Acoustics, Speech, and Signal Processing, 1999. Proceedings., 1999 IEEE International Conference on, volume 2, pages 877-880 vol.2, Mar 1999.

[4] M.I. Mossi, N.W.D. Evans, and C. Beaugeant. An assessment of linear adaptive filter performance with nonlinear distortions. In Acoustics Speech and Signal Processing (ICASSP), 2010 IEEE International Conference on, pages 313-316, March 2010.

[5] M.I. Mossi, C. Yemdji, N. Evans, C. Beaugeant, and P. Degry. Robust and low-cost cascaded non-linear acoustic echo cancellation. In Acoustics, Speech and Signal Processing (ICASSP), 2011 IEEE International Conference on, pages 89-92, May 2011.

[6] Riitta Niemistö and Tuomo Mäkelä. On performance of linear adaptive filtering algorithms in acoustic echo control in presence of distorting loudspeakers. In International Workshop on Acoustic Echo and Noise Control (IWAENC2003), Kyoto, Japan, Japan, Kyoto, Sept. 2003.

[7] K. Shi, Xiaoli Ma, and G.T. Zhou. A residual echo suppression technique for systems with nonlinear acoustic echo paths. In Acoustics, Speech and Signal Processing, 2008. ICASSP 2008. IEEE International Conference on, pages 257-260, March 2008.

[8] Fabian Kuech. Approaches to nonlinear acoustic echo cancellation. In Voice Communication (SprachKommunikation), 2008 ITG Conference on, pages $1-4$, Oct 2008.

[9] Alex Stenger, Walter Kellermann, and Rudolf Rabenstein. Adaptation of acoustic echo cancellers: Incorporating a memoryless nonlinearity. In Proc. IEEE Workshop on Acoustic Echo and Noise Control (IWAENC'99), 1999. LMS::307. 
[10] Hans Schurer. Linearization of Electroacoustic Transducers. PhD thesis, Enschede, the Netherlands, November 1997.

[11] J. . Costa, A. Lagrange, and A. Arliaud. Acoustic echo cancellation using nonlinear cascade filters. In 2003 IEEE International Conference on Acoustics, Speech, and Signal Processing, 2003. Proceedings. (ICASSP '03)., volume 5, pages V-389, April 2003.

[12] M. Scarpiniti, D. Comminiello, R. Parisi, and A. Uncini. Comparison of hammerstein and wiener systems for nonlinear acoustic echo cancelers in reverberant environments. In 2011 17th International Conference on Digital Signal Processing (DSP), pages 1-6, July 2011.

[13] F. Kuech, A. Mitnacht, and W. Kellermann. Nonlinear acoustic echo cancellation using adaptive orthogonalized power filters. In Acoustics, Speech, and Signal Processing, 2005. Proceedings. (ICASSP '05). IEEE International Conference on, volume 3, pages iii/105-iii/108 Vol. 3, March 2005.

[14] R. Mortensen. Nonlinear system theory: The volterra/wiener approach (wilson j. rugh). SIAM Review, 25(3):412-414, 1983.

[15] A. Guerin, G. Faucon, and R. Le Bouquin-Jeannes. Nonlinear acoustic echo cancellation based on volterra filters. Speech and Audio Processing, IEEE Transactions on, 11(6):672-683, Nov 2003.

[16] A. Uncini, A. Nalin, and R. Parisi. Acoustic echo cancellation in the presence of distorting loudspeakers. In 2002 11th European Signal Processing Conference, pages 1-4, Sep. 2002.

[17] J. H. d. M. Goulart and P. M. S. Burt. Efficient kernel computation for volterra filter structure evaluation. IEEE Signal Processing Letters, 19(3):135138, March 2012.

[18] J.H.M. Goulart. Derivação eficiente e utilização de filtros de volterra de referência na avaliação de formalismos não-lineares. Dissertação de Mestrado, Escola Politécnica da Universidade de São Paulo, 2012.

[19] D. Franken, K. Meerkotter, and J. Wassmuth. Passive parametric modeling of dynamic loudspeakers. Speech and Audio Processing, IEEE Transactions on, 9(8):885-891, Nov 2001.

[20] E.L.O. Batista, O.J. Tobias, and R. Seara. A sparse-interpolated scheme for implementing adaptive volterra filters. Signal Processing, IEEE Transactions on, 58(4):2022-2035, April 2010.

[21] Gérard Favier, Alain Y. Kibangou, and Thomas Bouilloc. Nonlinear system modeling and identification using Volterra-PARAFAC models. International Journal of Adaptive Control and Signal Processing, 26(1):30-53, January 2012. 
[22] Saeed V. Vaseghi. Multimedia Signal Processing. John Wiley \& Sons, Ltd, 2007.

[23] Man Mohan Sondhi. The history of echo cancellation. Signal Processing Magazine, IEEE, 23(5):95-102, Sept 2006.

[24] M.M. Sondhi. An adaptive echo canceller. Bell System Technical Journal, The, 46(3):497-511, March 1967.

[25] Eberhard Hänsler. The hands-free telephone problem: an annotated bibliography update. Annals of Telecommunications, 49(7-8):360-367, 1994.

[26] G. Glentis, K. Berberidis, and S. Theodoridis. Efficient least squares adaptive algorithms for fir transversal filtering. Signal Processing Magazine, IEEE, 16(4):13-41, Jul 1999.

[27] Kazuhiko Ozeki and Tetsuo Umeda. An adaptive filtering algorithm using an orthogonal projection to an affine subspace and its properties. Electronics and Communications in Japan (Part I: Communications), 67(5):19-27, 1984.

[28] F. Kuch, M. Zeller, and W. Kellermann. Input signal decorrelation applied to adaptive second-order volterra filters in the time domain. In Proc. IEEE 12th Digital Signal Processing Workshop/4th Signal Processing Education Workshop, pages 348-353, September 2006.

[29] Paulo Sergio Diniz. Adaptive Filtering: Algorithms and Practical Implementation. Kluwer Academic Publishers, Norwell, MA, USA, 2 edition, 2002.

[30] Alan V. Oppenheim and Ronald W. Schafer. Discrete-Time Signal Processing. Prentice Hall Press, Upper Saddle River, NJ, USA, 3rd edition, 2009.

[31] Simon Haykin. Adaptive Filter Theory (3rd Ed.). Prentice-Hall, Inc., Upper Saddle River, NJ, USA, 1996.

[32] Levy Boccato, Rafael Ferrari, and Romis Attux. Analysis of the normalized lms optimum solution in the context of channel equalization. Journal of Communication and Information Systems, 2018:22-30, 012018.

[33] J.H.M. Goulart and P.M.S. Burt. Carleman bilinearization and volterra kernels toolbox. [Online] Available:http://www.lcs.poli.usp.br/phillip/sw/cbvk., Dec 2016.

[34] Iain. Loudspeaker cross section. Image by Rohitbd. [Online] Available: http://en.wikipedia.org/wiki/File:Speaker-cross-section.svg., Jan 2007.

[35] Andrzej Dobrucki. Nontypical effects in an electrodynamic loudspeaker with a nonhomogeneous magnetic field in the air gap and nonlinear suspensions. J. Audio Eng. Soc, 42(7/8):565-576, 1994.

[36] Francois E. Cellier and Ernesto Kofman. Continuous System Simulation. Springer-Verlag, Berlin, Heidelberg, 2006. 
[37] S. Rajan, Sichun Wang, R. Inkol, and A. Joyal. Efficient approximations for the arctangent function. IEEE Signal Processing Magazine, 23(3):108-111, May 2006.

[38] Zhaojun Bai and Daniel Skoogh. A projection method for model reduction of bilinear dynamical systems. Linear Algebra and its Applications, 415(2):406 - 425, 2006. Special Issue on Order Reduction of Large-Scale Systems.

[39] David Elliott. Bilinear Control Systems: Matrices in Action. Springer Publishing Company, Incorporated, 1st edition, 2009.

[40] V.J. Mathews. Adaptive polynomial filters. Signal Processing Magazine, IEEE, 8(3):10-26, July 1991.

[41] Michael J. Korenberg. Parallel cascade identification and kernel estimation for nonlinear systems. Annals of Biomedical Engineering, 19(4):429-455, Jul 1991.

[42] Moctar Mossi, Christelle Yemdji, Nicholas Evans, and Christophe Beaugeant. A comparative assessment of noise and non-linear echo effects in acoustic echo cancellation. In ICSP 2010, 4th International Conference on Signal Processing, October 24-28, 2010, Beijing, China, Beijing, CHINA, 102010.

[43] J. S. Garofolo, L. F. Lamel, W. M. Fisher, J. G. Fiscus, D. S. Pallett, and N. L. Dahlgren. DARPA TIMIT acoustic phonetic continuous speech corpus CDROM, 1993.

[44] J.K. Nielsen, J.R. Jensen, S.H. Jensen, and M.G. Christensen. The singleand multichannel audio recordings database (smard). In International Workshop Acoustic Signal Processing, Sep. 2014.

[45] Andrzej B. Dobrucki, Benoit Merit, Valérie Lemarquand, and Guy Lemarquand. Modeling of the nonlinear distortion in electrodynamic loudspeakers caused by the voice-coil inductance. In Société Française d'Acoustique SFA, editor, 10ème Congrès Français d'Acoustique, pages -, Lyon, France, April 2010. 


\section{APENNDICE A - CANCELADORES DE ECO ACÚSTICO}

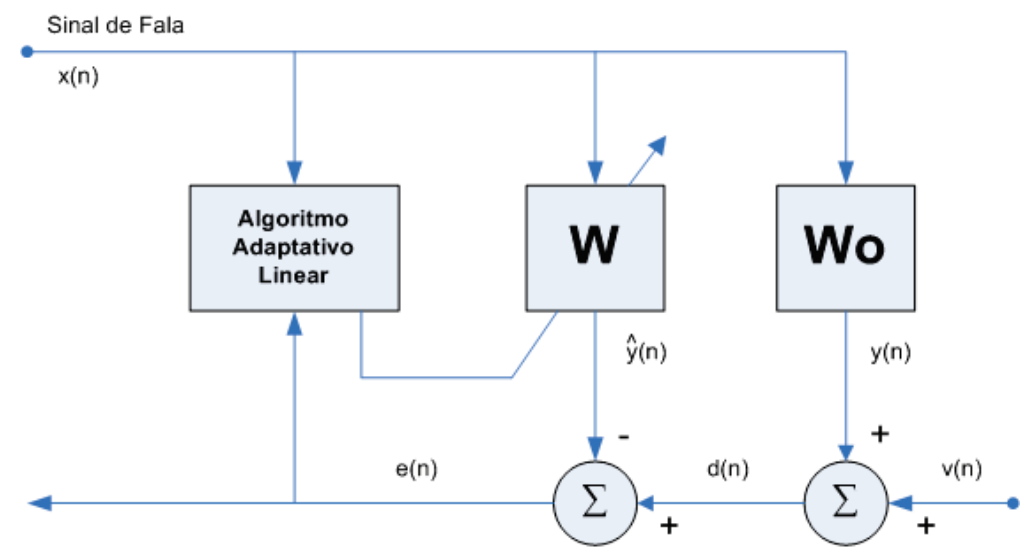

Figura 49: AEC(IL).

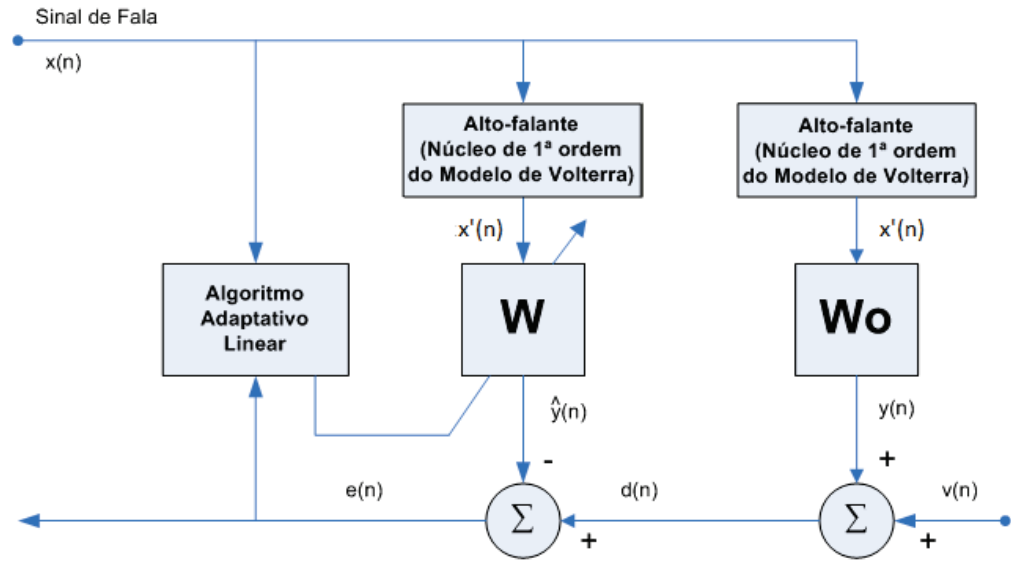

Figura 50: LPAEC(LL). 


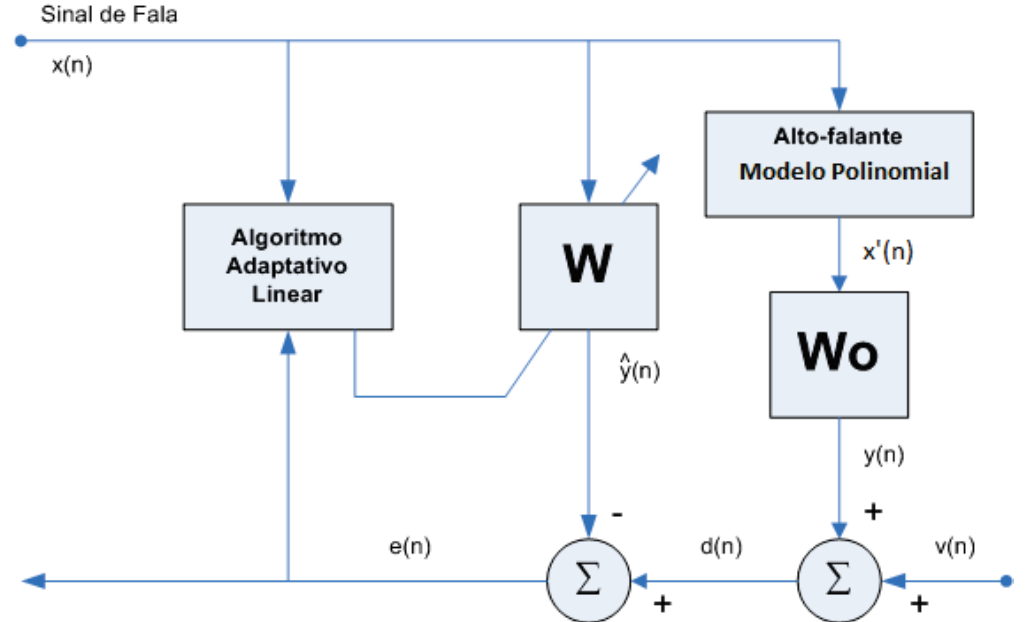

Figura 51: AEC(NLIL).

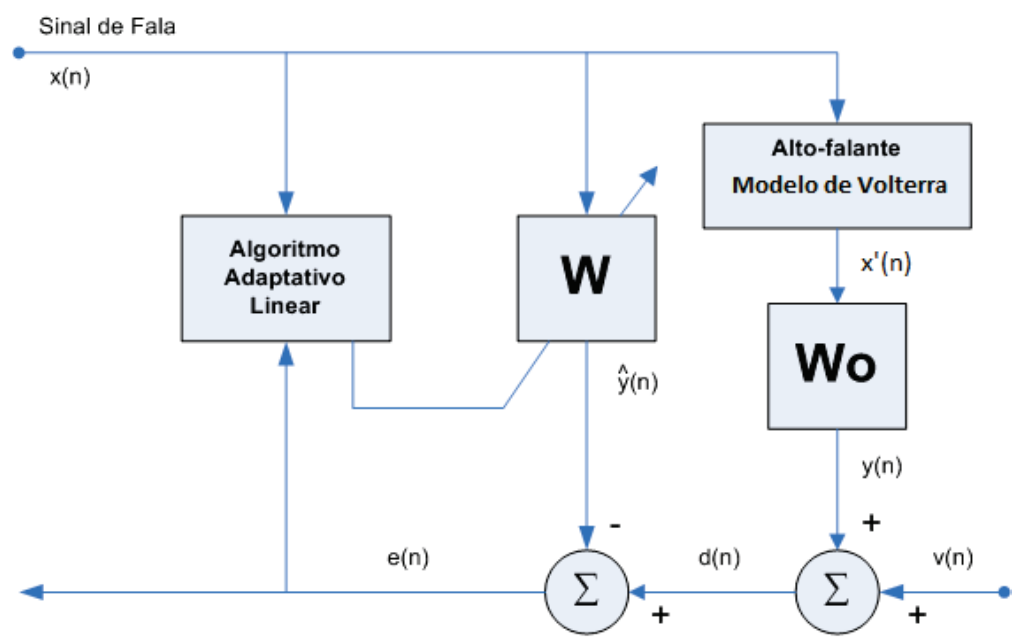

Figura 52: AEC(NLML).

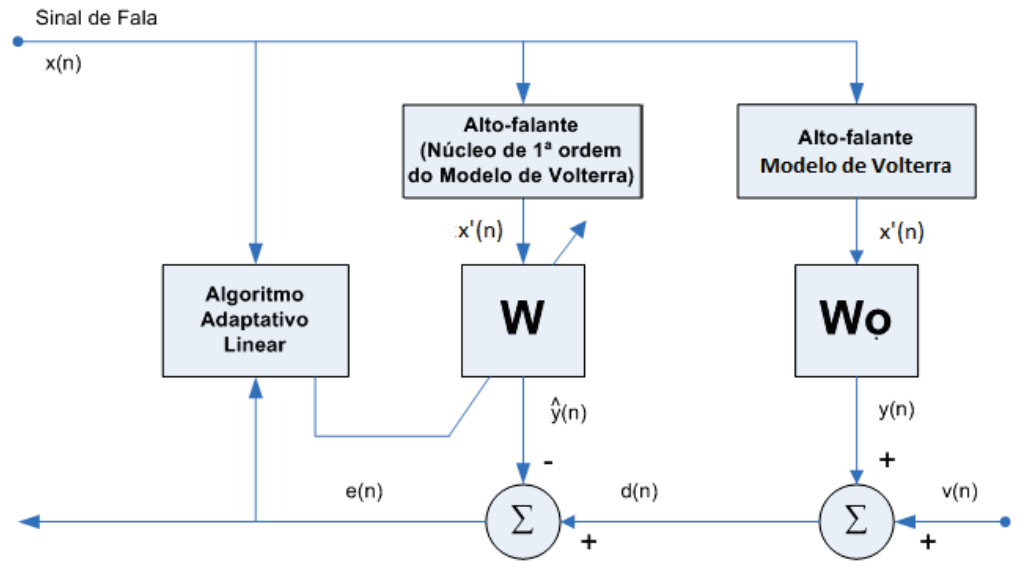

Figura 53: LPAEC(NLML). 


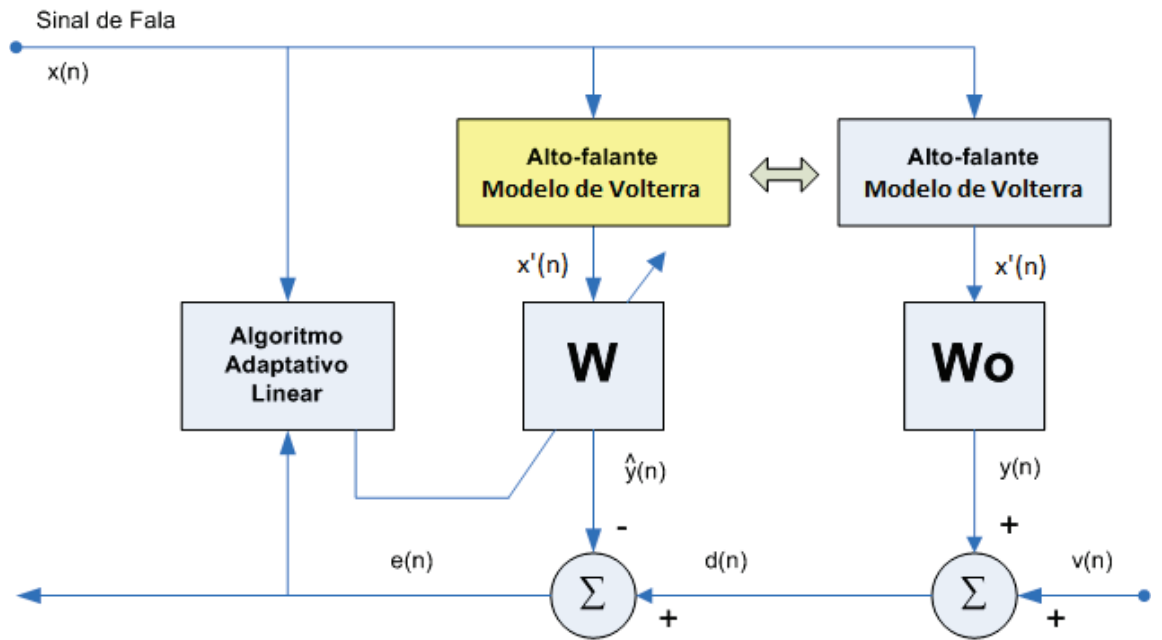

Figura 54: NLPAEC(NLML). 


\section{APÊNDICE B - ALGORITMO LMS}

1 function $[\mathrm{db}, \mathrm{eb}, \mathrm{wb}]=\operatorname{lms}(\mathrm{mu}, \mathrm{N}, \mathrm{M}, \mathrm{x}, \mathrm{d})$

2

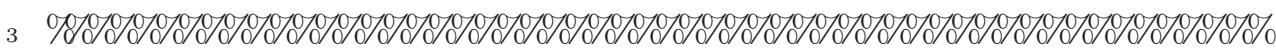

${ }_{4} \% \quad$ Algoritmo LMS $\%$

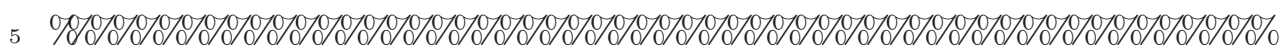

6 \% Chamada: $\%$

$7 \%[\mathrm{db}, \mathrm{eb}, \mathrm{wb}]=\operatorname{lms}($ alpha $, \mathrm{M}, \mathrm{x}, \mathrm{d}) ; \quad \%$

$8 \%$

9 Parametros de entrada: $\%$

${ }_{10} \% \mathrm{mu}=$ passo,$\quad \operatorname{dim} 1 \mathrm{x} 1 \quad \%$

$11 \% \mathrm{~N}=$ numero total de amostras, $\quad \operatorname{dim} 1 \times 1 \quad \%$

$12 \% \mathrm{M}=$ ordem do filtro/bloco, $\quad \operatorname{dim} 1 \times 1 \%$

${ }_{13} \% \mathrm{x}=\operatorname{sinal}$ de entrada, $\quad \operatorname{dim} \mathrm{Nx} 1 \%$

${ }_{14} \% \mathrm{~d}=\operatorname{sinal}$ desejado, $\quad \operatorname{dim} \mathrm{Nx} 1 \%$

$15 \%$

$16 \%$ Parametros de saida: $\%$

${ }_{17} \% \mathrm{db}=$ sinal desejado por bloco, $\quad \operatorname{dim} \mathrm{Nx} 1 \quad \%$

${ }_{18} \%$ eb $=$ erro estimado por bloco, $\quad \operatorname{dim} \mathrm{Nx} 1 \%$

${ }_{19} \% \mathrm{wb}=$ coeficientes do filtro por bloco, $\operatorname{dim} \operatorname{Mx} 1 \%$

${ }_{20} \quad \%\left(\begin{array}{l}0 \\ 0\end{array}\right.$

21

22

${ }_{23} \%$ inicializacao 


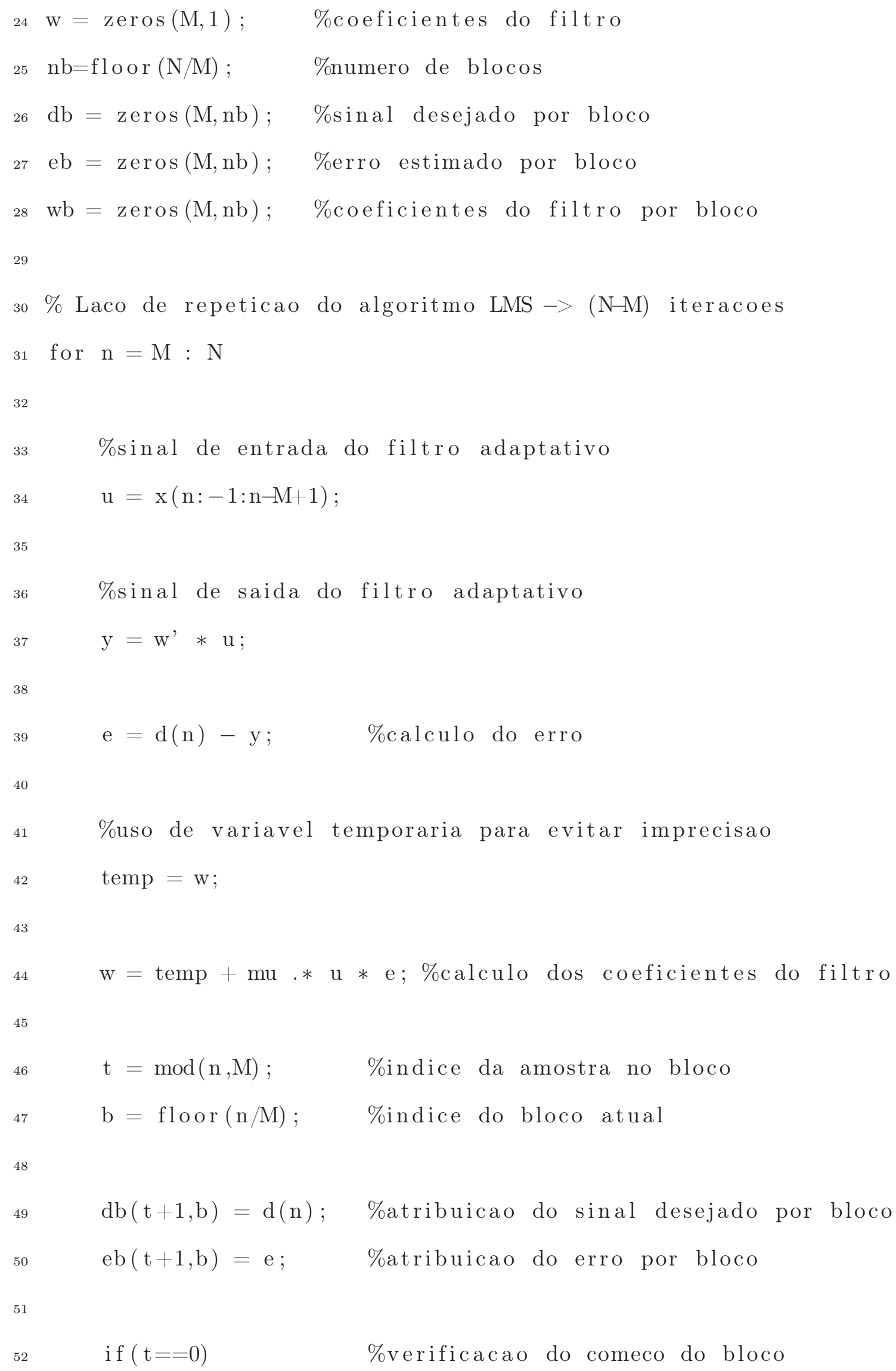


$w b(:, b)=w ; \quad$ \%atribuicao dos coeficientes por bloco

54 end

55 end 


\section{APÊNDICE C - ALGORITMO NLMS}

1 function $[\mathrm{db}, \mathrm{eb}, \mathrm{wb}]=\mathrm{nlms}(\mathrm{mu}, \mathrm{N}, \mathrm{M}, \mathrm{x}, \mathrm{d})$

2

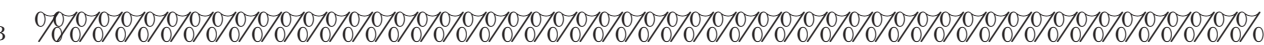

${ }_{4} \% \quad$ Algoritmo NLMS $\%$

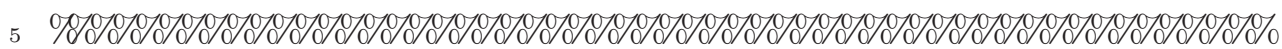

6 \% Chamada: $\%$

$7 \%[\mathrm{db}, \mathrm{eb}, \mathrm{wb}]=\mathrm{nlms}(\mathrm{alpha}, \mathrm{M}, \mathrm{x}, \mathrm{d}) ; \quad \%$

$8 \%$

$9 \%$ Parametros de entrada: $\%$

$10 \% \mathrm{mu}=$ passo, $\quad \operatorname{dim} 1 \times 1 \quad \%$

${ }_{11} \% \mathrm{~N}=$ numero total de amostras, $\quad \operatorname{dim} 1 \times 1 \quad \%$

$12 \% \mathrm{M}=$ ordem do filtro/bloco, $\quad \operatorname{dim} 1 \mathrm{x} 1 \quad \%$

${ }_{13} \% \mathrm{x}=\operatorname{sinal}$ de entrada, $\quad \operatorname{dim} \mathrm{Nx} 1 \%$

${ }_{14} \% \mathrm{~d}=\operatorname{sinal}$ desejado, $\quad \operatorname{dim} \mathrm{Nx} 1 \%$

$15 \%$

$16 \%$ Parametros de saida: $\%$

${ }_{17} \% \mathrm{db}=$ sinal desejado por bloco, $\quad \operatorname{dim} \mathrm{Nx} 1 \quad \%$

${ }_{18} \%$ eb $=$ erro estimado por bloco, $\quad \operatorname{dim} \mathrm{Nx} 1 \quad \%$

${ }_{19} \% \mathrm{wb}=$ coeficientes do filtro por bloco, $\operatorname{dim} \operatorname{Mx} 1 \%$

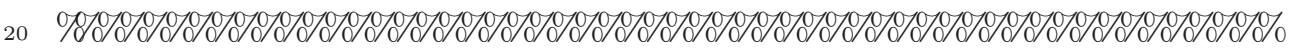

21

${ }_{22} \%$ inicializacao

${ }_{23} \mathrm{w}=\operatorname{zeros}(\mathrm{M}, 1) ; \quad$ \%coeficientes do filtro 


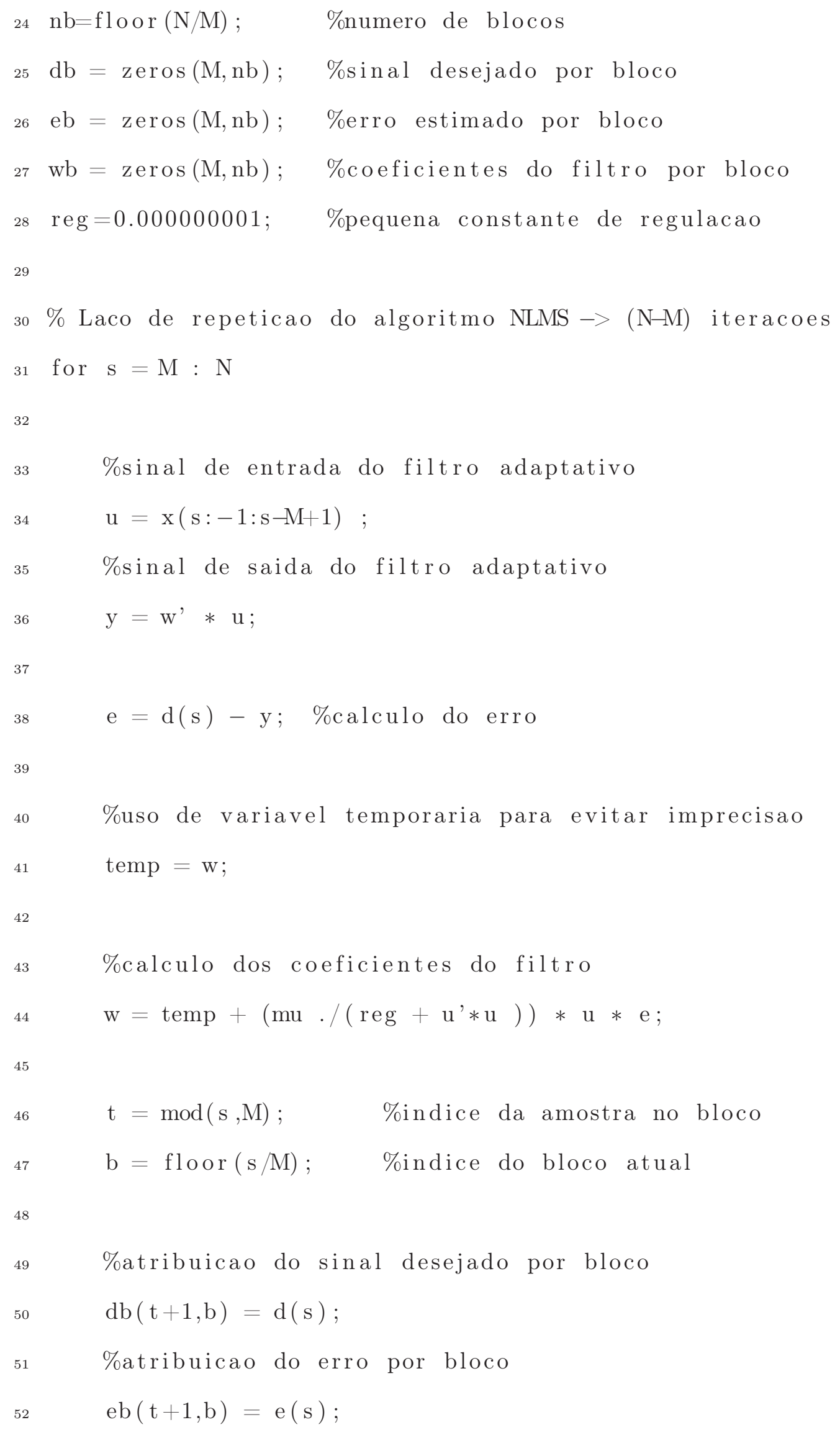


53

54

55

56

57

58

59

\%verificacao do comeco do bloco

if $(\mathrm{t}==0)$

\%atribuicao dos coeficientes por bloco

$\mathrm{wb}(:, \mathrm{b})=\mathrm{w} ;$

end

end 


\section{APÊNDICE D - ALGORITMO FBLMS}

1 function $[\mathrm{db}, \mathrm{eb}, \mathrm{wb}]=$ fblms $(\mathrm{mu}, \mathrm{M}, \mathrm{u}, \mathrm{d}$, gamma $)$

2

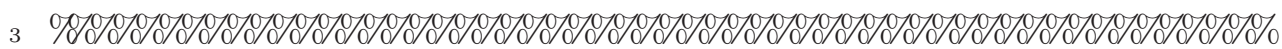

${ }_{4} \% \quad$ Algoritmo FBLMS $\%$

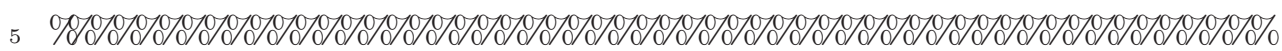

6 \% Chamada: $\%$

$7 \%[\mathrm{db}, \mathrm{eb}, \mathrm{wb}]=\mathrm{fblms}(\mathrm{mu}, \mathrm{M}, \mathrm{u}, \mathrm{d}$, gamma $) ; \quad \%$

$8 \%$

9 Parametros de entrada: $\%$

${ }_{10} \% \mathrm{mu}=$ passo,$\quad \operatorname{dim} 1 \mathrm{x} 1 \quad \%$

$11 \% \mathrm{M}=$ ordem do filtro/bloco, $\quad \operatorname{dim} 1 \times 1 \quad \%$

$12 \% \mathrm{x}=\operatorname{sinal}$ de entrada, $\quad \operatorname{dim} \mathrm{Nx} 1 \quad \%$

${ }_{13} \% \mathrm{~d}=\operatorname{sinal}$ desejado, $\quad \operatorname{dim} \mathrm{Nx} 1 \quad \%$

${ }_{14} \%$ gamma $=$ fator de esquecimento, $\quad \operatorname{dim} 1 \times 1 \quad \%$

$15 \%$

$16 \%$ Parametros de saida: $\%$

${ }_{17} \% \mathrm{db}=$ sinal desejado por bloco, $\quad \operatorname{dim} \mathrm{Nx} 1 \quad \%$

${ }_{18} \%$ eb $=$ erro estimado por bloco, $\quad \operatorname{dim} \mathrm{Nx} 1 \quad \%$

${ }_{19} \% \mathrm{wb}=$ coeficientes do filtro por bloco, $\operatorname{dim} \operatorname{Mx} 1 \quad \%$

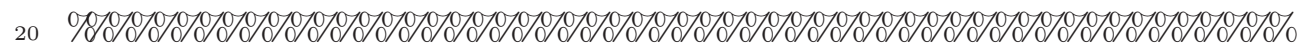

21

$22 \%$ inicializacao

23 W=zeros $(2 * \mathrm{M}, 1) ; \quad$ \%coeficientes do filtro 


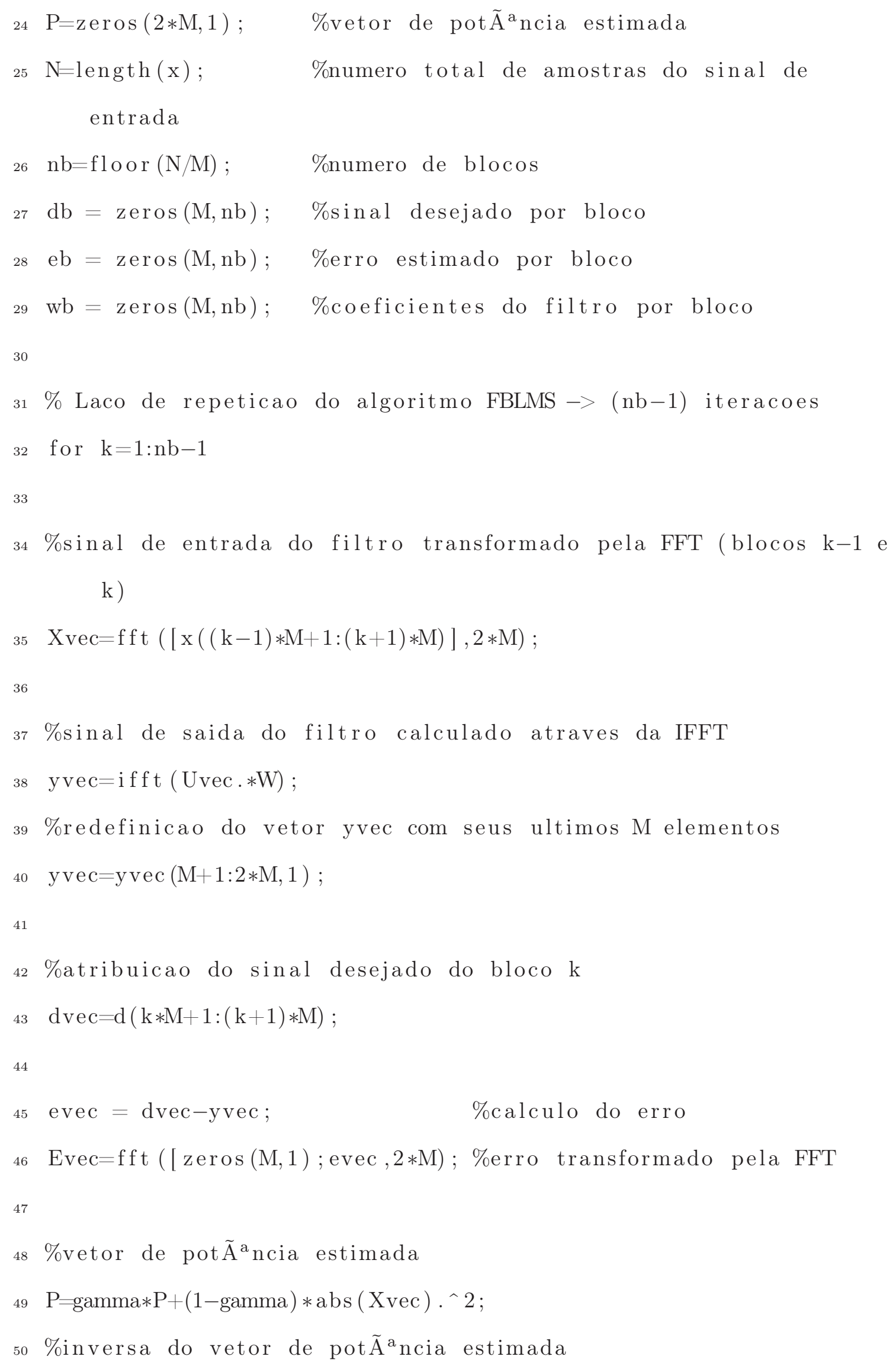


60

${ }_{61}$ \%vetor de erro deve ter valores reais

62 evec=real (evec);

63

$64 \%$ coeficientes do filtro transformados pela IFFT

$65 \mathrm{~W}=\mathrm{ifft}(\mathrm{W})$;

66 \%coeficientes devem ter valores reais

$67 \mathrm{~W}=\mathrm{real}(\mathrm{w}(1: \operatorname{length}(\mathrm{W}) / 2)) ;$

68

69 \%atribuicao do sinal desejado por bloco a variavel de saida

$70 \mathrm{db}(:, \mathrm{k})=\mathrm{dvec} ;$

71 \%atribuicao do erro por bloco a variavel de saida

$72 \mathrm{eb}(:, \mathrm{k})=\mathrm{evec} ;$

73 \%atribuicao dos coeficientes por bloco a variavel de saida

$74 \quad \mathrm{wb}(:, \mathrm{k})=\mathrm{w}$;

75 end 


\section{APÊNDICE E - ALGORITMO APA}

1 function $[\mathrm{db}, \mathrm{eb}, \mathrm{wb}]=\operatorname{apa}(\mathrm{mu}, \mathrm{M}, \mathrm{x}, \mathrm{d})$

2

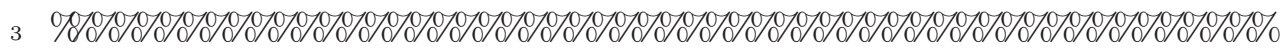

${ }_{4} \% \quad$ Algoritmo APA $\%$

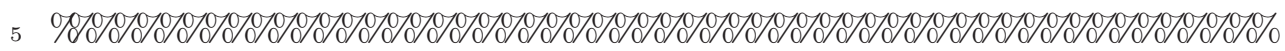

6 \% Chamada: $\%$

${ }_{7} \%[\mathrm{db}, \mathrm{eb}, \mathrm{wb}]=\mathrm{apa}(\mathrm{mu}, \mathrm{M}, \mathrm{x}, \mathrm{d}) ; \quad \%$

$8 \% \%$

9 \% Parametros de entrada: $\%$

${ }_{10} \% \mathrm{mu}=$ passo, $\quad \operatorname{dim} 1 \times 1 \quad \%$

${ }_{11} \% \mathrm{M}=$ ordem do filtro/bloco, $\quad \operatorname{dim} 1 \mathrm{x} 1 \quad \%$

${ }_{12} \% \mathrm{x}=\operatorname{sinal}$ de entrada, $\quad \operatorname{dim} \mathrm{Nx} 1 \quad \%$

${ }_{13} \% \mathrm{~d}=$ sinal desejado, $\quad \operatorname{dim} \mathrm{Nx} 1 \quad \%$

$14 \%$

${ }_{15} \%$ Parametros de saida: $\%$

${ }_{16} \% \mathrm{db}=$ sinal desejado por bloco, $\quad \operatorname{dim} \mathrm{Nx} 1 \quad \%$

${ }_{17} \%$ eb $=$ erro estimado por bloco, $\quad \operatorname{dim} \mathrm{Nx} 1 \quad \%$

${ }_{18} \%$ wb $=$ coeficientes do filtro por bloco, $\operatorname{dim} \operatorname{Mx} 1 \quad \%$

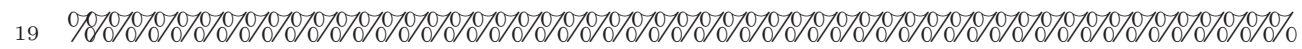

20

${ }_{21} \%$ inicializacao 
$23 \%$ n $\tilde{A}^{\circ}$ mero de amostras de cada vetor de entrada da matrix Xap = M $+1$

${ }_{24} \mathrm{M}=\mathrm{M}-1$;

${ }_{25} \%$ n $\widetilde{A}^{\circ}$ mero de vetores de entrada da matrix Xap $=\mathrm{L}+1$

${ }_{26} \mathrm{~L}=\mathrm{M}$;

${ }_{27} \%$ coeficientes do filtro

$28 \mathrm{~W}=\mathrm{zeros}(\mathrm{L}+1,1)$;

${ }_{29}$ \%calculo do numero total de amostras do sinal de entrada

$30 \mathrm{~N}=\operatorname{length}(\mathrm{x})$;

$31 \%$ matrix do sinal de entrada por bloco $(\mathrm{L}+1 \mathrm{x} \mathrm{M}+1)$

32 Xap=zeros $(\mathrm{L}+1, \mathrm{M}+1)$;

33

$34 \mathrm{nb}=$ floor $(\mathrm{N} /(\mathrm{M}+\mathrm{L}+1)) ; \quad$ \%numero de blocos

${ }_{35} \mathrm{db}=\operatorname{zeros}(\mathrm{L}+1, \mathrm{nb}) ; \quad \%$ sinal desejado por bloco

36 eb $=$ zeros $(\mathrm{L}+1, \mathrm{nb}) ; \quad$ \%erro estimado por bloco

${ }_{37} \mathrm{wb}=\operatorname{zeros}(\mathrm{L}+1, \mathrm{nb}) ; \quad$ \%coeficientes do filtro por bloco

$38 \quad$ zeta $=1 ;$

39 In $=$ eye $(\mathrm{L}+1) ;$

40

${ }_{41} \%$ Laco de repeticao do algoritmo APA $\rightarrow$ nb iteracoes

42 for $\mathrm{k}=\mathrm{M}+\mathrm{L}+1: \mathrm{M}+\mathrm{L}+1: \mathrm{N}$

43

${ }_{44}$ for $\mathrm{li}=0: \mathrm{L}$

${ }_{45} \%$ matrix do sinal de entrada por bloco definido como $\mathrm{L}+1 \mathrm{x} \mathrm{M}+$ 1

${ }_{46} \operatorname{Xap}(\mathrm{li}+1,:)=\mathrm{x}(\mathrm{k}-\mathrm{li}:-1: \mathrm{k}-\mathrm{li}-\mathrm{M})$;

47 end

48

$49 \%$ sinal de saida do filtro adaptativo 


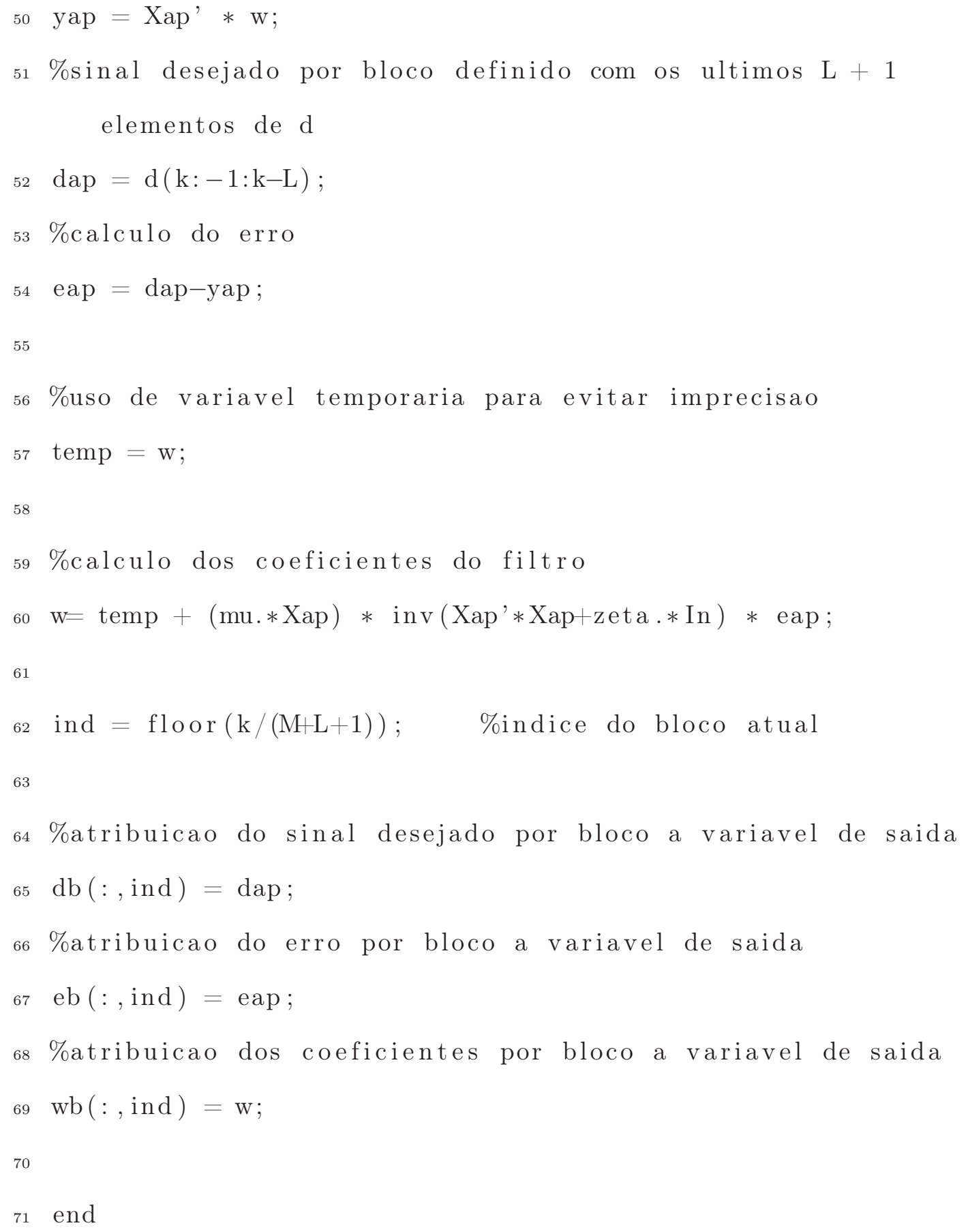




\section{APÊNDICE F - MODELO DE PARÂMETROS PASSIVOS}

Nas seções desse apêndice serão apresentadas breves descrições dos conceitos envolvidos na modelagem do alto-falante.

\section{F.1 Modelo de Parâmetros Passivos}

Como mencionado em [19], sob algumas considerações simplificadoras, modelos parametrizáveis podem ser usados para descrever os efeitos não-lineares dominantes para uma grande variedade de transdutores.

Em um alto-falante eletrodinâmico tanto um campo magnético não homogêneo quanto a rigidez dependente da excursão da bobina de voz representam um comportamento não-linear do transdutor. A terceira causa é a indutância não-linear da bobina de voz, que é frequentemente subestimada. A impedância elétrica da bobina de voz em baixas frequências é considerada geralmente como uma resistência pura, por isso a influência desta indutância é negligenciada [45].

O modelo parametrizável do alto-falante pressupõe a característica de passividade dos seus componentes contituintes, permitindo que o sistema seja descrito como um circuito elétrico passivo. Este, por sua vez, é derivado através de conceitos físicos elementares considerando a energia e a potência das partes elétrica e mecânica do sistema. 
A indutância da bobina de voz depende tanto do deslocamento do diafragma quanto da corrente que flui pela própria bobina de voz. A derivação do modelo proposto por [19] procede da seguinte maneira. Primeiro, alguns conceitos básicos sobre indutâncias não-lineares controladas por corrente são mencionados. Em seguida, uma indutância não-linear dependente do deslocamento do diafragma é investigada e, por meio desse elemento, um transdutor de duas portas é derivado. Por último, o circuito elétrico que representa o modelo é apresentado, assim como suas equações de espaço de estado. Em particular, nenhum detalhe sobre o campo magnético no transdutor é considerado.

\section{F.2 Indutância Não-Linear}

Segundo princípio físico elementar, no caso linear a indutância L é constante e a energia armazenada em função da corrente i é representada pela equação

$$
w(i)=\frac{1}{2} L i^{2}
$$

Uma representação em forma de circuito elétrico da indutância não-linear $L(i)$, dependente da corrente $i$, consiste em um transformador ideal que é terminado com uma indutância linear representada por uma constante positiva $L^{\prime}$, como mostra a Figura 55.

Escolhendo a relação de espiras de acordo com

$$
n(i)=\sqrt{\frac{L(i)}{L^{\prime}}}>0
$$

nota-se que a energia armazenada no circuito é igual ao caso linear, dado por F.1, 


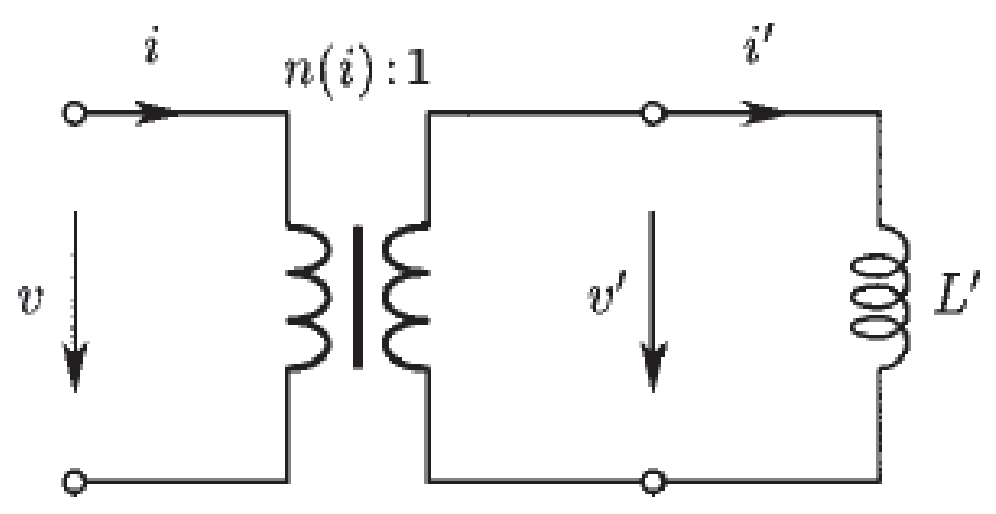

Figura 55: Circuito representando uma indutância não-linear controlada por corrente. Extraído de [19].

como demonstrado a seguir

$$
w(i)=\frac{1}{2} L^{\prime} i^{\prime 2}=\frac{1}{2} L^{\prime}[n(i) i]^{2}=\frac{1}{2} L(i) i^{2} .
$$

\section{F.3 Transdutor de 2-Portas}

As considerações feitas na seção anterior podem ser generalizadas para o caso em que a indutância da bobina não-linear em movimento no entreferro não é apenas dependente da corrente, mas também dependente do deslocamento do diafragma.

De certa forma, a bobina pode ser considerada como um circuito de duas portas sendo acessível através de uma porta elétrica e de uma porta mecânica. Enquanto a corrente $i$ e a tensão $v$ constituem as variáveis na porta elétrica, a velocidade $\dot{x}=\frac{d x}{d t}$ e a força $f$ representam as quantidades correspondentes na porta mecânica.

Para derivar as relações fundamentais entre as variáveis mencionadas, consi- 


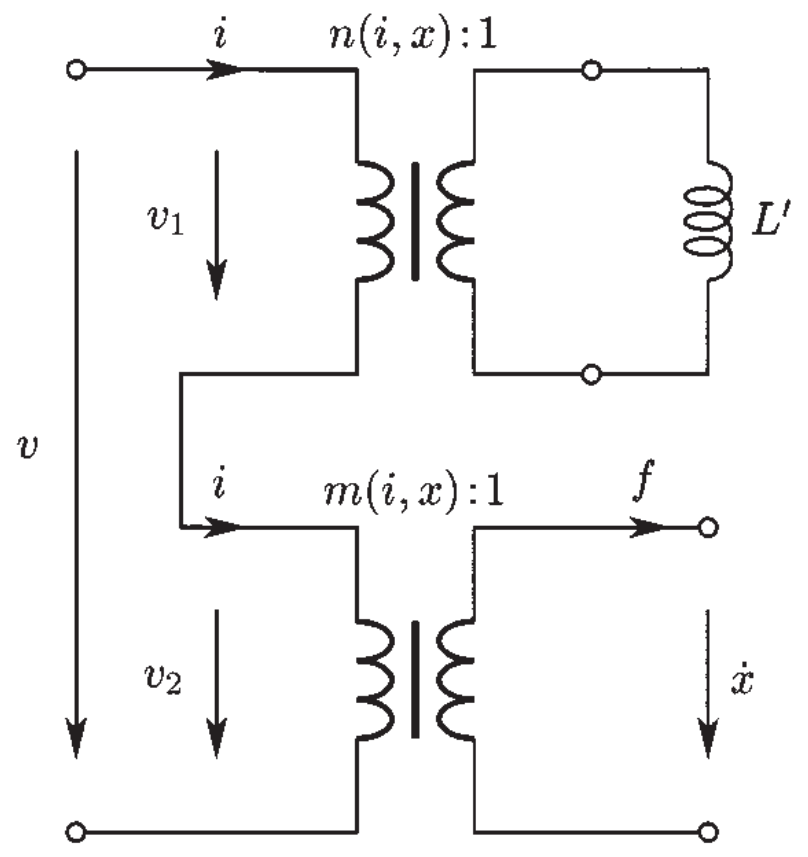

Figura 56: Circuito referente a bobina em movimento no campo magnético. Extraído de [19].

deramos a energia armazenada na bobina em movimento

$$
w(i, x)=\frac{1}{2} L(i, x) i^{2}=\frac{1}{2} L^{\prime}[n(i, x) i]^{2} .
$$

Por motivo de simplificação, se admitirmos que a indutância não depende da corrente $i$, então $L(i, x)=L(x)$, o que resulta em relações de espiras independentes de $i$ que podem ser expressas por

$$
n(i, x)=\sqrt{\frac{L(x)}{L^{\prime}}}
$$

e

$$
m(i, x)=a(x)+\frac{i}{2} \frac{\mathrm{d} L(x)}{\mathrm{d} x}
$$

O princípio de conservação de energia implica que o incremento da energia armazenada é igual à soma dos incrementos elétricos e mecânicos da energia fornecida ao sistema. Analisando mais detalhadamente esse incremento de energia 
em termos do fluxo magnético $\phi$ e da força $f$, que devem ser consideradas como funções da corrente $i$ e do deslocamento do diafragma $x$, chega-se às equações de tensão do circuito. As deduções matemáticas envolvidas nessa análise fogem ao escopo do presente trabalho e podem ser inspecionadas em [19].

Assim, pode-se representar a tensão $v$ indicada na Figura 56 pela equação:

$$
v=L(i, x) \frac{\mathrm{d} i}{\mathrm{~d} t}+\frac{i}{2} \frac{\partial L(i, x)}{\partial i} \frac{\mathrm{d} i}{\mathrm{~d} t}+\frac{i}{2} \frac{\partial L(i, x)}{\partial x} \frac{\mathrm{d} x}{\mathrm{~d} t}+m(i, x) \frac{\mathrm{d} x}{\mathrm{~d} t} .
$$

Os três primeiros termos da direita na equação (F.7) podem ser agrupados para representar a tensão causada pela alteração da energia armazenada, correspondendo à tensão $v_{1}$ na Figura 57. Enquanto, o último termo da direita da equação (F.7) corresponde à tensão $v_{2}$ na Figura 57, já que o mesmo pode ser visto como a tensão gerada pelo movimento da bobina causado pela energia armazenada que se conserva.

$$
\begin{gathered}
v_{1}=n(i, x) L^{\prime} \frac{\mathrm{d} n(i, x) i}{\mathrm{~d} t}=L(i, x) \frac{\mathrm{d} i}{\mathrm{~d} t}+\frac{i}{2} \frac{\mathrm{d} L(i, x)}{\mathrm{d} x} \frac{\mathrm{d} x}{\mathrm{~d} t} \\
v_{2}=m(i, x) \frac{\mathrm{d} x}{\mathrm{~d} t}=a(x) \frac{\mathrm{d} x}{\mathrm{~d} t}+\frac{i}{2} \frac{\mathrm{d} L(i, x)}{\mathrm{d} x} \frac{\mathrm{d} x}{\mathrm{~d} t} .
\end{gathered}
$$

\section{F.4 Circuito Elétrico}

Para complementar o circuito elétrico do modelo conecta-se uma fonte ideal de tensão e um resistor à porta elétrica. O resistor deve representar os efeitos da resistência de saída do amplificador de potência e da resistência da bobina. Na outra porta, conecta-se um circuito ressonante paralelo não-linear amortecido, conforme representado na Figura 57, representando um sistema de massa-mola amortecida e simulando, em particular, o diafragma excitado e a sua suspensão. 
O movimento da massa e o coeficiente de atrito são assumidos como constantes; consequentemente, eles são representados por elementos de circuito linear, isto é, por uma capacitância e uma condutância, respectivamente [19].

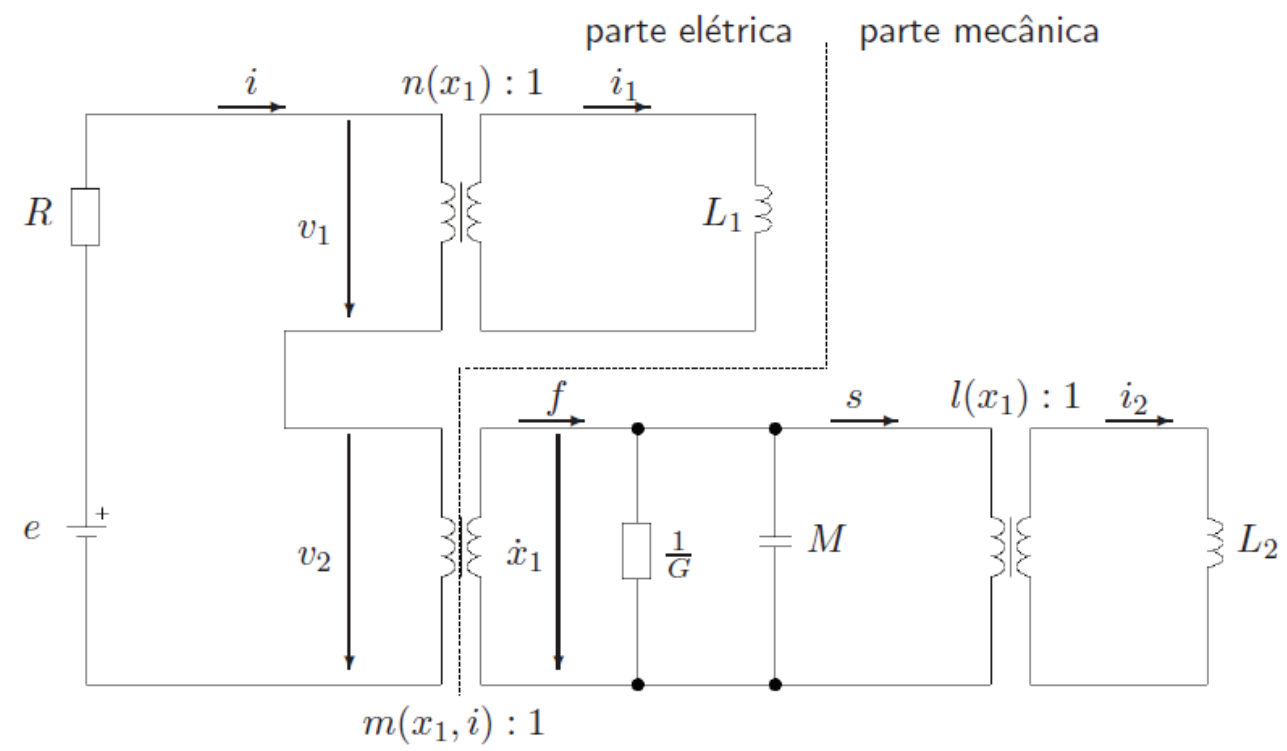

Figura 57: Modelo de circuito elétrico de um alto-falante eletrodinâmico. Extraído de [19].

Analisando o circuito da Figura 57 nota-se que a equação da malha da parte elétrica à esquerda pode ser expressa por

$$
e=R i+v_{1}+v_{2} .
$$

Usando as equações (F.8) e (F.9), temos:

$$
e=R i+L(i, x) \frac{\mathrm{d} i}{\mathrm{~d} t}+i \frac{\mathrm{d} L(i, x)}{\mathrm{d} x} \frac{\mathrm{d} x}{\mathrm{~d} t}+a(x) \frac{\mathrm{d} x}{\mathrm{~d} t} .
$$




\section{F.5 Equações de espaço de estados}

Com o objetivo de obter as equações de espaço de estado do modelo define-se o vetor de estados como

$$
\boldsymbol{x}=\left[\begin{array}{l}
x \\
\dot{x} \\
i
\end{array}\right]=\left[\begin{array}{l}
x_{1} \\
x_{2} \\
x_{3}
\end{array}\right] .
$$

Por inspeção da equação (57), a equação diferencial de $x_{1}$ é dada por

$$
\dot{x_{1}}=x_{2} \text {. }
$$

Para obter a equação diferencial de $x_{2}$ é necessário analisar a parte mecânica do circuito elétrico da Figura 57 aplicando a lei de Newton como segue:

$$
M \ddot{x}+G \dot{x}=f-s,
$$

onde $f$ denota a força resultante da bobina em movimento e $s$ representa a força de reação da suspensão, que atua como uma mola, $M$ é a massa do diafragma e a condutância $G$ representa o coeficiente de atrito da massa em movimento.

A força de reação da suspensão é modelada considerando que sua rigidez depende do deslocamento do diafragma como segue:

$$
s\left(x_{1}\right)=k\left(x_{1}\right) x_{1},
$$

onde $k\left(x_{1}\right)$ é a função de rigidez.

Verifica-se através de (F.12) que $\dot{x_{2}}=\ddot{x_{1}}$, logo se considerarmos $\ddot{x_{1}}$ segundo a lei de Newton expressa por (F.14) e a força de rigidez definida por (F.15), 
podemos expressar a equação diferencial de $x_{2}$ como:

$$
\dot{x_{2}}=\frac{1}{M}\left(f-G x_{2}-k\left(x_{1}\right) x_{1}\right) .
$$

Por inspeção do circuito elétrico da Figura 57, pode-se representar a força $f$ pela expressão:

$$
f=m(i, x) i
$$

Analisando (F.12), observa-se que $x=x_{1}$ e $i=x_{3}$, logo:

$$
f=m\left(x_{3}, x_{1}\right) x_{3}
$$

E recorrendo à equação (F.6), tem-se

$$
f=a\left(x_{1}\right) x_{3}+\frac{x_{3}^{2}}{2} \frac{\mathrm{d} L\left(x_{1}\right)}{\mathrm{d} x_{1}} .
$$

Assim, equação diferencial de $x_{2}$ é dada por

$$
\dot{x_{2}}=\frac{1}{M}\left\{a\left(x_{1}\right) x_{3}+\frac{x_{3}^{2}}{2} L^{\prime}\left(x_{1}\right)-k\left(x_{1}\right) x_{1}-G x_{2}\right\} .
$$

A equação diferencial de $x_{3}$ é obtida diretamente da expressão (F.11), isto é

$$
\dot{x_{3}}=\frac{1}{L\left(x_{1}\right)}\left\{e-R x_{3}-L^{\prime}\left(x_{1}\right) x_{2} x_{3}-a\left(x_{1}\right) x_{2}\right\} .
$$

Finalmente, a representação do modelo em equações de espaço de estados é 
dada por

$$
\begin{gathered}
\dot{x}=\left[\begin{array}{c}
x_{2} \\
\frac{1}{M}\left\{a\left(x_{1}\right) x_{3}+\frac{x_{3}^{2}}{2} L^{\prime}\left(x_{1}\right)-k\left(x_{1}\right) x_{1}-G x_{2}\right\} \\
-\frac{1}{L\left(x_{1}\right)}\left\{R x_{3}+L^{\prime}\left(x_{1}\right) x_{2} x_{3}+a\left(x_{1}\right) x_{2}\right\}
\end{array}\right]+\left[\begin{array}{c}
0 \\
0 \\
\frac{1}{L\left(x_{1}\right)}
\end{array}\right] e \\
y=\left[\begin{array}{lll}
1 & 0 & 0
\end{array}\right] x .
\end{gathered}
$$

As funções $a\left(x_{1}\right), k\left(x_{1}\right)$ e $L\left(x_{1}\right)$ que modelam o comportamento do fator de força, da rigidez da suspensão do diafragma e da indutância não-linear da bobina de voz são descritas abaixo, conforme [19].

$$
\begin{gathered}
a\left(x_{1}\right)=\frac{a_{0}}{1+a_{1}\left(x_{1}-x_{a}\right)^{2}} \\
k\left(x_{1}\right)=k_{0}+k_{1}\left(x_{1}-x_{k}\right)^{2} \\
L\left(x_{1}\right)=l_{0}+l_{1}\left(1-\frac{x_{1}-x_{l}}{l_{2}+\left\|x_{1}-x_{l}\right\|}\right) .
\end{gathered}
$$

As equações aproximadas de $a\left(x_{1}\right)$ e $L\left(x_{1}\right)$ são derivadas em [19] através da inspeção do comportamento dos componentes do alto-falante eletrodinâmico nos limites de deslocamento do diafragma. Por sua vez, a equação aproximada de $k\left(x_{1}\right)$ é originalmente proposta em [35]. 


\section{APÊNDICE G - ALGORITMO BILINEAR ESPARSO}

1 function $[\mathrm{x}]=\mathrm{af}_{\text {_ bilinear }}(\mathrm{T}, \mathrm{N}, \mathrm{e})$

2

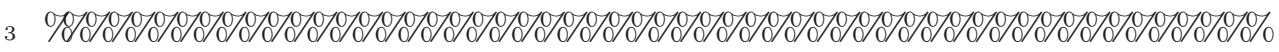

${ }_{4} \% \quad$ Algoritmo Bilinear Esparso $\%$

5

6 \% Chamada: $\%$

$7 \%[\mathrm{x}]=$ af_bilinear $(\mathrm{tspan}, \mathrm{N}, \mathrm{e}) \quad \%$

$8 \%$

9 \% Parametros de entrada: $\%$

$10 \% \mathrm{~T}=$ intervalo de tempo de amostragem, $\operatorname{dim} 1 \mathrm{x} 1 \%$

$11 \% \mathrm{~N}=$ numero total de amostras, $\quad \operatorname{dim} 1 \times 1 \quad \%$

$12 \%$ e $=$ sinal de entrada, $\quad \operatorname{dim} \mathrm{Nx} 1 \quad \%$

${ }_{13} \% \quad \%$

$14 \%$ Parametros de saida: $\quad \%$

${ }_{15} \% \mathrm{x}=$ sinal de saida do sistema bilinear $\operatorname{dim} \mathrm{Nx} 1 \quad \%$

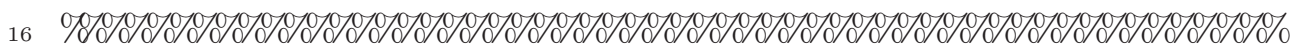

17

$18 \%$ Arquivo com valores pre-calculados $\operatorname{arq}=$ 'mCB_AF_P3_Bilinear';

\% Carrega F, G e b pre-calculados

21 load $(\operatorname{arq})$; 
$\%$ Determina a matriz esparsa Fs

$[\operatorname{rowF}, \operatorname{colF}, \mathrm{Fs}]=$ find $(\mathrm{F})$;

$\%$ Determina a matriz esparsa Gs

$[$ rowG $, \operatorname{colG}, \mathrm{Gs}]=$ find $(\mathrm{G})$;

\% Captura comprimento de F (matriz quadrada)

$\operatorname{dim} F=\operatorname{size}(F, 1) ;$

\% Captura comprimento de G (matriz quadrada)

$\operatorname{dim} G=\operatorname{size}(\mathrm{G}, 1) ;$

$\%$ Inicializa Fr com zeros

$\operatorname{Fr}=\operatorname{zeros}(\operatorname{dimF}, 1) ;$

$\%$ Inicializa Gr com zeros

$\mathrm{Gr}=\operatorname{zeros}(\operatorname{dimG}, 1)$;

$\%$ vetor de estado inicial

$\%$ [deslocamento, velocidade, corrente ,...]

$\mathrm{x} 0=$ zeros(length $(\mathrm{b}), 1) ; \%$ inicializacao com zeros

$\%$ vetor de sa Âda com valores temporarios

$\mathrm{xk}=\operatorname{zeros}(\mathrm{N}, 1) ; \quad \%$ inicializacao com zeros

for $j=1: N$

$$
\begin{aligned}
& \text { \% calcula o vetor } \mathrm{Fr} \\
& \text { for } \mathrm{i}=1: \operatorname{dim} F-1 \\
& \qquad \begin{aligned}
\text { for } \mathrm{k}=\operatorname{rowF}(\mathrm{i}): \operatorname{rowF}(\mathrm{i}+1) \\
\\
\operatorname{Fr}(\mathrm{i})=\operatorname{Fr}(\mathrm{i})+\mathrm{Fs}(\mathrm{k}) * \mathrm{x} 0(\operatorname{colF}(\mathrm{k})) ;
\end{aligned}
\end{aligned}
$$


72 end

end

end

$\%$ calcula o vetor Gr

for $i=1$ :dim $G-1$

for $k=\operatorname{row} G(i): \operatorname{row} G(i+1)$

$\operatorname{Gr}(\mathrm{i})=\operatorname{Gr}(\mathrm{i})+\operatorname{Gs}(\mathrm{k}) * \mathrm{x} 0(\operatorname{colG}(\mathrm{k}))$;

end

end

\% calcula a saida do sistema bilinear para $e(j)$

$\mathrm{x}=\mathrm{x} 0+(\mathrm{T} \cdot *(\mathrm{Fr}+\mathrm{Gr} \cdot * \mathrm{e}(\mathrm{j})))+(\mathrm{T} \cdot *(\mathrm{~b} \cdot * \mathrm{e}(\mathrm{j}))) ;$

$x k(j)=x(1) ; \quad \%$ atualiza o vetor temporario

$\mathrm{x} 0=\mathrm{x} ; \quad \%$ atualiza $\quad$ vetor de estados

$\log =\left[\operatorname{xk}\left({ }^{\prime} \operatorname{num} 2 \operatorname{str}(j)^{\prime}\right)={ }^{\prime} \operatorname{num} 2 \operatorname{str}(\operatorname{xk}(j))\right]$;

disp $(\log ) ; \% \log$ dos valores de saida na tela

end

$\mathrm{x}=\mathrm{xk} ; \quad \%$ atualiza vetor de saida 